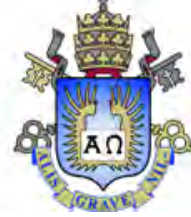

Pedro Tovar Braga

The role of Rayleigh backscattering of coherent light in the mode dynamics of random DFB

fiber lasers

Tese de Doutorado

Thesis presented to the Programa de Pós-Graduação em Engenharia Elétrica of PUC-Rio in partial fulfillment of the requirements for the degree of Doutor em Engenharia Elétrica.

Advisor: Prof. Jean Pierre von der Weid 


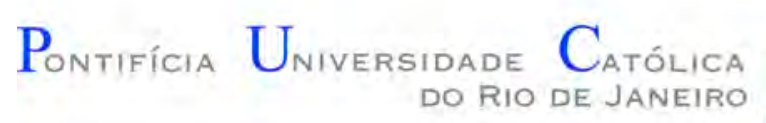

Pedro Tovar Braga

\section{The role of Rayleigh backscattering of coherent light in the mode dynamics of random DFB \\ fiber lasers}

Thesis presented to the Programa de Pós-graduação em Engenharia Elétrica da PUC-Rio in partial fulfillment of the requirements for the degree of Doutor em Engenharia Elétrica. Approved by the Examination Committee:

Prof. Jean Pierre von der Weid

Advisor

Centro de Estudos em Telecomunicações - PUC-Rio

Prof. Walter Margulis

Research Institutes of Sweden - RISE

Prof. Anderson Stevens Leonidas Gomes Universidade Federal de Pernambuco - UFPE

Prof. Ricardo Marques Ribeiro Universidade Federal Fluminense - UFF

Prof. Guilherme Penello Temporão

Centro de Estudos em Telecomunicações - PUC-Rio 
All rights reserved.

\section{Pedro Tovar Braga}

Graduated in Electrical Engineering at PUC-Rio, with emphasis in Telecommunications. Obtained his Master of Science title at the Center for Telecommunication Studies at PUC-Rio, CETUC, focusing on the field of MicrowavePhotonics. In late 2018 he started working with random distributed feedback fiber lasers. He has presented his works in international conferences in the United States and Europe, and has published over twenty papers.

Bibliographic data

Tovar Braga, Pedro

The role of Rayleigh backscattering of coherent light in the mode dynamics of random DFB fiber lasers / Pedro Tovar Braga; advisor: Jean Pierre von der Weid. - 2021.

140 f: il. color. ; $30 \mathrm{~cm}$

Tese (doutorado) - Pontifícia Universidade Católica do Rio de Janeiro, Departamento de Engenharia Elétrica, 2021.

Bibliography included

1. Engenharia Elétrica - Teses. 2. Lasers aleatórios à fibra. 3. Feedback distribuído. 4. Espalhamento Rayleigh. 5. Fibras ópticas. 6. Amplificadores ópticos a semicondutor. I. von der Weid, Jean Pierre. II. Pontifícia Universidade Católica do Rio de Janeiro. Departamento de Engenharia Elétrica. III. Título. 


\section{Acknowledgments}

I would like to thank my advisor Jean Pierre von der Weid, whose teaching capabilities go far beyond optics and photonics. I learned to be curious about every tiny detail, which not often, but occasionally, hides incredible answers, revealed only to those who persist in being curious.

To all my colleagues and friends from the photonics laboratory at PUCRio, who were always there to help and happy to discuss about all the interesting phenomena we witnessed over the years.

To my wife, who enlightens my days with joy and love, being the best life-partner any man could ask for.

To my mother, for her unlimited love and support, and for encouraging me along each and every step of the way.

To my brother. My uttermost example of integrity, who has always chosen the path made of principles, that leads to character.

Without them, this work would not have been possible.

To all my family and friends, who directly or indirectly contributed to this work.

This study was financed in part by the Coordenação de Aperfeiçoamento de Pessoal de Nível Superior - Brasil (CAPES) - Finance Code 001. And I would also like to thank the Brazilian agency CNPq for the financial support. 


\section{Abstract}

Tovar Braga, Pedro; von der Weid, Jean Pierre (Advisor). The role of Rayleigh backscattering of coherent light in the mode dynamics of random DFB fiber lasers. Rio de Janeiro, 2021. 140p. Tese de Doutorado - Departamento de Engenharia Elétrica, Pontifícia Universidade Católica do Rio de Janeiro.

In this thesis, it is provided a thorough analysis of mode dynamics of random distributed feedback fiber lasers. A rigorous investigation is proposed for the feedback mechanism, Rayleigh backscattering, which plays a key role in laser action. Based on the intermediate range order of silica glasses, and on residual stress of optical fibers, a theoretical model was built to predict intensity fluctuations of Rayleigh backscattered coherent light. Model predictions were compared to experimental results, strongly supporting the conclusion that Rayleigh backscattering in single mode fibers is an ergodic process exhibiting ergodicity in the time-frequency sense so that the model can be used to predict the statistical behavior of backscattered intensity fading. The model was used to explain laser action in a novel configuration of random fiber laser, with a semiconductor optical amplifier (SOA) employed as the gain medium. It is here demonstrated that single-mode operation is only possible in pulsed regime at SOA driving currents close to the threshold, whereas multimode regime dominates under higher currents. Experimental results indicate that the mode power is limited by mode competition, which is observed under high SOA currents. Pulsed regime is shown to be due to randomly driven Q-switching induced by a scintillation effect in the Rayleigh backscattered light, which effectively translates as a time-varying cavity loss. Mode lifetimes of $\sim 1 \mathrm{~ms}$ and narrow linewidths ranging from 4 to $7 \mathrm{kHz}$ were experimentally obtained. Brillouin-based random fiber lasers were also analyzed, showing similar mode dynamics, but due to the much narrower gain spectral width, mode competition did not limit the mode power, which was rather limited by the second order Brillouin-Stokes light. Last, intra-cavity phase-modulation experiments showed that laser action can be efficiently controlled by breaking either phase or gain conditions.

\section{Keywords}

Random fiber lasers; Distributed feedback; Rayleigh scattering; Optical fibers; Semiconductor optical amplifie. 


\section{Resumo}

Tovar Braga, Pedro; von der Weid, Jean Pierre. O papel do retro espalhamento Rayleigh de luz coerente na dinâmica dos modos de lasers aleatórios DFB. Rio de Janeiro, 2021. 140p. Tese de Doutorado - Departamento de Engenharia Elétrica, Pontifícia Universidade Católica do Rio de Janeiro.

Nessa tese é analisada a dinâmica dos modos de lasers aleatórios à fibra com feedback distribuído. Um rigoroso estudo é feito sobre o mecanismo de feedback distribuído, i.e., o espalhamento Rayleigh, que é um dos principais elementos contribuindo para emissão laser. É desenvolvido um modelo teórico para previsão do retro espalhamento Rayleigh de luz coerente, o qual é baseado na ordem de médio alcance da sílica, e na tensão residual de fibras ópticas. As previsões do modelo são comparadas com resultados experimentais, levando a conclusão de que o retro espalhamento Rayleigh em fibras ópticas é um processo ergódigo nos domínios do tempo e da frequência óptica. O modelo mostrou-se eficaz na previsão das flutuações de intensidade do retro espalhamento Rayleigh de luz coerente, e foi utilizado para explicar a emissão de luz laser numa nova configuração de laser aleatório à fibra, que faz uso de um amplificador óptico a semicondutor (SOA) como meio de ganho. É demostrado que operação monomodo só é possível em emissão pulsada e com bombeio próximo ao limiar laser, enquanto operação multimodo é dominante para bombeio acima do limiar. Resultados experimentais indicam que potência dos modos é limitada pela competição entre modos por ganho. A operação em regime pulsado é atribuída a um efeito de cintilação da luz Rayleigh retro espalhada, que equivale a um fenômeno de chaveamento de perdas (Q-switching) aleatório na cavidade. O tempo de vida dos modos é $\sim 1 \mathrm{~ms}$ e larguras de linha estreitas, no intervalo entre 4 e $7 \mathrm{kHz}$, foram obtidas experimentalmente. Lasers aleatórios à fibra com ganho Brillouin também foram analisados, mostrando semelhante dinâmica dos modos. Entretanto, por conta da estreita banda espectral do ganho Brillouin a competição entre modos não limitou a potência dos modos, a qual foi observada ser limitada por luz Stokes proveniente do espalhamento Brillouin de segunda ordem. Por último, experimentos com modulação de fase intra-cavidade foram avaliados, e mostraram que emissão laser pode ser controlada a partir da supressão da condição de fase ou ganho.

\section{Palavras-chave}

Lasers aleatórios à fibra; Feedback distribuído; Espalhamento Rayleigh; Fibras ópticas; Amplificadores ópticos a semicondutor. 


\section{Table of contents}

1 Introduction $\quad 18$

$\begin{array}{lll}1.1 & \text { Motivation and background } & 19\end{array}$

$\begin{array}{lll}1.2 & \text { Objectives } & 22\end{array}$

1.3 Historical overview 23

1.4 Relevance 25

$\begin{array}{ll}1.5 & \text { Thesis contributions } \\ 1.6 & 25\end{array}$

1.6 Thesis outline 28

$2 \quad$ Laser background $\quad 30$

2.1 Laser physics 30

2.1.1 Light amplification 31

$\begin{array}{lll}2.1 .2 & \text { Optical resonator } & 35\end{array}$

$\begin{array}{lll}2.1 .3 & \text { Laser action } & 38\end{array}$

2.2 Semiconductor optoelectronics 40

2.2.1 Semiconductor optical amplifier 42

$3 \quad$ Rayleigh scattering $\quad 46$

3.1 Light scattering phenomena $\quad 46$

$\begin{array}{ll}3.2 & \text { Rayleigh backscattering in optical fibers }\end{array}$

3.2.1 Review of Rayleigh backscattering models 48

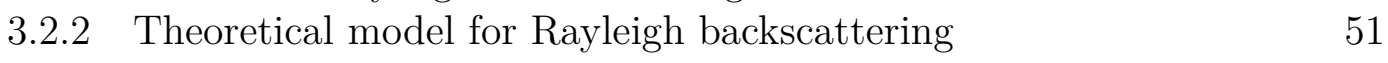

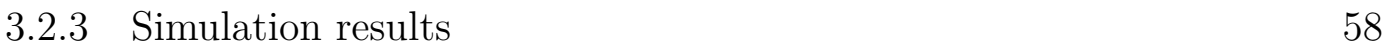

3.3 Ergodicity of Rayleigh backscattering 66

3.4 Experimental results and ergodicity validation 69

4 Random DFB fiber lasers $\quad \mathbf{7 6}$

$\begin{array}{lll}4.1 & \text { Introduction to random lasers } & 76\end{array}$

$\begin{array}{lll}4.2 & \text { Review of random DFB fiber lasers } & 79\end{array}$

$\begin{array}{lll}4.2 .1 & \text { Theoretical background } & 79\end{array}$

4.2.2 Spectral and temporal dynamics 82

4.2.3 Other gain mechanisms 83

4.3 Applications of random lasers 86

$5 \quad$ SOA-based random DFB fiber laser $\quad 88$

$\begin{array}{lll}5.1 & \text { Theoretical analysis } & 88\end{array}$

$\begin{array}{lll}5.2 & \text { Simulation predictions } & 89\end{array}$

$\begin{array}{ll}5.3 \text { Experimental setup } & 94\end{array}$

$\begin{array}{lll}5.4 & \text { Results and discussions } & 95\end{array}$

$\begin{array}{lll}5.4 .1 & \text { Output power } & 95\end{array}$

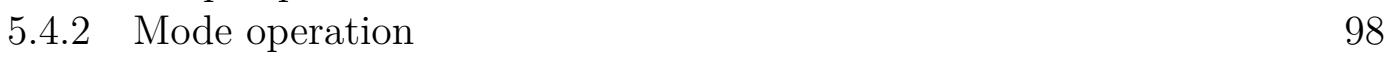

$\begin{array}{lll}\text { 5.4.3 Spectral analysis } & 98\end{array}$

6 Brillouin-based random DFB fiber laser 102

$\begin{array}{lll}6.1 & \text { Introduction } & 102\end{array}$ 
$\begin{array}{lll}6.2 & \text { Experimental setup } & 103\end{array}$

$\begin{array}{lll}6.3 & \text { Results and discussions } & 104\end{array}$

$\begin{array}{lll}7 & \text { Breaking phase and gain conditions } & 111\end{array}$

$\begin{array}{lll}7.1 & \text { Introduction } & 111\end{array}$

$\begin{array}{lll}7.2 & \text { Experimental setup } & 113\end{array}$

$\begin{array}{lll}7.3 & \text { Theoretical analysis } & 114\end{array}$

$\begin{array}{lll}7.4 & \text { Results and discussions } & 119\end{array}$

$8 \quad$ Summary and future works $\quad 124$

$\begin{array}{lll}8.1 & \text { Summary } & 124\end{array}$

$\begin{array}{ll}8.2 \text { Future works } & 126\end{array}$

$\begin{array}{ll}\text { Bibliography } & 127\end{array}$ 


\section{List of figures}

Figure 1.1 Number of publications (article, conference paper, article review, conference review and article in press) with the words "random laser" present in the title, abstract or key-words (sources: IEEE, OSAm and Science Direct).

Figure 2.1 Light-matter interaction forms.

Figure 2.2 Boltzmann distribution. It gives the occupation $P\left(E_{m}\right)$ probability of an arbitrary energy level $E_{m}$.

Figure 2.3 Principle of light amplification by stimulated emission of radiation - laser. An external pumping mechanism is required to provide population inversion such that stimulated emission exceeds absorption.

Figure 2.4 Gain profile (a) and phase shift (b) for a laser amplifier with Lorentzian lineshape.

Figure 2.5 Fabry-Perot resonator: two planar mirrors trap light that bounces at each mirror repeatedly.

Figure 2.6 Intensity profile of a Fabry-Perot etalon resonator. (a) In the absence of losses, only modes at the discrete resonant frequencies are sustained. (b) A lossy resonator sustains waves with small deviations from the discrete resonant frequencies.

Figure 2.7 Laser oscillator: an active medium is inserted in an optical cavity composed of two mirrors, where one is made partially transmitting allowing a portion of coherent light to escape.

Figure 2.8 Variation of gain coefficient with photon-flux density in laser devices. Steady state operation is achieved when $\gamma(\nu)=\alpha_{r} .39$

Figure 2.9 Impacts of gain and phase conditions in the allowed lasing modes.

Figure 2.10 Examples of measured optical spectra for single (left) and multimode (right) lasers [34].

Figure 2.11 Energy diagrams for $\mathrm{Si}$ and GaAs semiconductors. Si exhibits an indirect bandgap, with bandgap energy of $1.12 \mathrm{eV}$, while GaAs has a greater bandgap energy of $1.42 \mathrm{eV}$, and a direct bandgap.

Figure 2.12 Schematic of light amplification by an SOA.

Figure 2.13 (a) Measured amplified spontaneous emission spectrum of SOA1013SXS [38], and (b) its fiber-coupled packaged mount.

Figure 2.14 SOA1013SXS gain dependence with output power for a fixed driving current of $500 \mathrm{~mA}$ [38].

Figure 2.15 Measured SOA gain dependence with driving current. 
Figure 3.1 Schematic representation of the amorphous structure of optical fibers. (a) The arrangement of molecules in silica fibers exhibit an intermediate range order, defining volume elements of constant densities. The transition between two nearly ordered regions is indicated as the border between green and orange regions, the latter encompassing molecules more densely arranged, defining a region with greater refractive index. (b) Schematic of a fiber section in sub-micron scale. Atoms are represented by orange dots, and black lines define regions of constant density.

Figure 3.2 Representation of theoretical model for Rayleigh backscattering intensity fluctuations in single mode fibers. Given the nanometric scale refractive index fluctuations, it is assumed a continuous scattering along sections of length $d$ ( $\sim 1$-cm long), with a local average refractive index $\bar{n}_{i}$ for an $i^{\text {th }}$ section. The centimetric scale index fluctuations, caused by residual stress, results in a different local average index for each section $d$.

Figure 3.3 (a) Simulated intensity of Rayleigh backscattered light for three fiber lengths. (b) FFT results of simulated optical field fluctuations for different fiber lengths.

Figure 3.4 (a) Simulated phase-OTDR trace for a 1-km long fiber with temperature varying up to $0.1{ }^{\circ} \mathrm{C}$ at position $z=200-210$ m. (b) Zoom in the region of interest.

Figure 3.5 Simulation results for phase-OTDR traces considering residual stress imposed at multiple spatial periods, ranging from $0.001 \mathrm{~m}$ to $1,000 \mathrm{~m}$. Amplitudes were normalized by the maximum value.

Figure 3.6 Probability density function of simulated Rayleigh backscattering intensity fluctuations in (a) an 8-km long fiber for different bandwidths and (b) for a fixed bandwidth of 20 $\mathrm{MHz}$ and different fiber lengths (b).

Figure 3.7 Simulated probability density functions of Rayleigh backscattering intensity fluctuations considering the refractive index with a random Gaussian distribution. When the standard deviation $\sigma$ of the index distribution reaches values below $10^{-13}$, it is observed a transition from an exponential PDF to a distribution of a sinusoidal variable.

Figure 3.8 Example of ergodic behavior. (a) Histogram of $x(t)$ for 4,000 samples, and (b) histogram of logistic mapping for 2,000 samples [52].

Figure 3.9 Experimental setup for measurement of intensity fluctuations of Rayleigh backscattering. AOFS, acousto-optic frequency shifter; BPF, band-pass filter; LO, local oscillator; OI, optical isolator; OSC, oscilloscope; PD, photodiode.

Figure 3.10 Spectral measurement of Rayleigh backscattering intensity fluctuations from 1, 2, 4 and $8 \mathrm{~km}$ fibers. 
Figure 3.11 Probability density functions of Rayleigh backscattering intensity fluctuations evaluated in time (experimental), and frequency domains (simulation). Intensities are normalized with respect to the mean value.

Figure 3.12 Experimentally obtained probability density function of Rayleigh backscattering intensity fluctuations for different acquisition intervals (a) and for different fiber lengths (b).

Figure 4.1 Schematic of random lasing [10]. Multiple scatterers traps the light in a confined gain volume, increasing the effective amplification path undergone by light. Open random paths determine an incoherent feedback, whereas closed paths allow coherent feedback. The panel on the left corresponds to a simulation of power spatial distribution [76].

Figure 4.2 Three successive measured spectra of a random laser composed of a porous glass doped with laser dye. The chaotic behavior of emission spectra is observed, where no correlation is found between successive spectral measurements [79].

Figure 4.3 Random distributed feedback fiber laser. An optical fiber provides distributed Raman gain when pumped by two lasers. Distributed Rayleigh backscattering promotes coherent feedback along the optical fiber, defining an open optical cavity. With light trapped in the transverse plane of propagation direction, the distributed feedback and gain mechanisms can generate random laser action, which is thus observed in a onedimension structure [10].

Figure 4.4 Optical spectra of random DFB fiber laser [1]. Pumping near (a) and significantly above the generation threshold (b).

Figure 4.5 Temporal shots of random DFB fiber laser at different pumpings: (a) $49.2 \mathrm{~W}$, (b) $59.2 \mathrm{~W}$, (c) $72.1 \mathrm{~W}$, and (d) $85.6 \mathrm{~W}$ [86].

Figure 5.1 Simulated power reflectivity of an $8 \mathrm{~km}$-long standard single mode fiber.

Figure 5.2 Basic scheme of a random feedback fiber laser with localized gain. FBG: Fiber Bragg Grating; OI: optical isolator; SMF: single mode fiber; SOA: semiconductor optical amplifier PC: Polarization Controller.

Figure 5.3 Simulated phase of the electromagnetic field after a round trip in a random feedback fiber laser as a function of frequency (offset from 194.2 THz). Red dash-dot and green dash lines in the top panel represent additional gains of 3 and $6 \mathrm{~dB}$ provided by a single pass on the SOA. Red crosses in the bottom panel do not represent modes -- they are due to the phase wrapping procedure. Blue points correspond to real solutions of the phase condition. 
Figure 5.4 (a) Simulated cumulative probability of instantaneous cavity loss. (b) Histogram of adjacent modes spacing found for simulated $8 \mathrm{~km}$-long random feedback fiber laser within $1 \mathrm{GHz}$ bandwidth. The average mode spacing of $26.5 \mathrm{kHz}$ is close to the $25.7 \mathrm{kHz}$ value from a uniform feedback fiber laser.

Figure 5.5 Experimental setup of Random Feedback Fiber Laser (left) and measurement setups (right). AOFS: Acousto-Optical Frequency Shifter. DSF: Dispersion Shifted Fiber; ESA: Electrical Spectrum Analyser; FBG: Fiber Bragg Grating; OSA: Optical Spectrum Analyser (High and Low resolutions); OSC: Oscilloscope; PC: Polarization Controller; PD: Photodiode.

Figure 5.6 Variation of the random laser average optical power with SOA driving current for an $8 \mathrm{~km}$-long dispersion shifted fiber (a). SOA end to end gain curve (b).

Figure 5.7 Detected pulses emitted by random lasers at SOA driving currents of $200 \mathrm{~mA}$ (a), $205 \mathrm{~mA}$ (b), $210 \mathrm{~mA}$ (c) and $400 \mathrm{~mA}$ (d), with the corresponding optical spectra in (e), (f), (g) and (h), respectively.

Figure 5.8 Output of random laser showing three overlapping pulses, corresponding to the overlap of three simultaneous modes. A zoom-in on the red rectangle area shows evidence of strong beat notes.

Figure 5.9 (a) Random laser optical spectrum measured in a high resolution (0.16 pm) OSA; (b) Measured and calculated electrical power spectrum. Both measured curves were obtained when driving the SOA with $500 \mathrm{~mA}$.

Figure 5.10 (a) Random laser linewidth for different SOA driving currents; (b) Intensity of the mode mean power and multimode beat noise floor.

Figure 6.1 Experimental setup. AOFS: Acousto-optic frequency shifter; DS: Dispersion shifted; EDFA: Erbium-doped fiber amplifier; ESA: Electrical spectrum analyzer; FBG: Fiber Bragg grating; LO: Local Oscillator; OBPF: Optical band-pass filter; OI: Optical isolator; OSA: Optical spectrum analyzer; OSC: Oscilloscope; PC: Polarization controller; PD: Photodector; VOA: Variable optical attenuator.

Figure 6.2 (a) Brillouin-based RFL output spectrum before and after spectral filtering, and FBG reflectivity profile. (b) Power dependence of Brillouin RFL and output pump laser with input pump power.

Figure 6.3 RFL output measured at two pumping powers (a). Typical laser pulses emitted when pumping near (b) and slightly above (c) the generation threshold.

Figure 6.4 (a) Measurement of RFL spectral content with the delayed self-heterodyne technique for different pumping powers. (b) Peak power increase, and multimode beating power increase with pumping power. 
Figure 6.5 (a) Heterodyne measurement with local oscillator of Brillouin-based RFL. (b) Direct detection of RFL output.

Figure 7.1 (a) Experimental setup of SOA-based random DFB fiber laser with intra-cavity phase modulation. (b) Electro-optic phase modulator. (c) Fiber stretcher phase modulator.

Figure 7.2 Frequency response of high-efficiency fiber stretcher Optiphase PZ2-SM2 [123]. SM2-layer (blue curve) corresponds to the model used in the experiments.

Figure 7.3 Theoretical result for power distribution between a monochromatic carrier and its sidebands after dual phase modulation with in-phase contributions (blue curves). The black curve shows a simulation result for the added loss coefficient for the random DFB fiber laser when operating with in intra-cavity phase modulation with modulation amplitude $A_{m}$.

Figure 7.4 Measurement of random DFB fiber laser output power increase with SOA driving current for different phasemodulation voltages, at a modulation frequency of $100 \mathrm{MHz}$.

Figure 7.5 Characterization of experimental set: double pass at EOPM + FBG. (a) Experimental configuration. (b) Example of spectral measurement at OSA using $f_{m}=100 \mathrm{MHz}$.

Figure 7.6 Comparison between experimental and simulation results for the added loss coefficient. Blue curves show the energy distribution of a theoretical monochromatic wave and its sidebands when under dual phase-modulation. 


\section{List of tables}

Table 3.1 Comparison of light scattering phenomena [40]. 


\section{List of Abbreviations}

AOFS - Acousto-optic frequency shifter

ASE - Amplified spontaneous emission

$\mathrm{BPF}$ - Band pass filter

CRN - Coherent Rayleigh noise

CW - Continuous wave

DCF - Dispersion compensating fiber

DFB - Distributed feedback

DSF - Dispersion shifted fiber

EDFA - Erbium-doped fiber amplifier

EOM - Electro-optic modulator

EOPM - Electro-optic phase modulator

FBG - Fiber Bragg grating

FFT - Fast Fourier transform

FWHM - Full width half maximum

IEEE - Institute of Electrical and Electronics Engineers

LD - Laser diode

LED - Light emitting diode

LO - Local oscillator

MZI - Mach-Zehnder interferometer

OFDR - Optical frequency domain reflectometry

OI - Optical isolator

OPD - Optical path displacement

OSA - Optical spectrum analyzer

OSAm - Optical Society of America

OSC - Oscilloscope 
OTDR - Optical time domain reflectometer

PD - Photodiode

PDF - Probability density function

PDG - Polarization dependent gain

PMD - Polarization mode dispersion

PZT - Piezoelectric transducer

RFL - Random fiber lasers

RIN - Relative intensity noise

SBS - Stimulated Brillouin scattering

SLM - Single longitudinal mode

SM - Single mode

SNR - Signal to noise ratio

SOA - Semiconductor optical amplifier

SRS - Stimulated Raman scattering

TBPF - Tunable band-pass filter

TOBPF - Tunable optical band-pass filter

TWA - Traveling wave amplifier

VBW - Video bandwidth

VOA - Variable optical attenuator

WDM - Wavelength division multiplexing 
I have no special talents. I am only passionately curious.

Albert Einstein. 


\section{1 \\ Introduction}

The report of a novel type of random laser in 2010 by Turitsyn and coworkers [1] have paved the way for the rich research field of random distributed feedback fiber lasers. Since then, random fiber lasers (RFLs) have gained a lot of attention, and many works have been reported in this field, presenting novel configurations, applications, and better comprehension of the complex physical features involved in RFLs. In this thesis we explore a gap in comprehension of RFLs with distributed feedback (DFB) to better understand the mode structure of such lasers. It remains a challenge to understand in details the chaotic emission of random DFB fiber lasers in both temporal and spectral domains, and our main target is to advance in the comprehension of the phenomenon. For that, we propose a novel RFL with DFB in a reduced complexity configuration, which helps to better analyze the laser emission characteristics. We carried out a rigorous investigation to characterize the distributed feedback mechanism. This includes the development of a novel theoretical model to predict intensity fluctuations of Rayleigh backscattered coherent light, which has the potential to characterize the lasing modes dynamic. This chapter introduces the background and motivation of the work, go through the main objectives of the thesis, presents a historical overview of random lasers, details the relevance of the thesis and the need to advance in the field, and list its main contributions. It is organized as follows. Section 1.1 presents the motivation of the work, describing some fundamental aspects regarding laser action in RFL that are yet to be fully understood. Next, in section 1.2 we present the objectives of the work. An overview of the principal historical milestones regarding advances in the study of random DFB fiber lasers is given in section 1.3. Section 1.4 describes the relevance of the work and explains the demand for advancing in both modeling intensity fluctuations of Rayleigh backscattering coherent light, and characterizing the dynamics of random DFB fiber lasers. In section 1.5 we list the main contributions of the thesis. Last, the organization of the thesis is provided in section 1.6. 


\section{1 \\ Motivation and background}

Usually, randomness is an undesired feature in most systems. It means that there is a part of the system or phenomenon under analysis that is not controllable, driving people either to avoid its study or to develop ways to mitigate the impacts of randomness. For instance, in fiber-optic communication systems, the random density fluctuations of the fiber's refractive index result in losses of the propagating signal, often requiring amplification to compensate the losses and extend communication links. From a more philosophical aspect, even Einstein was not satisfied with randomness, and in his later years he strongly rejected quantum indeterminism. On the other hand, organized and deterministic phenomena are much more satisfying, as with the support of mathematical models, one can predict their behavior over a long period of time. There are many examples of materials with organized structures and periodic refractive index that have shown to be relevant for multiple applications in optics. To name a few, semiconductor distributed feedback (DFB) lasers, fiber Bragg gratings (FBGs) and optical filters [2] are devices with periodic refractive index with countless applications in research laboratories and optical systems.

A recent line of research in optics goes against this common viewpoint of randomness, and tries to explore the benefits that a medium with random refractive index can bring. A first argument in favor of working with random refractive indexes is the ease of fabrication, as many photonic materials are naturally found with a fluctuating refractive index. Although the technology for fabrication of photonic devices with periodic refractive index is already mastered for several applications, the fabrication process is still costly. For example, the fabrication of a semiconductor DFB laser requires the inscription of a periodic refractive index along an ultra-precise microcavity, which involves high technology machinery that translates into high production cost.

A second and stronger argument supporting the research of photonic devices with disordered refractive indexes is that they can eventually exceed the performance of man-made periodic devices. This aspect have led researchers to intentionally add, or increase the disorder of the refractive index to obtain improved performance. For instance, random scattering in disordered media has been explored in many spectroscopy applications. It has been shown that random scattering materials are diverse enough in spectral transmission to allow for a precise measurement of the wavelength-dependent state of polarization of an optical field [3]. Another interesting work in random spectroscopy was reported by Redding and co-workers [4]. They made use of multiple light scattering from a silicon-on-insulator chip featuring a random structure to develop 
a compact spectrometer with $0.75 \mathrm{~nm}$ resolution.

The randomness of refractive index has also been used to generate laser emission. Although the term random laser was only introduced in 1995 by Wiersma [5], the concept of random lasing phenomenon was introduced in late 1960s by Ambartsumyan [6], a few years after the development of the first laser. Conventional lasers are normally thought of optical devices with a gain medium combined with a well-defined optical cavity. The cavity acts as spectral filter, allowing the oscillation of a discrete set of modes. By increasing the reflectivity of cavity mirrors, the feedback is increased, what enhances filtering performance with the drawback of reducing the portion of light escaping the cavity. In the firsts random lasers, a disordered medium was introduced in the gain region, promoting incoherent feedback by multiple scatterings. These end up increasing the effective mean optical path within the gain medium, hence boosting light amplification to a point where the gain is higher than the losses, when laser light is observed. In Ambartsumyan work, it was proposed the replacement of one of the mirrors of a ruby laser with a diffuse surface, resulting in multiple scatterings providing incoherent feedback and random laser emission. However, experimental proof of random lasing has only been accepted in 1994, reported in a paper by Lawandy and co-workers [7]. Since the feedback is promoted by scattering centers, it was shown that an increase in the feedback can be obtained by altering the concentration, dimension and composition of scattering particles.

Given the simpler design of random lasers compared to traditional lasers, the former seemed to be a promise for a new generation of laser light sources. Also, as random lasers can be developed in compact structures, such as powders, porous glasses and semiconductor particles [8], the interest in this type of device has grown a lot over the years. However, given the complex emission characteristics of random lasers in the spatial, temporal and spectral domains, they turned out to be less attractive for practical applications.

An approach used to reduce complexity, and, thus, simplify the control of random laser properties, is to confine the spatial emission to lower dimensions. de Matos and co-workers reduced the dimensionality of random laser emission to nearly one when a rhodamine $6 \mathrm{G}$ solution with $\mathrm{TiO}_{2}$ particles was inserted into the hollow core of a photonic crystal fiber, giving rise to the first random fiber laser in 2007 [9]. The $\mathrm{TiO}_{2}$ particles act as scattering centers, providing incoherent feedback and increasing the effective amplification path, while the photonic crystal fiber confines light within its core. Three years later, it was reported the first random distributed feedback fiber laser, with feedback provided by Rayleigh scattering, and distributed gain by Raman effect [1]. 
This paper was a milestone for random laser science, showing that random laser action can occur in a standard single mode fiber even with an extremely low reflectivity given by Rayleigh scattering.

The number of works reporting new configurations of RFLs, analysis of emission characteristics, and novel applications like distributed sensing and random number generation has grown substantially since 2010. To quantify this growth, Figure 1.1 brings the number of publications over the years in IEEE (Institute of Electrical and Electronics Engineers), OSAm (Optical Society of America) and Science Direct journals with the term "random lasers" present in the publication title, abstract or manuscript key-words.

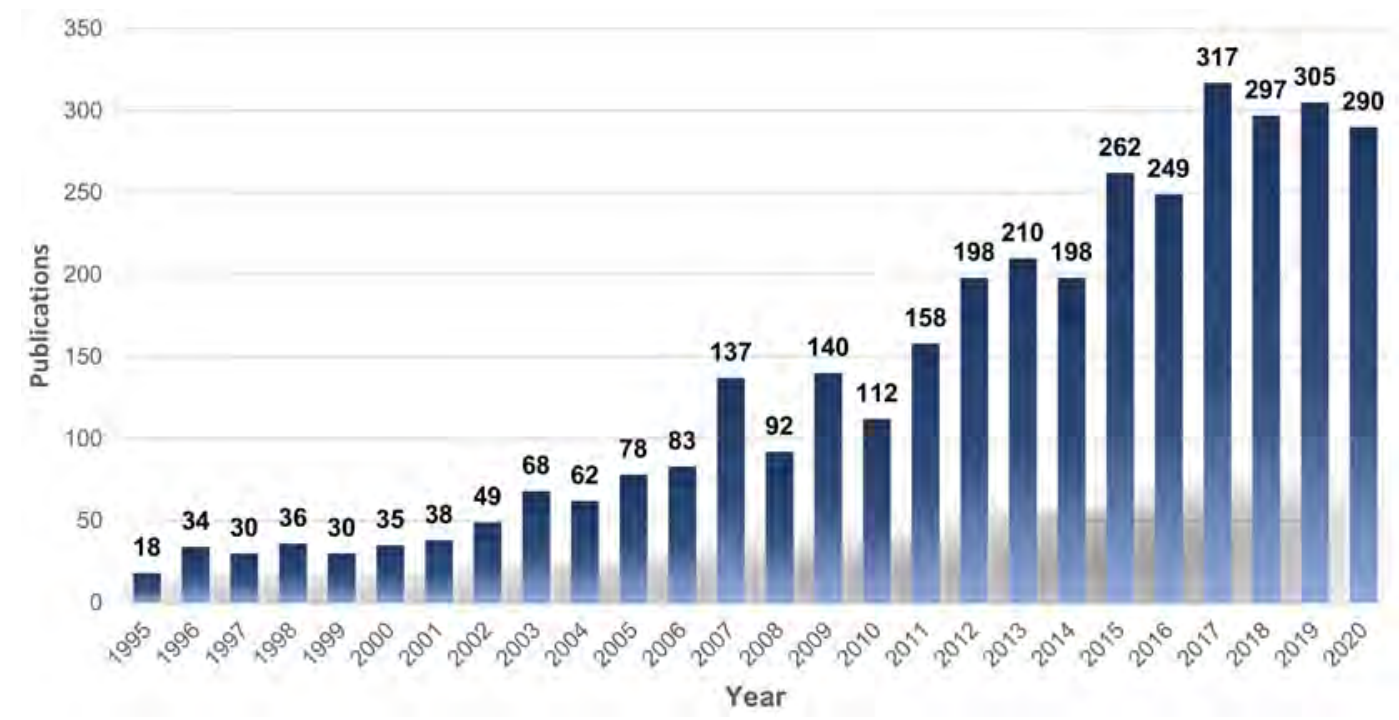

Figure 1.1: Number of publications (article, conference paper, article review, conference review and article in press) with the words "random laser" present in the title, abstract or key-words (sources: IEEE, OSAm and Science Direct).

The reduction in complexity of RFLs is accompanied by an improvement in efficiency. As light is trapped in the fiber's transverse plane, the optical path within the gain medium is highly increased, requiring less pumping to obtain the same output power. The RFL reported in [9] has shown to be more efficient than bulk random lasers by at least a factor of 100. In [1], an efficiency of $30 \%$ was obtained using the random distributed feedback laser previously described, an efficiency comparable to conventional lasers. Later, it was shown that RFLs have efficiency and performance that are not only comparable, but can even exceed those of traditional lasers [10]. Z. Wang and co-workers used a short fiber (less than $1 \mathrm{~km}$ ) to form an open-cavity RFL, and showed that the relative quantum efficiency can exceed $100 \%$ due to the specific longitudinal power distribution along the cavity, which has lower loss compared to the pump wave [11]. 
Despite the high efficiency and simple fabrication/low production cost, RFLs still exhibit complex behavior in both time and spectral domains. For instance, the dynamics of random DFB fiber lasers are characterized by a chaotic pulsed regime and a spiky emission spectrum near the lasing threshold. Although it has been shown that further increase in the pump power of random DFB fiber lasers eventually leads to a stabilization in temporal and spectral domains [1], the transition from spontaneous emission (pumping below threshold level) to a stable stimulated emission (pumping significantly above threshold level) is not fully understood. Actually, this is still a challenge in random laser physics, and in this work we carry a rigorous investigation of the phenomenon. The main motivation of this work is to provide, with the support of theoretical analysis and experimental measurements, a better comprehension of the physics ruling this lasing phenomenon and the mode dynamics involved in the process.

A further reduction in the complexity of random DFB fiber lasers can be obtained by replacing the distributed gain provided by Raman effect with a point-based (localized) gain from a semiconductor optical amplifier (SOA). In this case, as will be discussed in section 4.2.3, gain and feedback mechanisms are decoupled, allowing the analysis of gain and feedback effects separately, easing the comprehension of the chaotic emission of random DFB fiber lasers.

\section{2 \\ Objectives}

The main objective of this work is to advance in the comprehension of the fundamental physics governing the chaotic emission of random DFB fiber lasers. By using a novel experimental setup with point-based gain provided by an SOA $[12,13]$ instead of the commonly used distributed gains, i.e., Raman gain $[1,10,14,15]$, Brillouin gain [16-19] and fiber-doped amplifiers [20-22], gain and feedback are decoupled, allowing us to focus on the gain and feedback effects separately. This simplifies significantly the analysis of the key aspects of random DFB fiber lasers, as for instance complex cooperative effects between Brillouin gain and Rayleigh feedback [23] are not present. Although other works reported the implementation of similar random DFB fiber lasers with SOA as gain medium, the Rayleigh backscattering mechanism was poorly investigated. Thus, before evaluating the complex characteristics of random DFB fiber lasers, we investigate intensity fluctuations of Rayleigh backscattered coherent light in single mode optical fibers.

Therefore, to be able to achieve our main goal, a first objective is put forward: the development of a theoretical model for Rayleigh backscattering 
intensity fluctuations in the frequency domain. However previous works have reported models for Rayleigh backscattering intensity fluctuations in single mode fibers [24-29], none have modeled the phenomenon in the frequency domain. The development of a model in the frequency domain has the power not only to provide means to characterize the mode dynamics of random DFB fiber lasers, but also to predict temperature or stress variations along the fiber, thus, being capable of simulating phase-Optical time domain reflectometer systems ( $\Phi$-OTDR). Similar to polarization mode dispersion (PMD), which has been shown to be a phenomenon exhibiting ergodicity between time and frequency domains [30], we investigate the ergodicity property of Rayleigh backscattering in single mode fibers. This is another important objective of the work, which we analyze by comparing the statistics of model's predictions in the frequency domain with experimental measurements of Rayleigh backscattering intensity fluctuations in time domain. For experimental measurements, we propose a configuration that minimizes the amplitude and phase fluctuations of the optical source, which let us measure pure Rayleigh backscattering intensity fluctuations, not transmitting other sources of noise to the measurement data.

\section{3}

\section{Historical overview}

The study presented throughout this thesis would not have been possible without the effort of many scientists over the years. It has been a long path since the discovery of the photoelectric effect in 1887, to the report of the first random DFB fiber laser in 2010. This section is a form of appreciation of these hard works. Some of the most important milestone events enabling this thesis, involving the development of random lasers and SOAs (gain medium used), are listed below.

- 1887: Heinrich Hertz discovers the photoelectric effect.

- 1905: Einstein, in his miracle year, explains the photoelectric effect.

- 1917: In the paper "On the Quantum Theory of Radiation", Einstein establishes the theoretical basis for lasers and masers, the phenomenon known as Stimulated Emission Radiation.

- 1928: The stimulated emission radiation phenomenon is experimentally confirmed by Rudolf W. Ladenburg.

- 1953: The first maser is produced by Charles Hard Townes. This work was the basis for the construction of oscillators and amplifiers based on 
maser-laser principle, which rendered him, Nikolay Basov and Aleksandr Prokhorov the shared Nobel Prize in Physics, in 1964.

- 1959: The acronym LASER is first published in the paper "The LASER, Light Amplification by Stimulated Emission of Radiation", by Gordon Gould.

- 1960: Theodore H. Maiman produces the first functional laser by using a solid-state flashlamp-pumped synthetic ruby crystal to produce red laser light, at $694 \mathrm{~nm}$ wavelength.

- 1962: The first semiconductor laser diode, made of GaAs, is developed by Robert N. Hall.

- 1966: Ambartsumyan and co-workers observes random lasing action for the first time when one of the mirrors of a ruby laser was replaced with a diffuse surface.

- 1973: Though the first semiconductor optical amplifier (SOA) studies were reported shortly after 1962, Zeilder and Personick were the ones responsible for pioneering work with SOAs in 1973, driven by the advantages of heterojunction devices.

- 1994: An experiment conducted by Lawandy, Balachandran, Gomes and Sauvain with a laser dye dispersed in a strongly scattering medium exhibits the manifestation of laser action. Results indicate that the feedback is proportional to the density of scattering particles.

- 1995: The term random laser is introduced by Wiersma in the paper entitled "Random laser?".

- 2001: A ZnO nanowire under optical pump is shown to exhibit ultraviolet laser action, with light confinement guaranteed by the difference between the $\mathrm{ZnO}$ refractive index and the surrounding environment.

- 2007: de Matos and co-workers inserted a rhodamine solution with $\mathrm{TiO}_{2}$ particles into the hollow core of a photonic crystal fiber to generate the first random fiber laser.

- 2010: The research field of random distributed feedback fiber lasers is open in the seminal paper "Random distributed feedback fibre laser", published by Turitsyn and co-workers. 


\section{4 Relevance}

This thesis is relevant for many fields in optical fibers science, including optical fiber sensing, optical fiber monitoring systems (OTDR and Optical frequency domain reflectometry), optical fiber bi-directional communication and random DFB fiber lasers. The developed model for Rayleigh backscattering in the frequency domain described in chapter 3 can provide information, for instance, of the temperature sensibility of an optical fiber sensor, with extremely high spatial resolution in the order of centimeters. We show that temperature variations as small as $0.01{ }^{\circ} \mathrm{C}$ could, in theory, be predicted with the technique. Compared to previously reported models [24-29], the one presented here shows several advantages, which are discussed in details in section 3.2.2.

In addition, the chaotic emission of random fiber lasers is still a matter of great discussion, and certainly not fully understood. Reported works have attributed the unstable emission to the presence of Brillouin light in the laser cavity in cooperation with Rayleigh scattering $[1,10,14]$. However, by using a tunable optical filter with narrow spectral bandwidth, and by pumping at power levels below the Brillouin threshold, we show that the chaos is present in both temporal and spectral domains even in the absence of Brillouin light. By validating that the laser emission is governed by the statistics of intensity fluctuations of coherent Rayleigh backscattered light, we open the path for the development of random lasers with controlled emission. To validate our predictions, we propose a novel modulation scheme, that controls the laser emission by braking either phase or gain condition for lasing.

Thus, the lack of a model for Rayleigh backscattering intensity fluctuations in the frequency domain, combined with an open challenge in random laser physics, configure an extremely relevant and exciting topic for doctoral research, which we investigate both theoretically and experimentally in this thesis.

\section{5}

\section{Thesis contributions}

The main purpose of this thesis is to investigate the complex characteristics of the dynamics of random DFB fiber lasers. These include mainly the build up of chaotic laser pulses in time domain, the presence of spikes at random wavelengths in the spectral domain and the structure of longitudinal modes. In addition, beyond the analysis of complex characteristics, simple characteristics found in random DFB fiber lasers, as well as in traditional lasers, such 
as power increase with pump power, were carefully examined, revealing new understandings of random DFB fiber laser dynamics. In this context, central contributions of this thesis are listed below.

1. As the distributed feedback mechanism in the random DFB fiber laser is Rayleigh scattering, it is provided a careful investigation of how this phenomenon proceeds in optical fibers. A novel model for intensity fluctuations of Rayleigh backscattered coherent light was developed. The model has advantages compared to other models reported in the literature. First, because it is built in the frequency domain, reducing the complexity involved in modeling time-dependent changes, mostly environmental, that randomly modulate the refractive index along optical fibers, thus affecting Rayleigh backscattered light. Second, the model encompasses both sub-micron and macro fluctuations of the fiber's refractive index, and it is shown that the former are related to density fluctuations along the amorphous structure of optical fibers, while the latter are due to residual stress from the manufacturing process. And last, the model is suited for light sources of any linewidth, and for fibers with any length. This is an important aspect as most models for intensity fluctuations of Rayleigh backscattering are built for OTDR applications, which usually consider light sources with coherence length longer than the OTDR pulse, but far smaller than the fiber length. As will be shown in this thesis, it is this broadness of the model that allows its use for prediction of the dynamics of random DFB fiber lasers, since the contribution of light from every section of the fiber should be considered for feedback purpose.

2. The developed theoretical model for Rayleigh backscattering in optical fibers promoted rich numerical results for a statistical analysis of the phenomenon. The intensity fluctuations of Rayleigh backscattering are evaluated for different simulation bandwidths, and experimentally for different time intervals. It was analyzed the dependence of intensity fluctuations with fiber length, with both experimental and simulation results leading to the same conclusion: Rayleigh backscattering light fluctuates more for longer fibers. The remarkable coincidence between the statistics of experimental fluctuations with those obtained from the theoretical model in the frequency domain validated the ergodicity property of the phenomenon, a relevant result for many research areas.

3. With the support of the developed model for Rayleigh backscattering, the dynamics of a particular random DFB fiber laser were analyzed. It was 
chosen a configuration with half-open cavity, where an FBG provided feedback at one end, and distributed Rayleigh scattering at the other end. An SOA was chosen as the gain medium, mostly because of the simplicity of its gain distribution, what makes it easier to model the laser system and better to perceive fundamental physical aspects, but also because of availability in the laboratory where measurements took place. Using this configuration and the Rayleigh backscattering model, the random laser threshold was defined in a statistical framework.

4. The chaotic pulsed regime, common to many configurations of random lasers, was shown to be due to randomly driven passive Q-switching, induced by a scintillation effect in the Rayleigh backscattering light, which translates as a time-varying cavity loss. Based on the validation of the ergodicity property and on the predictions of the theoretical model, random laser pulses were shown to manifest at random wavelengths, explaining the spiky emission spectrum. Experimental results agree remarkably with theoretical predictions.

5. Regarding the mode structure for the SOA-based random DFB fiber laser, it is experimentally demonstrated that single-mode operation is only possible in pulsed regime at pumping powers close to the threshold. Mode linewidth has been measured to be $\sim 4 \mathrm{kHz}$, agreeing with reported results from similar configurations of SOA-based random DFB fiber lasers. Experimental measurements indicate that mode lifetime is approximately of $1 \mathrm{~ms}$, which is shown to be related to phase fluctuations along the fiber due to environmental changes. Under high pumping, temporal measurements of laser emission shows that multimode operation prevails, however linewidth measurements can misleadingly suggest otherwise. The increase in the noise floor in self-heterodyne linewidth measurements, also observed in works from other authors but not explained, is here clarified. It is associated with more modes satisfying the gain and phase conditions at the same time, and their beating gives rise to the noise floor. This explanation is supported by theoretical simulation results, which showed that under high gains, a nearly continuous beating of modes should be observed, and also by temporal measurements, presenting mode beating evidences.

6. Investigation of mode dynamics is also given for random DFB fiber lasers with distributed gain, and we show that most of the mode properties observed for the SOA-based random laser are also found in Brillouinbased random lasers. Last, an important contribution is given when ex- 
amining the coherence aspect of such lasers. By applying intra-cavity phase-modulation we provide experimental proof that coherent feedback is pre-requisite for laser action. Although in theory uni-dimensional random fiber lasers naturally provide coherent feedback, by experimentally breaking the phase-condition for lasing we confirm theoretical expectations. This evidence is relevant since it provides means to control laser emission of random DFB fiber lasers without altering its gain, i.e., by simply breaking the phase-condition.

\section{6}

\section{Thesis outline}

This thesis targets the analysis of the dynamics of a special configuration of random DFB fiber laser, with an SOA providing gain in a localized spot. A theoretical model is developed for the feedback mechanism, and several experiments were undertaken. The comprehension of random laser theory is required for a thorough understanding of the work. Also, a background in laser physics and light scattering phenomena is important for the development of the feedback model. Thus, this thesis is organized as follows.

Chapter 1 contextualizes the work presented in this thesis, describing the main motivations of the research, objectives, relevance and main contributions. An historical overview is also provided and the chapter ends with the outline of the work.

Chapter 2 reviews the main concepts involved in laser theory, setting the stage to discuss random fiber lasers. This chapter is important for the work as a parallel with traditional lasers is traced along the thesis, contributing to an easier understating of theoretical and experimental results in the following chapters. In addition, the chapter presents a review of semiconductor optical amplifiers, which are fundamental devices for this work as will be seen in later chapters.

Chapter 3 proposes and validates a theoretical model for Rayleigh backscattering intensity fluctuations. It starts with an overview of light scattering phenomena. Then, focus is given to Rayleigh scattering, and a review of state of the art models reported in the literature for Rayleigh backscattering in optical fibers is provided. The chapter also presents a novel theoretical model proposed in this thesis, where differences and advantages over other models are highlighted. Simulations are performed to evaluate model predictions. Last, an overview of the ergodic theory is presented, and by comparing simulation results with experimental measurements of Rayleigh backscatter- 
ing intensity fluctuations, we provide strong evidence that the phenomenon exhibits ergodicity in time and frequency domains.

Chapter 4 reviews the theory of random lasers and presents the current stage of the technology. The chapter starts reviewing the more fundamental aspects of random lasers, then discusses the coherence aspect of random lasers, and describes important configurations reported in the literature as well as interesting applications. Chapter ends with a review of random DFB fiber lasers, where SOA-based random DFB fiber lasers are introduced.

Chapter 5 describes the central experiments of this thesis, where fundamental questions regarding the dynamics of SOA-based random DFB fiber lasers are discussed. First, the experimental setup of the proposed random laser is presented. Then, the theoretical model derived in section 3.2.2 is exploited, and we make use of its predictions to explain laser action. The chapter ends with a discussion of spectral measurement results, in both optical and electrical domains.

Chapter 6 investigates the mode dynamics of random fiber lasers with distributed gain provided by stimulated Brillouin scattering. Given the many differences between Brillouin and SOA gains, one might expect the mode dynamics of random fiber lasers based on these two different gain mechanisms to diverge. Multiple experimental analysis were performed, indicating modes in Brillouin-based RFL with a similar dynamics of that observed in chapter 5 , with differences being related to the narrow spectral gain bandwidth of stimulated Brillouin scattering.

Chapter 7 shows that laser emission can be controlled either by breaking gain condition or phase-condition with intra-cavity phase-modulation. Important results obtained in this chapter experimentally proofs that, as expected from uni-dimensional random DFB fiber lasers, the feedback is coherent.

Chapter 8 summarizes the work and suggests possible directions for future research. 


\section{2}

\section{Laser background}

Laser devices were theoretically proposed in 1958 by Schawlow and Townes in [31], and many different lasers have been developed ever since. The list is vast, including 1) lasers at different states - in solids (crystals, glasses, fibers and powders), liquids (organic-dye solutions), gases (atomic, ionic, molecular and excimeric) and plasma (mostly in the extreme-ultraviolet and X-ray region); 2) lasers with a wide range of output power, reaching up to $10 \mathrm{PW}$ - thanks to concepts in the seminal paper of Donna Strickland and Gerard Mourou in 1985 [32], granting them the Nobel Prize in Physics in 2018; 3) lasers operating at multiple wavelengths; and 4) lasers in continuous or pulsed emission. Currently, lasers are found in countless applications, encompassing the following fields: (i) science - photonics, opto-electronics, photochemistry, microwave generation; (ii) medical - eye surgery, dentistry, physiotherapy, dermatology, cancer treatment; and (iii) industrial - optical communications, lidar, laser cut, laser fusion, barcode reader, laser pointer.

As this thesis focus on the study of random DFB fiber lasers, it is important to establish first a background on laser physics. It will be shown in chapter 4 that traditional lasers and random lasers, however different in many aspects, share the same building blocks. This chapter reviews the key concepts of laser devices, and it is organized as follows. In section 2.1 the necessary conditions for laser action are defined, including concepts of light amplification, optical resonators, and phase/gain conditions. Given the importance of semiconductor optical amplifiers for this thesis, section 2.2 provides a short introduction of semiconductor lasers, and reviews the main characteristics of SOAs.

\section{1}

\section{Laser physics}

The term laser is an acronym for light amplification by stimulated emission of radiation. Stimulated emission is a form of light-matter interaction that amplifies a lightwave. As will be shown in the following sections, a laser is an optical oscillator, comprising light amplification in an optical cavity. This section starts with a review of the light amplification mechanism and optical 
resonators, and ends with a combination of these two factors which permit laser action.

\subsection{1}

\section{Light amplification}

Light amplification comes from the fact that light and matter interact with each other. The interaction occurs because matter contains electric charges, which vibrate in the presence of an electric-field and may cause an atomic transition between two energy levels, when light can be either emitted or absorbed. Let $E_{1}$ and $E_{2}$ be subsequent energy levels of atoms enclosed in a volume $V$, such that $E_{2}>E_{1}$. Three possible light-matter interactions can take place, spontaneous emission, absorption and stimulated emission, which are schematically shown in Figure 2.1.

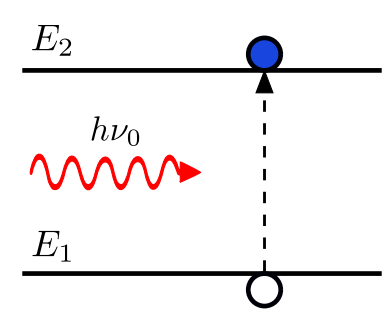

(a) Absorption

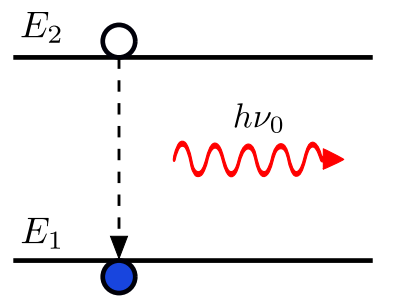

(b) Spontaneous emission

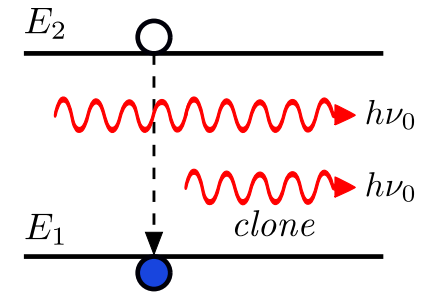

(c) Stimulated emission

Figure 2.1: Light-matter interaction forms.

As the name suggests, spontaneous emission is the only interaction that occurs spontaneously, not requiring the presence of an incident photon. Atoms at energy level $E_{2}$ might spontaneously decay to $E_{1}$, and a photon of energy $h \nu_{0}=E_{2}-E_{1}$ is emitted in the process, where $h$ is Planck's constant and $\nu_{0}$ the optical frequency of the emitted photon. This phenomenon is exemplified in Figure 2.1(b). The probability density $P_{s p}$ for spontaneous emission to occur is given by [33]:

$$
P_{s p}=\frac{1}{t_{s p}}
$$

where $t_{s p}$ is the spontaneous lifetime of the transition between energy levels $E_{2}$ and $E_{1}$. Depending on the atomic properties and surrounding environmental conditions, the spontaneous lifetime can vary from subpicoseconds to minutes. Note that $P_{s p}$ has dimension of $\left[\mathrm{s}^{-1}\right]$, and it can be interpreted as the rate at which photons are spontaneously emitted.

Absorption and stimulated emission are transitions induced by incident light. When in the presence of a photon with energy $h \nu_{0}$, an atom at the energy level $E_{1}$ will absorb the photon energy and transition to the higher energy level $E_{2}$ with a given probability. In this process, the incident photon is said to be 
annihilated, and the input light is attenuated. On the other hand, there is a probability that the incident photon will stimulate an atom at the upper energy level to decay to the lower energy level emitting what is called a clone photon. This nomenclature is given because the emitted photon shares the same characteristics of the incident photon (frequency, direction, polarization). This process is responsible for light amplification. These two induced transitions are represented in Figs. 2.1(a) and (c). Next, the probabilities of absorption and stimulated emission are derived.

Let $\phi$ be the photon-flux density [photons $/ \mathrm{cm}^{2}$-s] of a stream of photons with energy $h \nu$ impinging on the volume $V$. If $I$ is the intensity of the photon stream, then the photon-flux density is written as:

$$
\phi=\frac{I}{h \nu} \text {. }
$$

By definition, the probability density for absorption is equal to that of stimulated emission, i.e. $P_{a b} \equiv P_{s t}$. These quantities are often expressed by $W_{i}$ [33], and depend directly on the photon-flux density. This probability density can be expressed by

$$
W_{i}=\phi \sigma(\nu),
$$

where $\sigma(\nu)$ is the atomic transition cross section. It has a frequency dependence because only photons with frequencies nearly matching the energy gap frequency $\nu_{0}$ will interact. The dimension of $\sigma(\nu)$ is $\left[\mathrm{cm}^{2}\right]$, and it can be thought of the effective atomic area capable of "capturing" a photon for interaction.

Although the probability density, or rate, in which photons are absorbed and stimulatedly emitted is the same, the average rate of absorbed photons is different than that of emitted by stimulated emission. The former depends on the number of atoms per unit volume available at the lower level, $N_{1}$, while the latter depends on the same number but available at the higher level, $N_{2}$. Thus, provided that $N_{2}>N_{1}$, or that the population difference $N=N_{2}-N_{1}>0$, then the medium acts as an amplifier. If that is the case, then the medium is operating under population inversion, which is a necessary condition for optical amplification. A medium with population difference $<0$ results in more absorption than stimulated emission, thus it acts as an attenuation device. When $N=0$, the medium is said to be transparent, as the generation of photons equals the annihilation.

At this point, it becomes evident that to obtain optical gain, the condition of population inversion must be satisfied, i.e., there should be more atoms at a higher energy level than at lower one. Under thermal equilibrium condition at a temperature $T$, the probability of an atom occupying an energy level $E_{m}$ is given by the Boltzmann distribution: 


$$
P\left(E_{m}\right) \propto \exp \left(\frac{-E_{m}}{k T}\right),
$$

where $k$ is the Boltzmann constant, and $m$ is a positive integer, representing the quantization of energy levels. This distribution is shown in Figure 2.2.

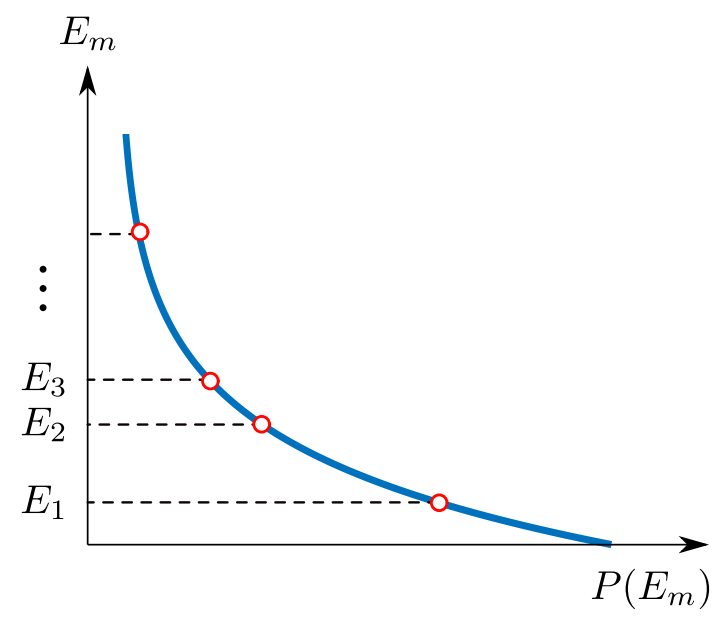

Figure 2.2: Boltzmann distribution. It gives the occupation $P\left(E_{m}\right)$ probability of an arbitrary energy level $E_{m}$.

As it can be seen from Eq. 2-4 and Figure 2.2, under thermal equilibrium, the occupation probability of an arbitrary level $E_{i}$ is always greater than that of a higher energy level $E_{i+1}$. Thus, the condition of population inversion, and hence light amplification, can only be satisfied under non-equilibrium condition. To satisfy population inversion, an external pumping is required, which serves the purpose of populating energy level $E_{1}$ with a rate $R_{1}$, and emptying $E_{2}$ at a rate $R_{2}$.

The pumping mechanism can be realized optically, electrically or chemically. Whatever is the pumping method, it is desired to have a large $R_{1}$ and an also large $R_{2}$, such that there are always atoms at the higher energy level available for stimulated emission, and unoccupied sates at the lower energy level for recombination. With the same principle, it is also important that the energy state $E_{2}$ is long-lived, i.e., its lifetime $\tau_{2}$ is long, meaning that atoms will not rapidly decay for a lower energy level by means of radiative or nonradiative recombination; and that the lifetime $\tau_{1}$, correspondent to the lower energy level $E_{1}$, is short. Figure 2.3 exemplifies this pumping method and light amplification by stimulated emission.

It is relevant to mention two important characteristics of the optical gain mechanism given by stimulated emission: the gain profile and phase shift. As shown in Eq. 2-3, the probability density for absorption/stimulated emission is a function of the transition cross section, which is frequency-dependent. In the same way, the gain coefficient $\gamma$ will also depend on the frequency of incident photons, and it is shown to be [33]: 


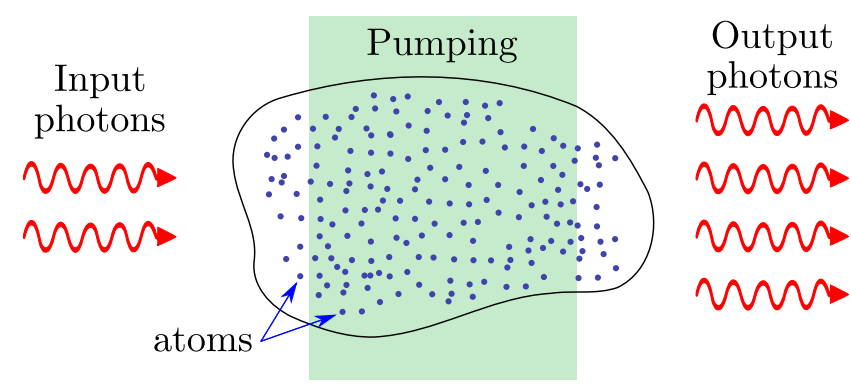

Figure 2.3: Principle of light amplification by stimulated emission of radiation - laser. An external pumping mechanism is required to provide population inversion such that stimulated emission exceeds absorption.

$$
\gamma(\nu)=N \sigma(\nu)
$$

The gain coefficient is centered at frequency $\nu_{0}$, providing maximum gain for photons with frequency $\nu=\nu_{0}$. A much inferior gain is given for photons with frequencies deviating from $\nu_{0}$, the farther is the deviation, the lower is the gain. Figure 2.4(a) shows an example of gain profile with Lorentzian shape. The full width half maximum (FWHM) value of the gain profile is called the transition linewidth, and it will be henceforth referred to as $\Delta \nu$. There are a few factors that impact on the broadening of $\Delta \nu$, which can be either homogeneous or inhomogeneous. When all atoms of the gain medium are considered to be the same, then the broadening is homogeneous. Examples of homogeneous broadening effects are lifetime and collision broadening. If, on the other hand, the active medium is composed of different atoms, which is the case for doped solid systems, then the broadening is inhomogeneous.

An interesting effect that comes from the definition of Eq. 2-5 is gain saturation. The population difference $N$ is a quantity that depends on the photon-flux $\phi$, such that for a high photon-flux the population difference decreases, exhibiting a saturation profile. In the same manner, the gain coefficient saturates for a high photon-flux, and it can be written in terms of the saturation photon-flux density $\phi_{s}(\nu)$ by

$$
\gamma(\nu)=\frac{\gamma_{0}(\nu)}{1+\phi / \phi_{s}(\nu)}
$$

where $\gamma_{0}(\nu)$ is the steady state gain coefficient (in the absence of amplifier radiation).

A gain profile that is frequency selective naturally imparts a phase-shift that is also frequency-dependent. It has been shown that, for a gain profile with Lorentzian shape, the phase-shift coefficient $\varphi(\nu)$ is given by [33]

$$
\varphi(\nu)=\frac{\nu-\nu_{0}}{\Delta \nu} \gamma(\nu)
$$


The frequency-dependence of the phase-shift coefficient for a Lorentzian gain profile is shown in Figure 2.4(b).

(a)

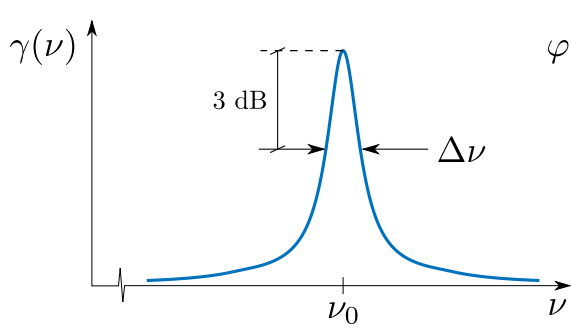

(b)

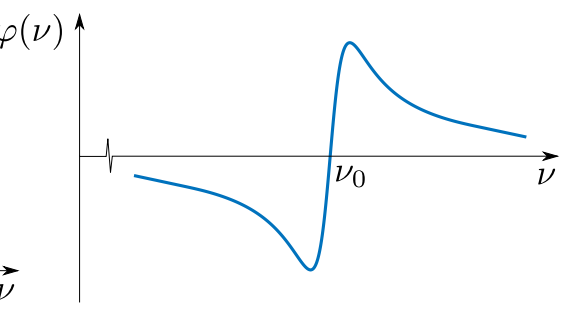

Figure 2.4: Gain profile (a) and phase shift (b) for a laser amplifier with Lorentzian lineshape.

Any optical field with frequency $\nu_{0}$ impinging on an active medium with gain and phase-shift coefficients as per shown in Figure 2.4, would experiment light amplification without phase-shift. That being the case, the active medium is called a coherent optical amplifier. If the phase is not preserved on the amplification process, then the medium is an incoherent optical amplifier. Laser amplification is characterized by being a coherent amplification process, which, as will be seen in the foregoing sections, is a required characteristic for laser action.

\subsection{2}

\section{Optical resonator}

An optical resonator is a component that traps light allowing only a discrete number of frequencies to oscillate in its interior. There are multiple configurations of optical resonators, including planar-mirrors, spherical-mirrors, ring-mirrors, rectangular cavity, fiber-ring, integrated-optic-ring, microshpere, among others [33]. Planar-mirrors, or Fabry-Perot etalon, are the simplest configuration of optical resonators, composed of two planar mirrors separated by a distance $d$, as shown in Figure 2.5.

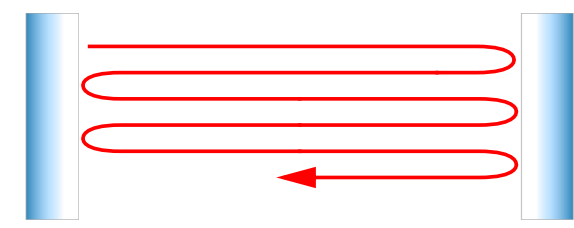

Figure 2.5: Fabry-Perot resonator: two planar mirrors trap light that bounces at each mirror repeatedly.

To calculate the allowed frequencies within the resonator, a wave optics approach is employed. Let $u(\mathbf{r}, t)$ be the wave function of a monochromatic lightwave with frequency $\nu$, such that 


$$
u(\mathbf{r}, t)=\operatorname{Re}\{U(\mathbf{r}) \exp (j 2 \pi \nu t)\},
$$

where $\mathbf{r}$ and $t$ represent the spatial and time dependencies, respectively, and $U(\mathbf{r})$ is the complex amplitude of $u(\mathbf{r}, t)$. To oscillate between the two planar-mirrors, the complex amplitude must satisfy the Helmholtz equation, $\nabla^{2} U(\mathbf{r})+k^{2} U(\mathbf{r})=0$. Assuming that mirrors are normal to the $z$ direction, a solution for this equation is the standing wave $U(\mathbf{r})=A \sin (k z)$, provided that the boundary conditions, $U(\mathbf{r})=0$ at $z=0$ and $z=d$, are satisfied. The first condition is naturally satisfied for every $k$, whereas the second is valid only for a discrete number of $k$ 's: $k_{q}=q \pi / d$, where $q$ is a positive integer. These solutions are called longitudinal modes of the resonator, each of which assuming a different mode number $q$. Alternatively, this condition can be written in terms of optical frequency, i.e.:

$$
\nu_{q}=q \frac{c}{2 d}
$$

where $\nu_{q}$ are resonating frequencies in the optical cavity. An important quantity taken from Eq. 2-9 is the separation between two adjacent resonant frequencies, which is simply given by $\nu_{F}=c /(2 d)$.

Another way to interpret resonator modes is to think about waves that preserve their phase after a round trip in the optical cavity. Although the phase is preserved for the resonating frequencies, the amplitude of the lightwave is reduced due to losses in the cavity. The overall intensity loss is a combination of two factors: the mirrors reflectivity and losses in the cavity medium (mostly absorption and scattering). These losses can be written in the form of an intensity attenuation factor, $|r|$, such that $|r|<1$. Thus, the light intensity in the cavity is a frequency-dependent quantity that also depends on the attenuation factor $|r|$, and it is given by [33]:

$$
\begin{aligned}
& I=\frac{I_{\max }}{1+(2 \mathcal{F} / \pi)^{2} \sin ^{2}\left(\pi \nu / \nu_{F}\right)}, \\
& \text { where: } \quad I_{\max }=\frac{I_{0}}{(1-|r|)^{2}}, \quad \text { and } \quad \mathcal{F}=\frac{\pi \sqrt{|r|}}{1-|r|} .
\end{aligned}
$$

In the equations above, $I_{0}$ is the intensity of the initial wave (before round trip and losses), $I_{\max }$ the maximum optical intensity, which is obtained at the resonant frequencies, and $\mathcal{F}$ is the finesse of the resonator. The finesse is a parameter that characterizes the quality of the resonator. In the absence of losses, $|r|=1$, and the finesse goes to infinity, resulting in an ultra-precise resonator, sustaining lightwaves only at the discrete frequencies governed by Eq. 2-9. This impractical example is shown in Figure 2.6(a), where modes' 
spectral width $\delta \nu$ (FWHM) is zero. In a real oscillator, the presence of losses reduces the finesse, and frequencies with short deviations from $\nu_{q}$ are also sustained. Thus, the spectral width of resonant modes increases with losses, and it can be approximated by

$$
\delta \nu \approx \frac{\nu_{F}}{\mathcal{F}}
$$

(a)

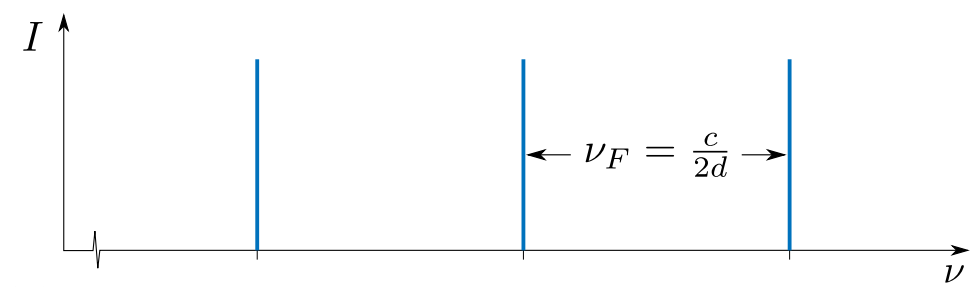

(b)

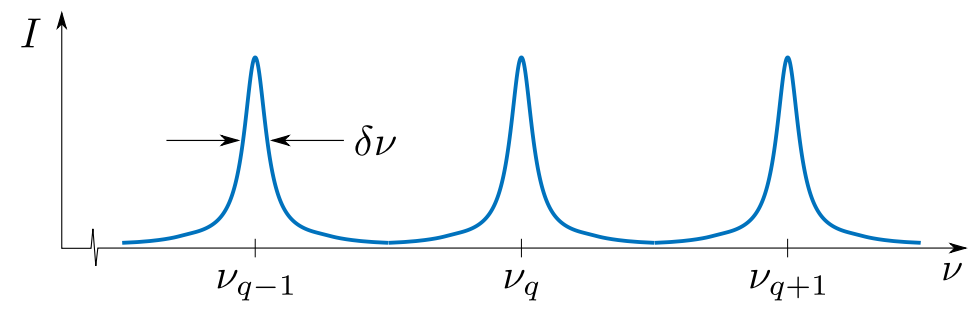

Figure 2.6: Intensity profile of a Fabry-Perot etalon resonator. (a) In the absence of losses, only modes at the discrete resonant frequencies are sustained. (b) A lossy resonator sustains waves with small deviations from the discrete resonant frequencies.

The finite spectral width, caused by losses in the cavity, can be seen as an uncertainty of resonant modes frequencies. This frequency uncertainty translates into time domain by means of the resonator lifetime, or photon lifetime, $\tau_{p}$. It is simple to trace this parallel as the losses in the resonator represent an uncertainty of how long photons will live in the resonator. The relation between spectral width and photons lifetime is given by

$$
\delta \nu=\frac{1}{2 \pi \tau_{p}} .
$$

Last, it will be useful for the following sections to define a loss coefficient, which is related to the attenuation coefficient $|r|$. As mentioned before, the mirrors reflectivity and attenuation along the cavity medium shall be considered in the expression of $|r|$. The overall loss coefficient $\alpha_{r}$ and the attenuation coefficient are related by [33]

$$
\begin{aligned}
& |r|^{2}=\exp \left(-2 \alpha_{r} d\right), \\
& \text { where } \quad \alpha_{r}=\alpha_{s}+\frac{1}{2 d} \ln \left(\frac{1}{\mathcal{R}_{1} \mathcal{R}_{2}}\right),
\end{aligned}
$$


with $\alpha_{s}, \mathcal{R}_{1}$ and $\mathcal{R}_{2}$ corresponding to the medium's loss coefficient, and reflectances of the two mirrors, respectively.

\subsection{3}

\section{Laser action}

A laser is a coherent light source composed of an optical oscillator. The oscillation process is certainly not restricted to optical systems, but any oscillator requires a form of amplification and positive feedback. In laser devices, oscillation is obtained by placing an active medium in an optical resonator. The resonator sustains optical feedback for a discrete set of modes, and the active medium provides optical gain through stimulated emission. Similar to section 2.1.2, for simplicity, it is considered a Fabry-Perot etalon as the optical resonator. However, in this case, one of the mirrors is made partially transmitting such that a portion of light is transmitted. Figure 2.7 presents the building blocks of a laser device, and the laser action process is described as follows.

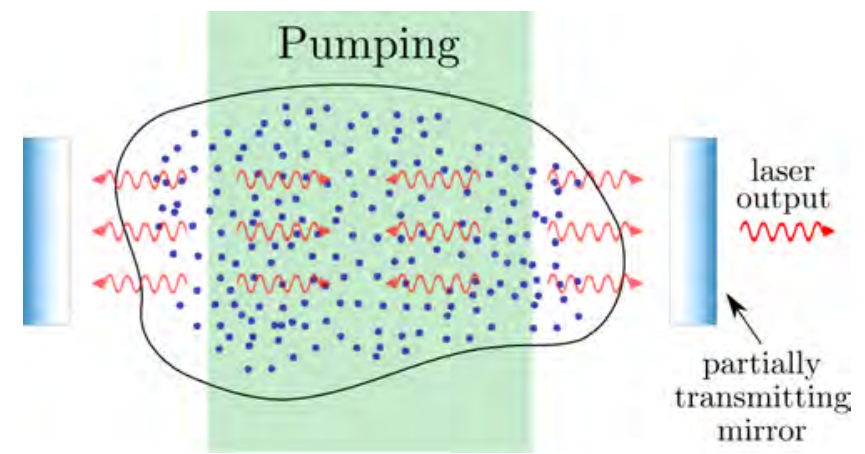

Figure 2.7: Laser oscillator: an active medium is inserted in an optical cavity composed of two mirrors, where one is made partially transmitting allowing a portion of coherent light to escape.

As shown in section 2.1.1, the active medium can emit photons spontaneously or stimulatedly. When a laser device is sufficiently pumped, spontaneously emitted photons starts the lasing process. These photons will induce the generation of clone photons by means of stimulated emission in the active medium. Given the feedback at the two mirrors, the generated photons will traverse the active medium multiple times, each time inducing the generation of more photons, rapidly increase the photon-flux in the cavity. Eventually, for a large photon-flux, the gain saturates, and light is coherently emitted in a steady state regime.

The operation described above holds only if two conditions are satisfied: the gain and phase conditions. The gain condition requires that there is more 
gain than losses in the optical cavity, or, that the gain coefficient at steady state is higher than the loss coefficient, i.e.

$$
\gamma_{0}(\nu)>\alpha_{r}
$$

Note that this condition is stronger than that for light amplification, which demands only that $N>0$ (population inversion). For instance, $N$ can be greater than zero but the losses at the mirrors contribute to a scenario with more losses than gain in the cavity, precluding laser oscillation.

The gain condition and laser emission in a steady state regime are represented in Figure 2.8. Provided that $\gamma_{0}(\nu)>\alpha_{r}$, laser action initiates, and photons start being emitted. Then, the photon-flux density increases and the gain coefficient starts to saturate. With further increase in the photon-flux, the gain decreases to the point where $\gamma(\nu)=\alpha_{r}$, when steady state regime is achieved. At this point, it is said that the gain clamped to the cavity loss.

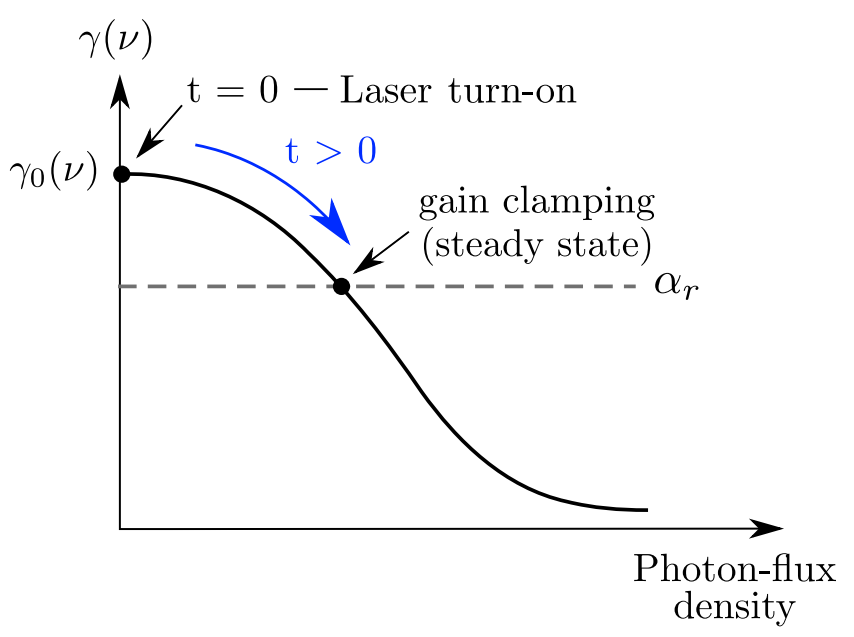

Figure 2.8: Variation of gain coefficient with photon-flux density in laser devices. Steady state operation is achieved when $\gamma(\nu)=\alpha_{r}$.

The other necessary condition for laser action is the phase condition. It requires that the phase of a lightwave is preserved after a round-trip in the cavity. If the contribution of active medium to the round-trip phase is small compared to the distance between the two mirrors, which defines the optical resonator (see section 2.1.2, then the phase condition can be written as:

$$
2 k d=2 \pi q,
$$

where $q$ is a positive integer. Figure 2.9 shows the effects of gain and phase conditions combined, where it can be seen that a stronger restriction is imposed over that shown in Figure 2.6. Even with a dual-restriction, multiple longitudinal modes can still be sustained. In this case, the laser is called a 
multimode laser, whereas if only one mode is sustained, it is referred to as a single longitudinal mode (SLM) laser.

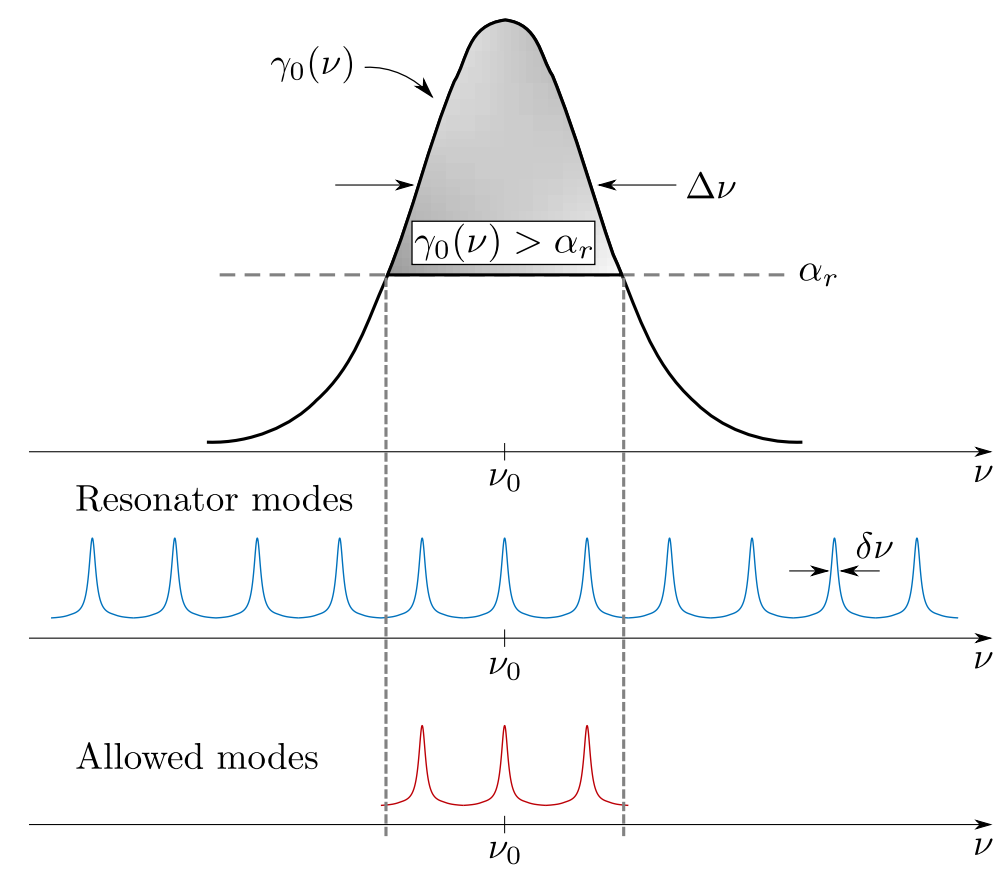

Figure 2.9: Impacts of gain and phase conditions in the allowed lasing modes.

Given the importance of having a single longitudinal mode operation, many approaches have been employed to achieve such condition, examples are modulation of the loss profile, periodic modulation of the refractive index (laser DFB and DBR - distributed Bragg reflectors), coupling light to an external cavity, among many others [2]. Figure 2.10, taken from Peterman's work [34], shows typical optical spectra of single and a multimode lasers. Although a distinction between these two traditional lasers seems quite obvious, extending these concepts for random lasers can be misleading.

\section{2}

\section{Semiconductor optoelectronics}

Semiconductors are solids with electrical conductivity in between that of insulators and metals. Because their conductivity is highly controllable, being altered with temperature, illuminating light or changing the doping composition, semiconductors became the fundamental building block of most applications in electronics. For example, transistors, developed in late 1940's by Shockley, Bardeen and Brattain, are semiconductors currently found in the majority of electronic devices. Beyond applications in electronics, which is defined as the technology of controlling the flow of electric charges (electrons and holes) [33], semiconductors are particularly attractive for controlling the flow of photons, i.e., in photonics. Thus, semiconductors can be thought as a 


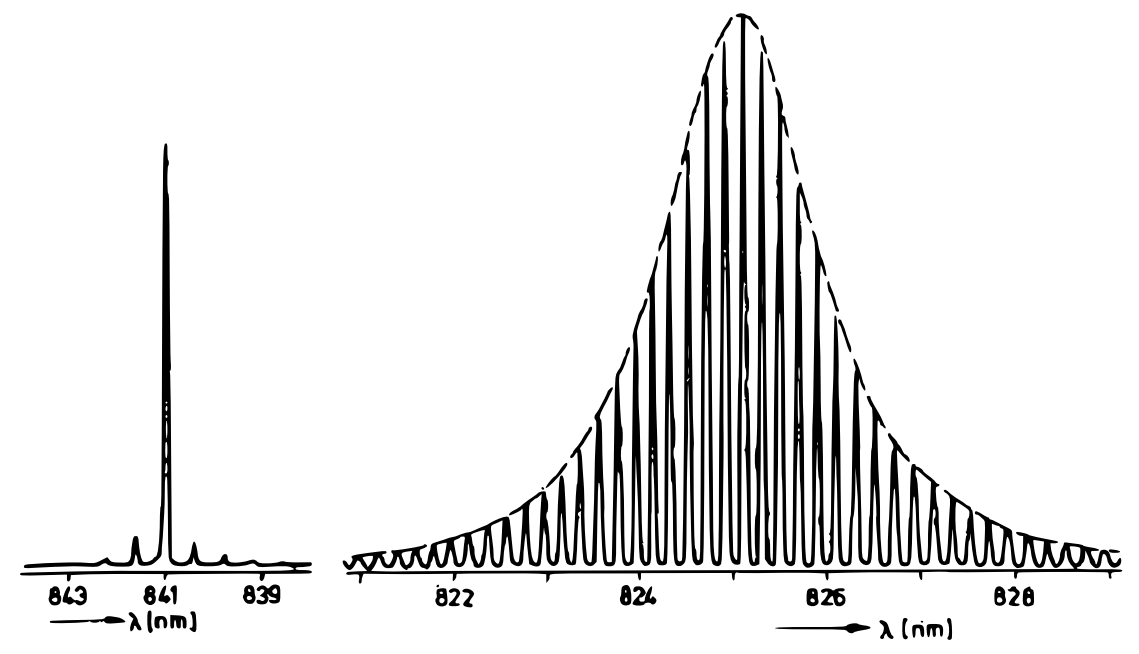

Figure 2.10: Examples of measured optical spectra for single (left) and multimode (right) lasers [34].

link between optics and electronics, giving rise to the field of semiconductor optoelectronics.

Light emitting diodes (LEDs) and laser diodes are examples of semiconductor optoelectronic devices, which emit light under certain circumstances. The emission of photons by semiconductor devices cannot be defined formally in the same way as in section 2.1, where isolated energy levels were considered. Because atoms are closer together in semiconductors, the energy levels associated with the conduction and valence bands broadens into a collection of dense energy levels, forming energy bands. Depending on the semiconductor material, the relation between the energy $E$ of a given band and the wavevector $\mathbf{k}$ vary in different ways. However, close to the top of the valence band, and the bottom of the conduction band, the dependence of $E$ with $\mathbf{k}$ can be approximated by a parabola. An example of $E-\mathbf{k}$ relation for $\mathrm{Si}$ and $\mathrm{GaAs}$ semiconductors is shown in Figure 2.11. When conduction and valence bands of semiconductors are aligned, i.e., the minimum energy of the conduction band and maximum energy of the valence band occur for the same $\mathbf{k}$, then an excited electron occupying the conduction band might undergo a radiative transition and decay to the valence band, emitting a photon in the process and leaving a hole (vacancy) in the conduction band. In the same way as shown in Figure 2.1, the emission can be spontaneous or stimulated by an incident photon. Also, an electron at the valence band might absorb the energy of an incident photon and be excited to the conduction band, leaving a hole in the valence band. On the other hand, if conduction and valence bands are not aligned, a phonon is involved in the process, such that energy is conserved. In this case, it is said that the semiconductor has an indirect bandgap, whereas for aligned bands, the bandgap is direct. 

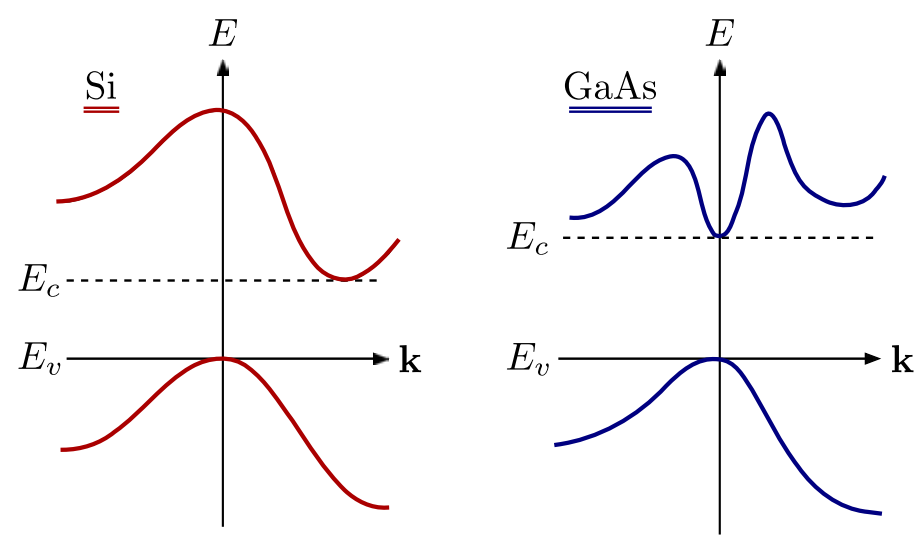

Figure 2.11: Energy diagrams for Si and GaAs semiconductors. Si exhibits an indirect bandgap, with bandgap energy of $1.12 \mathrm{eV}$, while GaAs has a greater bandgap energy of $1.42 \mathrm{eV}$, and a direct bandgap [33].

It is not the purpose of this thesis to provide a thorough review of semiconductor devices, which can be currently found in several books $[35,36]$. Yet, it is specially important for this work to highlight some key properties of semiconductor optical amplifiers. In the following section, a review of such properties is provided, as well as experimental measurements to characterize the gain of the SOA.

\subsection{1}

\section{Semiconductor optical amplifier}

An SOA is an optoelectronic device that amplifies light under suitable conditions. A simple schematic diagram of an SOA is shown in Figure 2.12. By injecting electric current onto the device, its conduction band is populated such that an input light signal can be amplified by stimulated emission. The current is injected in a specific region, called active region, where an embedded waveguide structure is used for light confinement. Different than lasers, which have a cavity defined by a reflection mechanism, reflections are undesired in SOAs as they translate into losses in the output signal. In SOAs it is desired to have a high gain in a single pass of a traveling light wave, so a high reflection at the output end would compromise the net gain. To minimize reflection losses, SOAs are designed with anti-reflection coatings. SOAs with reflectivities lower than $0.1 \%$ are classified as traveling-wave amplifiers (TWA) [2], whereas those with higher reflectivities are classified as Fabry-Perot SOAs [37].

Beyond low facet reflectivities, there are several other properties desired in SOAs. An ideal optical amplifier would exhibit a high gain over a wide bandwidth, be insensitive to polarization, with low noise figure and high saturation power. Typically, SOAs provide lower gain than fiber-amplifiers, with gain extending up to $\sim 30 \mathrm{~dB}$ [37]. The gain bandwidth of SOAs is usually 


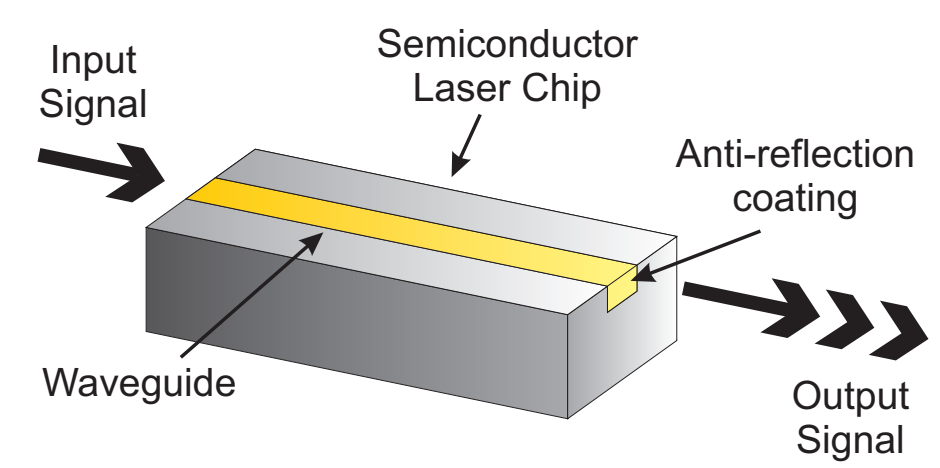

Figure 2.12: Schematic of light amplification by an SOA.

of the order of $30 \mathrm{~nm}$, and it can be easily measured in the absence of an input light signal. Without an input light, the electrons populating the conduction band will decay spontaneously, emitting light in a broadband spectrum, multidirectional and unpolarized. As the spontaneously generated photons traverse the SOA, they experiment amplification along its length (hundreds of $\mu \mathrm{m}$ ), such that the emitted light is referred to as amplified spontaneous emission (ASE). We measured the ASE spectrum of an SOA manufactured by Thorlabs, model SOA1013SXS [38], and the result is shown in Figure 2.13(a). The FWHM bandwidth was measured to be of $\sim 33 \mathrm{~nm}$ for an injection current of $425 \mathrm{~mA}$. Figure 2.13(b) shows the SOA1013SXS in a fiber-coupled packaged mount with two optical ports (input and output). This model has a low polarization dependent gain (PDG) of $1 \mathrm{~dB}$, being appropriate for amplification in dual-polarization systems.

(a)

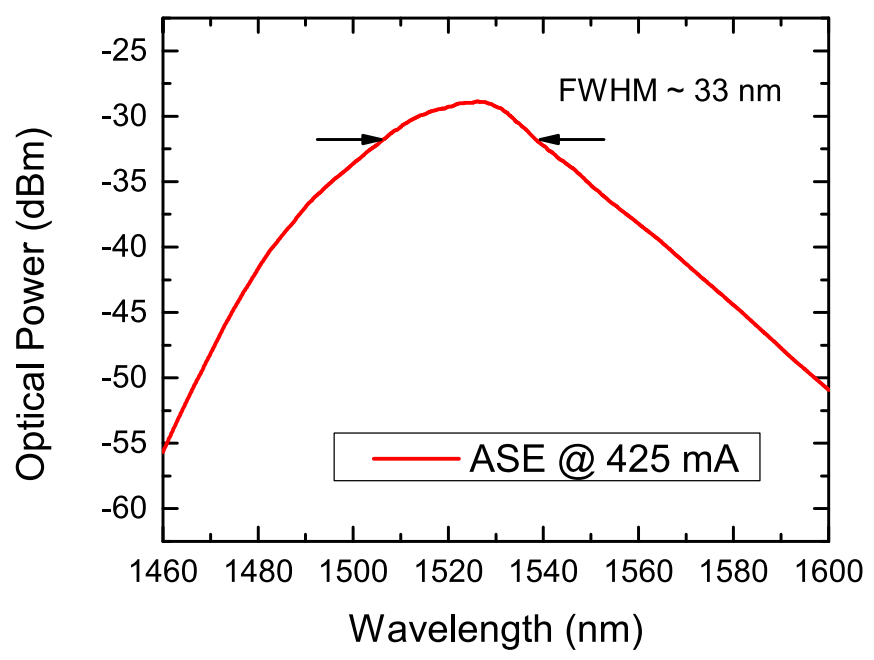

(b)
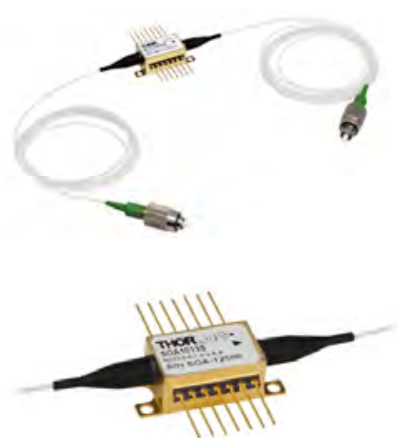

Figure 2.13: (a) Measured amplified spontaneous emission spectrum of SOA1013SXS [38], and (b) its fiber-coupled packaged mount.

As any real optical amplifier, the gain provided by SOAs saturates (see Eq. 2-6). The gain saturation phenomenon can be observed from two equivalent 
angles. For a fixed injection current, the input optical signal experiments linear gain up to a saturation input power $P_{s}$. For input powers higher than $P_{s}$, the gain decreases, and eventually, for even higher input powers, the SOA starts acting as an absorption medium. This behavior can be seen in Figure 2.14, where the gain decreases for high output powers at a fixed injection current of $500 \mathrm{~mA}$. This gain saturation means that at high photon fluxes there will not be enough electrons at the conduction band able to provide gain to all input photons.

Gain vs. Output Power: I = 500 mA

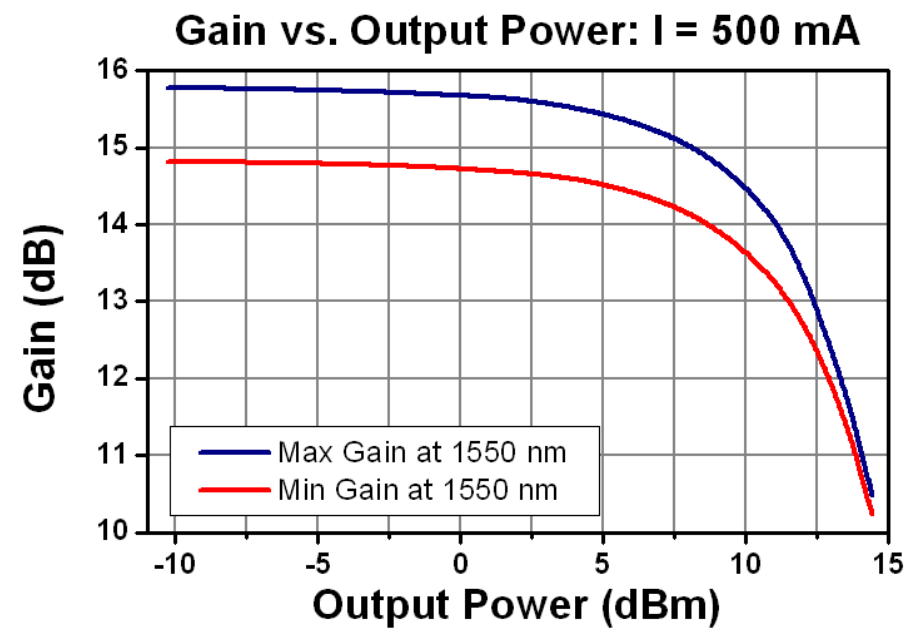

Figure 2.14: SOA1013SXS gain dependence with output power for a fixed driving current of $500 \mathrm{~mA}$ [38].

From another angle, the gain saturation is observed using a fixed input power and varying the injection current. Figure 2.15 shows the measured gain for injection currents varying from 0 to $400 \mathrm{~mA}$. For low SOA driving currents, the population inversion condition is not satisfied, such that the SOA acts as an absorption medium (negative gain). When the current starts to increase, at some point the population inversion condition will be satisfied. Further increases will result in a single pass gain equal to losses (mainly coupling losses and reflection losses), defining a transparency current $I_{T}$. According to Figure 2.15, the transparency current is $\sim 89 \mathrm{~mA}$. For currents higher than $I_{T}$ the SOA provides gain to the input signal, however, further increase in the driving current gives less gain, thus, a gain saturation with driving current is observed. It is important to mention that, for the measurement result shown in Figure 2.15, a low power input signal had to be used, with input power of $-20 \mathrm{dBm}$. Clearly, if a high input power was used, the SOA could act as an absorption medium for any driving current.

Although saturation is an intrinsic property of SOAs, by making use of a time-polarization multiplexing setup we have reported a way to overcome the maximum amplification limit in the pulsed regime [39]. This over-saturation 


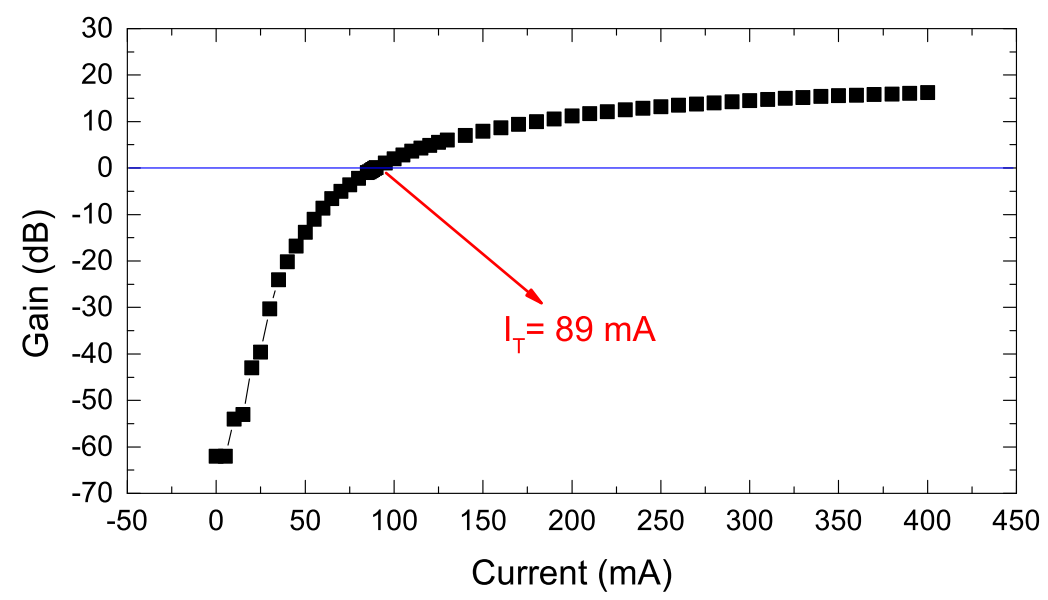

Figure 2.15: Measured SOA gain dependence with driving current.

amplification was only possible in pulsed regime and because of the fast recombination lifetime of carriers in SOAs, which is typically of a few hundreds of picoseconds [37]. A fast recombination lifetime indicates that the device will react quickly to power changes in the input signal, meaning that the output signal will distort if the the operation is close to saturation. This distortion is negligible in fiber-amplifiers, which have much longer recombination lifetimes, usually of the order of milliseconds. Thus, however important for oversaturation amplification in pulsed regime and fast recovery for amplification of a sequence of optical pulses, the fast recombination lifetime of SOAs is often seen as a problem in most optical communication systems, as it adds high frequency noise, increasing the device's noise figure.

Probably the main advantage of SOAs over fiber-amplifiers is its easy integration with optoelectronic devices. For instance, a light source for a WDM system (wavelength division multiplexing) can be built with ten semiconductor lasers and a coupler in the same substrate, where an SOA could be easily integrated at the output to compensate the coupling losses. Thus, however fiber-amplifiers provide higher gains with lower noise, and are less sensitive to polarization of input light, depending on the application, SOAs might be chosen over fiber-amplifiers.

Last, despite we have discussed optical amplification only in a single direction, with one input and one output, actually most optical amplifiers can be used to amplify light in both directions. Clearly, in the case of an SOA, both facets should have anti-reflection coatings. Bidirectional amplification allows optical amplifiers to be used in an optical cavity to provide gain in both directions, thus acting as the gain medium of a laser device. In this case, if an SOA provides a single pass gain $g$, then in a roundtrip a lightwave would experiment a gain of $2 g$. 


\section{3 \\ Rayleigh scattering}

\section{1}

\section{Light scattering phenomena}

Light scattering phenomena are physical processes involving interaction of light with matter. When a light wave impinges in a suspended particle, light is scattered in random directions. Beyond the random change in direction, a change in the optical frequency may also occur. If the scattered light has the same frequency of the incident light, the scattering phenomena is classified as elastic scattering, meaning that there was no energy exchange with the medium. This is a result of Planck's law, which couples the frequency of a light wave with its energy by $E=h \nu$, where $h$ is the Planck's constant. When the scattering phenomenon involves a change in frequency, or energy change, it is said to be an inelastic scattering.

In addition to changes in frequency, the characterization of light scattering phenomena depends both on the properties of incident light, such as wavelength and polarization, and on those of scattering centers, mostly its size and atomic composition. Table 3.1 provides an overview of light scattering phenomena, showing the classification of each phenomenon with regards to scatterer size and energy exchange. Mie scattering was introduced by Gustav Mie in the beginning of the nineteen century, and it is an elastic scattering that takes place when light waves reach particles with size comparable to the wavelength. Mie scattering can be observed in the microscopic water droplets that form the clouds. Compton scattering was introduced later by Arthur Holly Compton in 1923, and it was a remarkable discovery since it cannot be explained by classic wave theory. By analyzing the frequency of scattered light from an incident X-ray in a graphite target, Compton observed a frequency shift proportional to the cosine of the propagation direction of scattered light. This could only be explained with the support of quantum theory, considering light as a stream of photons instead of a wave. When a photon interacts with a charged particle (e.g. an electron), the electron may emit a photon with lower or higher frequency, depending if the photon transmitted energy to the electron, or otherwise, respectively. Raman and Brillouin scatterings are also 


\begin{tabular}{|c|c|c|c|c|}
\hline $\begin{array}{c}\text { Scattering } \\
\text { process }\end{array}$ & $\begin{array}{l}\text { Scattering } \\
\text { origin }\end{array}$ & Size & Energy exchange & $\begin{array}{l}\text { Frequency } \\
\text { shift }(\delta v / v)\end{array}$ \\
\hline $\begin{array}{l}\text { Mie } \\
\text { scattering }\end{array}$ & $\begin{array}{l}\text { particles comparable to the } \\
\text { wavelength - dust, soot, ... }\end{array}$ & $\sim \lambda$ & elastic scattering & 0 \\
\hline $\begin{array}{l}\text { Rayleigh } \\
\text { scattering }\end{array}$ & $\begin{array}{l}\text { particles much smaller than the } \\
\text { wavelength - molecules, } \\
\text { microscopic suspended particles }\end{array}$ & $\ll<$ & elastic scattering & 0 \\
\hline $\begin{array}{l}\text { Raman } \\
\text { scattering }\end{array}$ & $\begin{array}{l}\text { vibration and/or rotational } \\
\text { mode of a molecule - optical } \\
\text { phonon }\end{array}$ & $*$ & inelastic scattering & $\pm\left(10^{-5}-10^{-1}\right)$ \\
\hline $\begin{array}{l}\text { Brillouin } \\
\text { scattering }\end{array}$ & $\begin{array}{l}\text { acoustic mode of molecules - } \\
\text { acoustic phonon }\end{array}$ & * & inelastic scattering & $\pm\left(10^{-6}-10^{-5}\right)$ \\
\hline $\begin{array}{l}\text { Compton } \\
\text { scattering }\end{array}$ & charged particles - electrons & $\ll \lambda$ & inelastic scattering & $\pm\left(10^{-3}-10^{-2}\right)$ \\
\hline
\end{tabular}

Table 3.1: Comparison of light scattering phenomena [40].

inelastic scatterings, which can take place in liquids, gases or solids. These are particularly interesting as they can be observed in optical fibers. Both are related to variations in the states (modes) of molecules in a medium, Raman scattering being associated with vibrational modes, assuming higher frequency shifts, whereas Brillouin scattering are related to acoustic modes, having lower frequency shifts. In solids, e.g. in standard optical fibers, Raman and Brillouin scatterings involve the presence of optical phonons and acoustic phonons, respectively. Rayleigh scattering was introduced by Lord Rayleigh in the late nineteen century, and it an elastic scattering given by particles with size much smaller than the wavelength. This phenomenon is of uttermost importance in this thesis, and the following section is dedicated to a thorough analysis and modeling of Rayleigh scattering in single mode fibers.

\section{2}

\section{Rayleigh backscattering in optical fibers}

Rayleigh backscattering in single-mode optical fibers is a phenomenon of great interest for many research areas, such as optical-fiber sensing [41], bidirectional transmission systems with optical fibers [42] and random distributed feedback fiber lasers [1]. The Rayleigh scattering phenomenon occurs when light reaches particles with dimensions much smaller than its wavelength $(\lambda)$, and light is scattered in all directions. Rayleigh scattering can be observed in many backgrounds, for example, in the atmosphere, in porous materials, 
fluid suspensions and in optical fibers. In optical fibers, part of the Rayleigh scattered light couples in the backward direction and is guided back to the input end. The intensity of backscattered light, composed of the sum of the contribution of all scattering centers throughout the fiber, is a stable quantity provided that an incoherent light source is used. In case of a coherent light source, the backscattered light exhibits strong random intensity fluctuations, often referred to as Coherent Rayleigh noise (CRN) in OTDR measurements [4], which are due to the interference of light backscattered at different portions of the fiber within the coherence length of the light source.

Although several techniques have been employed to mitigate coherent Rayleigh noise in different applications [43-46], $\Phi$-OTDR technology makes use of light interference within the OTDR pulse width to detect vibrations, acoustic waves and temperature variations with high sensitivity [47]. Thus, either to mitigate or take advantage of interference of Rayleigh backscattered coherent light, it is important to quantify the phenomenon before designing optical systems that may be affected by the phenomenon. In the following section we provide a review of some of the most relevant models for Rayleigh backscattering in single mode fibers.

\subsection{1}

\section{Review of Rayleigh backscattering models}

The modeling of Rayleigh backscattering in optical fibers has been approached in many works, often targeting fiber monitoring applications like OTDR and $\Phi-O T D R$ [24-29]. Nakazawa developed a theory for backscattering in single mode fibers and derived a simple formula for the total backscattered power [24]. The theory was built for OTDR applications, which considered a pulsed light source. The backscattered power formula was derived based on two models, Booker-Gordon and Gaussian, both using an average refractive index along the fiber. Although the theory can be employed to accurately calculate the total backscattered power, it cannot be used to predict the backscattered intensity fluctuations since it does not consider neither a coherent light source, nor a fluctuating refractive index.

A number of works reported analysis of the intensity fluctuations and statistical properties of Rayleigh backscattering of coherent light in optical fibers [25-27,48]. Eickhoff was one of the first to publish experimental measurements of such intensity fluctuations [48]. The experiment considered a short fiber of $17 \mathrm{~m}$, and a coherent light source. Intensity fluctuations were associated with a variation of the propagation constant in time, which varies with environmental changes (temperature and vibration), and with laser frequency drift. 
It was reported that the statistics of the backscattered intensity presents an exponential distribution. In a later work, aiming to analyze the statistics of Rayleigh backscattered light for OTDR applications, Healey modeled the amplitude of backscattered light as a random linear filter, and derived its impulse response [27]. It was assumed that the amplitude of backscattered signal is a random variable. From the convolution of the impulse response with an input light pulse of random phase, the probability density function of backscattered signal was derived. It was shown that the backscattered light becomes less random for sources with coherence lengths shorter than the OTDR pulse width, while for longer ones the intensity distribution is exponential. In other works [25, 26], P. Healey showed that the field amplitude of Rayleigh backscattering has a Rayleigh distribution, which is equivalent to an exponential distribution of intensity fluctuations, as the square of a Rayleigh random variable is an exponential random variable. The results of Healey's works are useful for coherent-OTDR applications and coincide with experimental measurements, but the papers do not discuss the origin of the randomness of its random linear filter, neither the fluctuation of the refractive index.

Staubli and Gysel reported a model for Rayleigh backscattering that indeed considers refractive index fluctuations [49]. They partitioned an optical fiber into a discrete number scattering sections with length much longer than the correlation length of refractive index fluctuations. Each section contributed independently to the total backscattered field, which was obtained by summing over every section. In such a way, the randomness of the process was fully attributed to the source, which was modeled with random amplitude and phase. A remarkable similarity was shown between theoretical model and experimental measurements, although it only considered low coherence sources. Despite the coincidence with real measurements, the theory does not model truly Rayleigh backscattering, as their scattering sections can extend up to a few micrometers, which rather falls in the Mie scattering range. Also, although the work was specific for low coherence sources, if high coherence ones were considered, index fluctuations in a larger spatial scale could not be neglected, and a deviation between model predictions and experimental measurements would probably be observed.

A model that considers both macro $(\gg \lambda)$ and sub-micro $(\ll \lambda)$ scale physics is described in [29]. For the macro scale aspect, it was argued that the refractive index varies with position for intervals much longer than $\lambda$, however no physical reason was discussed in detail. Since the paper targets OTDR applications, where small portions of the fiber are evaluated independently (within the OTDR pulse width), the macro scale index fluctuations were neglected 
in the final model. The modeling of sub-micro scale features was performed through the definition of a discrete set of scattering centers, associated with microscopic density fluctuations. Each center has a random scattering amplitude and is located at a random position within a fixed interval, such that every interval includes a single scattering center. Also, the average distance between two scattering centers was said to be much greater than the wavelength. Although authors carefully argued that each element of the discrete set of scattering centers can be understood as an aggregate of multiple neighboring scatterers, this modeling approach suggests that subsequent scatterers contribute with a highly different phase because they are far apart. However, this proposal is unreal because the scattering occurs in nanometric scale, such that subsequent scatterers contribute with approximately the same phase.

An interesting model, in the context of $\Phi$-OTDR, was proposed by Zhou et al. in [28]. Authors comment that the large number of scatterers makes the backscattering highly homogeneous over the fiber length, which cannot explain the intensity fluctuations. Since the polarization fading was shown not to be sufficient for the measured intensity fluctuations, it was suggested that a macro scale fluctuation of the index should be responsible for the phenomenon. Thus, a position-dependent refractive index was assumed in their model, with index fluctuations related to different temperatures and strains along the fiber. It was also considered that scatterers have random amplitudes, governed by a Gaussian distribution. Unknowing the index position dependence, authors modeled index variations as a sine function with small amplitude and a period varying from 4 to $10 \mathrm{~m}$. Although it was shown that amplitudes as small as $2 \times 10^{-6}$ were enough to cause intensity fluctuations very similar to those observed in the experiments, there is no physical basis to assign a sinusoidal function for the refractive index. It is possible that the agreement with experimental measurements was due to an analysis restricted to short fiber sections, as the simulations considered OTDR pulses with temporal widths up to $50 \mathrm{~ns}$.

As the Rayleigh backscattered intensity fluctuates in time, it can be classified as a random dynamical system. The statistical properties of dynamical systems are often analyzed with the aid of ergodic theory. The fundamental objective of ergodic theory is to study the long-term statistical behavior of dynamical systems [50]. This theory gave birth to the concept of ergodic process: if the statistical properties of a stochastic process $x(t)$ can be derived from a single and long enough random sample of $x(t)$, then the process is said to be ergodic [51]. An interesting property of ergodic processes is that they may present the same mean averaged over the time-space, and over the 
system's entire possible states space, i.e., the average of the system in time domain equals to that of several replicas of the system at a given time [52]. This property is often referred to as ergodicity [53]. For instance, Poole claims that polarization mode dispersion (PMD) in optical fibers exhibits ergodicity [30], and this property is actually used in all PMD measurement techniques [54]. For low coherent sources, Staubli and Gysel [49] mathematically showed that the field amplitude of Rayleigh backscattered light is an ergodic process, in the sense that the average of backscattered amplitudes derived from many similar fibers at a given time is identical to that of a single fiber averaged over a long time period. Although this is an interesting mathematical result, it is unpractical for real applications, not only because it considers only low coherence sources but also because a representative measurement of amplitude fluctuations for a single fiber takes a long time, and an average over multiple fibers is unreasonable.

Considering all the rather different viewpoints and modeling approaches discussed above, as well as conflicting and misleading ideas on the origin of the scattering, a thorough analysis of the phenomenon of Rayleigh backscattering of light with any coherence length in optical fibers will be helpful in understanding many experimental phenomena and applications. In the next section, a thorough modeling of Rayleigh backscattering of coherent light is proposed considering both nanometric and long-range index fluctuations. We provide physical and simulation evidences that index variations as small as $10^{-7}$ are sufficient to cause backscattered intensity fluctuations, where no randomness needs to be attributed to the scatterers' amplitude.

\subsection{2}

\section{Theoretical model for Rayleigh backscattering}

The origin of classical Rayleigh scattering comes from molecules that form a radiating dipole when in the presence of an electric field. Molecules exhibiting a higher tendency to acquire an electric dipole moment, i.e., molecules with higher polarizability, produce stronger radiations. This classical explanation was given in the iconic paper of Lord Rayleigh [55], where he explained the origins of the blue sky. Currently, Rayleigh scattering has a broader sense, encompassing the scattering from any volume (cluster of molecules) with size much smaller than the wavelength of light [56]. Thus, any small particle suspended in a medium of different refractive index is treated as a scattering center. However, in optical fibers, the interpretation of Rayleigh scattering is slightly different, and it is explained as follows.

Standard single mode optical fibers are composed of Silica and Germania 
molecules, which define a vitreous structure. Different than crystals, which are characterized by an ordered arrangement of molecules, vitreous structures have molecules randomly arranged, configuring an amorphous medium. However, it has been shown that silica glasses are not entirely disordered, exhibiting an intermediate-range order that extends up to about $5 \mathrm{~nm}[57,58]$. The range order defines a correlation length $l_{c}$, within which molecules are nearly ordered, thus forming a region with constant density. For distances longer than the correlation length, molecules lose their order and are arranged in a different fashion, which can be more densely organized or not. Figure 3.1(a) shows a schematic in two-dimensions of the structure of a pure Silica fiber, where a transition between two nearly ordered regions of different densities is represented.

From an optical viewpoint, a fluctuation in density translates into a fluctuation of the refractive index. Thus, given the amorphous structure of optical fibers, its refractive index fluctuates in a small spatial scale. Figure 3.1(b) shows a representation of a small section $(\ll \mu \mathrm{m})$ of an optical fiber, where regions with nearly ordered molecules are identified with black lines for eyes guidance. Atoms are represented with orange dots, and their quantity indicates the density of a given region. Whenever a light wave reaches a volume with different refractive index, which occurs multiple times in a structure as shown in Figure 3.1(b), light is scattered in all directions. Note that, the higher is the fluctuation between adjacent volumes, the stronger is the scattering. As the correlation length of Silica glasses is of the order of a few nanometers, the regions defined in Figure 3.1(b) are much smaller than the wavelength of light, thus the light scattering phenomenon is classified as Rayleigh.

Although a fraction of incident light is scattered due to small index fluctuations, most of it is transmitted. The phenomenon of light propagation in an amorphous optical fiber can be described as follows. Let us write the refractive index of an arbitrary volume element $m$ with constant refractive index as an average refractive index $\bar{n}$, added by a random deviation $\delta n_{m}$ with zero mean, i.e.,

$$
n_{m}=\bar{n}+\delta n_{m} .
$$

The average refractive index is the mean value over the fiber length, while the index deviation defines an increment or reduction over the average index. From Eq. 3-1, it is clear that the refractive index of the medium is a position-dependent quantity, which will be written henceforth as $n(z)$, with index deviations also written as a function of position, $\delta n(z)$. Neglecting the time dependence, to calculate the phase propagation through a fiber section 


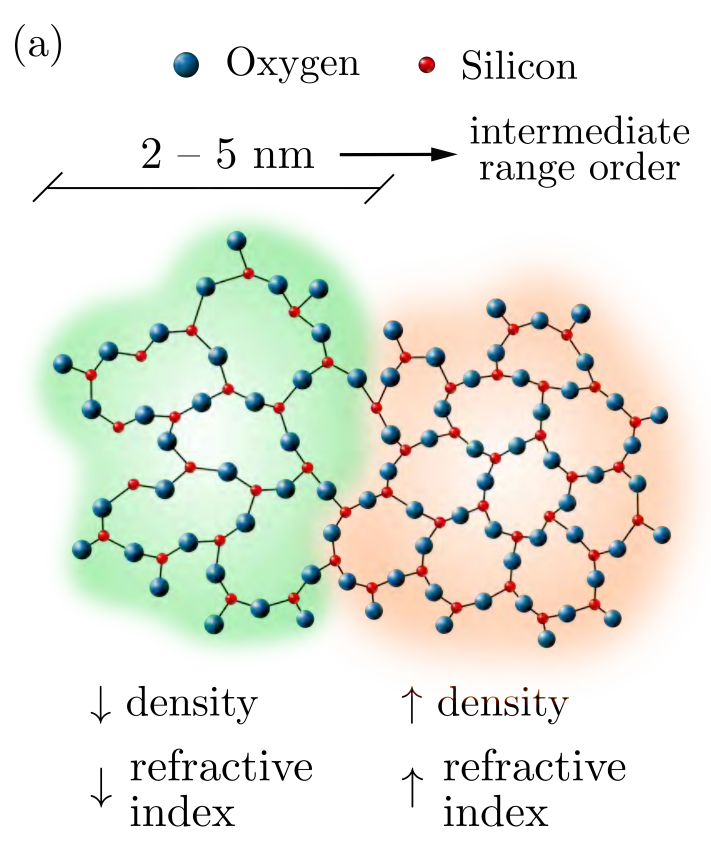

(b)
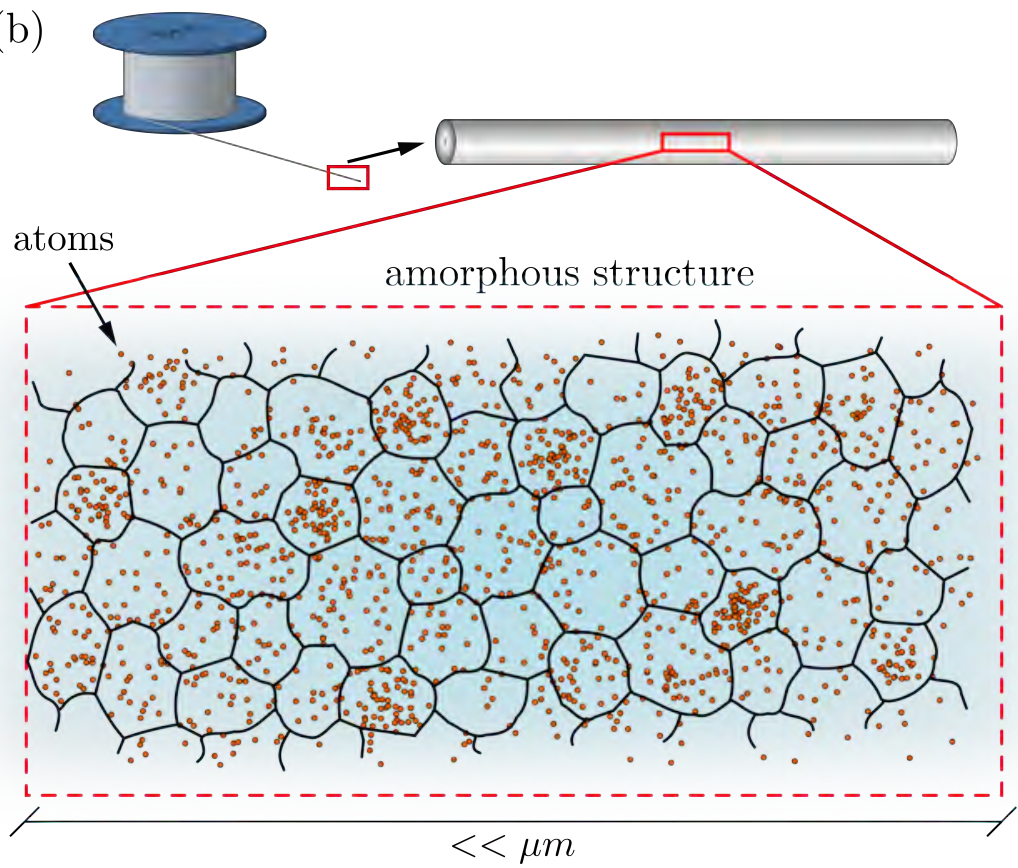

Figure 3.1: Schematic representation of the amorphous structure of optical fibers. (a) The arrangement of molecules in silica fibers exhibit an intermediate range order, defining volume elements of constant densities. The transition between two nearly ordered regions is indicated as the border between green and orange regions, the latter encompassing molecules more densely arranged, defining a region with greater refractive index. (b) Schematic of a fiber section in sub-micron scale. Atoms are represented by orange dots, and black lines define regions of constant density.

of arbitrary length $z$ we must perform the following integration:

$$
\phi(z)=\int_{0}^{z} n\left(z^{\prime}\right) k d z^{\prime}=\int_{0}^{z}\left(\bar{n}+\delta n\left(z^{\prime}\right)\right) k d z^{\prime}=\bar{n} k z+\left[\int_{0}^{z} \delta n\left(z^{\prime}\right) d z^{\prime}\right] k,
$$


where $\mathrm{k}$ is the vacuum propagation constant.

Since $\delta n\left(z^{\prime}\right)$ is a random variable with zero mean, the term inside square brackets in Eq. 3-2 vanishes, resulting in a phase propagation governed by the average refractive index. Thus, from the perspective of light propagation, such medium is called optically homogeneous [59]. This is compatible with fundamental electromagnetic theory of light propagation in optical fibers, which approximates light beams as propagating plane waves in the fiber's core. Indeed, would these short-range index fluctuations be high enough to impart a relevant phase shift, transverse fluctuations of the refractive index along the fiber core diameter $(\sim 10 \mu \mathrm{m})$ would randomize the propagating phase, violating the primary principles of light propagation in optical fibers.

For the purpose of modeling Rayleigh backscattering, it should be clear by now that light is scattered continuously along the fiber, such that neighboring scatterers contribute to the overall backscattered signal with approximately the same phase. Similarly, as there is a large number of scatterers for any transverse section of the fiber, it is assumed that light is scattered with the same intensity at each position $z$, which is represented by an average backscattering field coefficient $C$. In this way, we start our model considering the Rayleigh backscattered light generated in a single mode fiber by a monochromatic CW laser. To simplify the approach, we neglect fiber birefringence and polarization effects. The amplitude of the total backscattered optical field $E_{b}(z)$ at the launching point $z=0$ is given by the coherent sum of the contributions of all sections of the fiber back propagated to the origin, which can be written as

$$
E_{b}(z=0, k)=E_{0} C A \int_{z=0}^{L} \mathrm{e}^{-\alpha z} \mathrm{e}^{-j \bar{n} k z} d z,
$$

where $E_{0}$ is the launched field, $\alpha$ is the power attenuation coefficient of the fiber and $A$ is the mode field effective area. Note that, instead of using a summation over every scattering center in Eq. 3-3, an integral was employed, providing better representation of the continuous scattering along the fiber. The solution of Eq. 3-3 is analytic, giving a backscattered optical field proportional to $\operatorname{sinc}(\bar{n} k L)$, thus inversely proportional to the fiber length and phase varying with the full roundtrip fiber length. This means that in the same way as in a uniform crystal, no backscattered coherent light would be observed, which is obviously wrong: Eq. 3-3 must be corrected.

Even though we argued that an average refractive index should be considered in Eq. 3-3, this would not explain the backscattering intensity fading at the input end $[12,28]$. This phenomenon is only explained when index fluctuations are considered in a scale much larger than the wavelength. 
Thus, we investigate the range of index fluctuations caused by residual stress in optical fibers. Residual stress, often referred to as frozen-in stress, is that present even without externally applied forces. Given the importance of residual stress in optical fibers, which, for instance, influences the fiber's refractive index and its birefringence [60], many works reported studies on this topic, and an extensive review is presented in [61]. The causes of residual stress can be divided into two major groups: thermal and mechanical. The thermal expansion coefficient of glasses depends on its composition and on the arrangement of molecules at the fictive temperature. As shown in Figure 3.1, the random arrangement of molecules in glasses is given in a sub-micron scale. Thus, thermal stresses cannot be the cause of index fluctuations in a scale much larger than the wavelength. On the other hand, mechanical stresses are generated during the fiber manufacturing process, leading to variations of the refractive index that can extend to larger scales.

The drawing tension applied to optical fibers during the manufacturing process is the direct cause of mechanical stresses. When higher drawing tensions are applied, the greater is the impact on the refractive index. In [61], it was shown that a linear relation exists between the change in the refractive index and the drawing tension applied, where a slope coefficient of $5 \times 10^{-6} / \mathrm{g}$ was found, and index changes as large as $1 \times 10^{-3}$ were measured for a drawing tension of $200 \mathrm{~g}$. This tension is proportional to the speed at which a capstan pulley rotates, pulling optical fiber in the process. The capstan speed is controlled to maintain the fiber diameter close to $125 \mu \mathrm{m}$, where a tolerance of $\pm 0.1 \mu \mathrm{m}$ is accepted in most specification designs [62]. The adjustment of the capstan speed is performed by a loop control system, which is composed mainly of a diameter monitor (input) and a controller device that acts on the capstan's speed (output). According to [62], the capstan speed is updated at rates varying from 1 to $20 \mathrm{kHz}$. Thus, considering an update rate of $5 \mathrm{kHz}$ and a normal capstan speed of $50 \mathrm{~m} / \mathrm{s}$, then the drawing speed is updated for every centimeter of pulled optical fiber. Hence, tiny random variations in the controlled speed, which arise from many factors - noise from diameter monitor, imprecision of torque and speed motors, disturbances in the loop controller would result in random residual stress for fiber sections approximately $1 \mathrm{~cm}$ long. Assuming that the controller step is ten times better than the tolerable design goal $( \pm 0.01 \mu \mathrm{m})$, then a fluctuation of the capstan speed (or drawing tension) might be of the order of $0.01 \%$. Therefore, considering a change in the refractive index of $5 \times 10^{-6}$ per gram of drawing tension, and a normal drawing tension of $200 \mathrm{~g}$, then the random stresses cause a random variation of the refractive index of the order of $10^{-7}$ for every centimeter of optical fiber. 
With residual stress randomly modulating the refractive index in a centimetric scale, then the effective refractive index for light propagation purpose should be written as

$$
n(z)=\bar{n}+\Delta n(z),
$$

where $\Delta n(z)$ is a random variable with zero mean, representing index changes due to residual stress. Note that the nanometric scale fluctuation of the refractive index, $\delta n$, is the key element to explain the Rayleigh scattering in optical fibers, whereas the centimetric scale fluctuation $\Delta n$, is responsible for the backscattered intensity fading. Over fiber sections smaller than a centimeter, yet much larger than $l_{c}$, a local average refractive index can be considered for light propagation purpose. This local average index fluctuates for sections longer than a centimeter. In such a way, it is convenient to divide a fiber with length $L$ into $N$ sections of length $d$, where $d \sim 1 \mathrm{~cm}$. To each of these sections is attributed a local average refractive index $\bar{n}_{i}$, where $i$ is an integer belonging to the interval $[1, N]$. The local average indexes are modeled initially by $\bar{n}_{i}=1.44+\xi_{i} 10^{-7}$, where $\xi_{i}$ is a uniformly distributed random number in the interval $[0,1]$. By assuming constant refractive indexes for sections with length $\mathrm{d}$, the backscattered optical field can be piecewise calculated, performing analytical integration along these sections, and summing over all sections. Thus, the backscattered optical field is now written as

$$
\begin{gathered}
E_{b}(z=0, k)=E_{0} C A \sum_{i=1}^{N} E_{i} \\
\text { where } E_{i}=\exp \left(j 2 \phi_{i-1}\right) \int_{-d / 2}^{d / 2} \exp \left(-\alpha\left(l+z_{i}\right)-j 2 \bar{n}_{i} k l\right) d l \\
\text { and } \phi_{i}=\phi_{i-1}+\bar{n}_{i} k d .
\end{gathered}
$$

The model expressed by Eq. 3-5 is represented in Figure 3.2. To calculate the field contribution $E_{i}$ of the $i^{\text {th }}$ fiber section centered at position $z_{i}$ with refractive index $\bar{n}_{i}$, one should determine the accumulated phase immediately before such section, represented by $\phi_{i-1}$. The factor 2 multiplying this accumulated phase term represents the roundtrip to the input position. Without loss of generality, it can be considered an input phase $\phi_{0}=0$, allowing the iterative calculation of $E_{b}(z=0, k)$. Also, note that Fresnel reflections at index mismatched positions are neglected because of their minimal contributions, hence Eq. 3-5 models only Rayleigh backscattering. Last, the backscattered intensity of Rayleigh light is calculated by taking the absolute square value of Eq. 3-5.

The prediction of the backscattered intensity at the input end of the fiber 


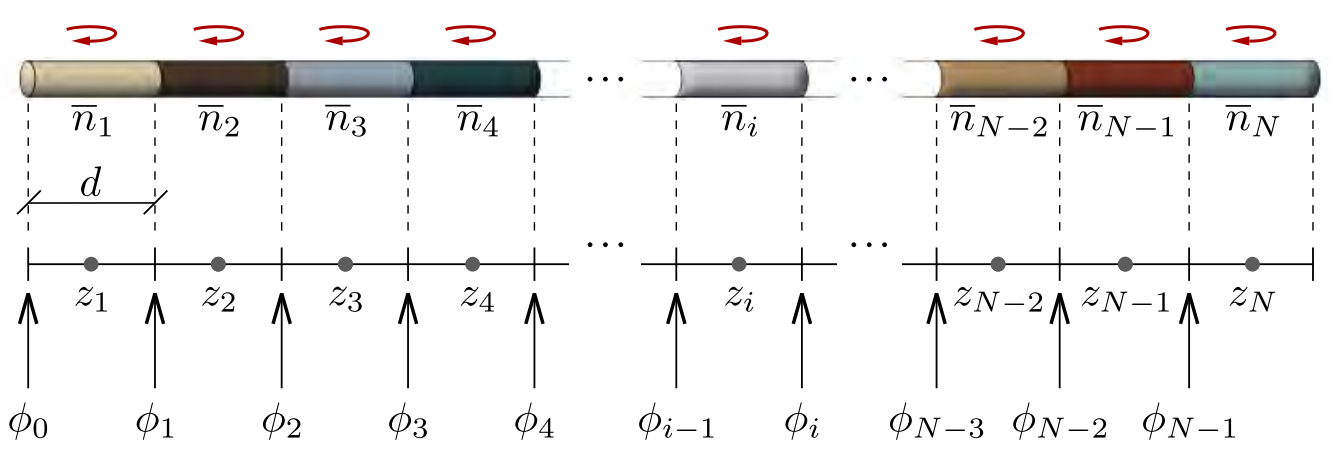

Figure 3.2: Representation of theoretical model for Rayleigh backscattering intensity fluctuations in single mode fibers. Given the nanometric scale refractive index fluctuations, it is assumed a continuous scattering along sections of length $d$ ( $\sim 1$-cm long), with a local average refractive index $\bar{n}_{i}$ for an $i^{\text {th }}$ section. The centimetric scale index fluctuations, caused by residual stress, results in a different local average index for each section $d$.

is thus calculated independently for each frequency (or wavenumber), such that a prediction over a large frequency bandwidth is straightforward. Compared with previously reported models, the one presented in this work is relevant for the reasons stated below:

1. It is established over fundamental physical properties, considering both sub-micro and macro scale aspects. Solid reasoning behind index fluctuations in both scales is provided, clarifying misleading interpretations of the phenomenon in previously reported models.

2. For the first time, Rayleigh backscattering intensity fluctuations are modeled in frequency domain, eliminating any assumptions regarding the behavior of environmental changes along the optical fiber, or even regarding laser amplitude/phase fluctuations.

3. Model predictions in frequency domain can be used to obtain timedomain statistical behavior of Rayleigh backscattering properties in single mode fibers.

4. As will be further clear, the model is valid for fibers of any length and for light sources of any linewidth, thus being an important tool in Rayleigh backscattering based random fiber lasers as well as in $\Phi$-OTDR applications. 


\subsection{3}

\section{Simulation results}

The theoretical model described in previous section was used to simulate the intensity fluctuations of Rayleigh backscattering for three different fiber lengths: 10, 100 and 1,000 m. For probability calculations and statistical modeling, the mode field effective area $A$, the field backscattering coefficient $C$ and the input optical field $E_{0}$ are irrelevant because only normalized quantities are compared. The optical power attenuation coefficient was set to $0.2 \mathrm{~dB} / \mathrm{km}$, and the backscattered intensity was calculated for a set of propagation constants $k$, corresponding to a frequency bandwidth of $20 \mathrm{MHz}$ (centered at $\nu=194 \mathrm{THz}$ ). For numerical simulation purpose, we used frequency increments $f_{i}$ of $250 \mathrm{~Hz}$ (sampling interval), so to calculate the backscattered intensity for a bandwidth of $20 \mathrm{MHz}$, we performed 80,000 calculations of Eq. 35. To minimize windowing effects arising from a fixed $d=1 \mathrm{~cm}$, simulated optical fibers were generated as a sequence of fiber sections with uniformly distributed random lengths, between 0.95 and $1.05 \mathrm{~cm}$ - each section having a random refractive index between 1.4400000 and 1.4400001. Simulation results are presented in Figure 3.3(a).

According to Figure 3.3(a), intensity fluctuations of longer fibers present stronger frequency dependence than shorter ones. This behavior agrees with Eq. 3-5, as the randomness present in the refractive index term is magnified by the fiber length, and because more random contributions are considered. Note that the mean intensity growth with fiber length is simply caused by more light being scattered in longer fibers.

The curves plotted in Figure 3.3(a) indicate that longer fibers require better frequency resolutions, or smaller frequency increments $f_{i}$, to represent intensity fluctuations without aliasing effects. It is thus necessary to verify if the selected $f_{i}$ is adequate to simulate intensity fluctuations for the fiber lengths chosen. Beyond avoiding aliasing effects, the definition of a relationship between minimum frequency increment and fiber length is important to reduce computational processing time during simulations.

We investigated this issue by extending the simulations for fiber lengths up to $50 \mathrm{~km}$, again using $f_{i}=250 \mathrm{~Hz}$ within the spectral width of $20 \mathrm{MHz}$. The fast Fourier transform (FFT) of simulated field fluctuations is presented in Figure 3.3(b), where longer fibers exhibit wider spectra, but all limited by a cut-off spectral component. The cut-off spectral component determines the Nyquist limit for the smallest frequency increment that can be used in the simulation and still providing a good representation of $E_{b}(0, k)$ for a given fiber length. The field and intensity fluctuations of the longest fiber could be 

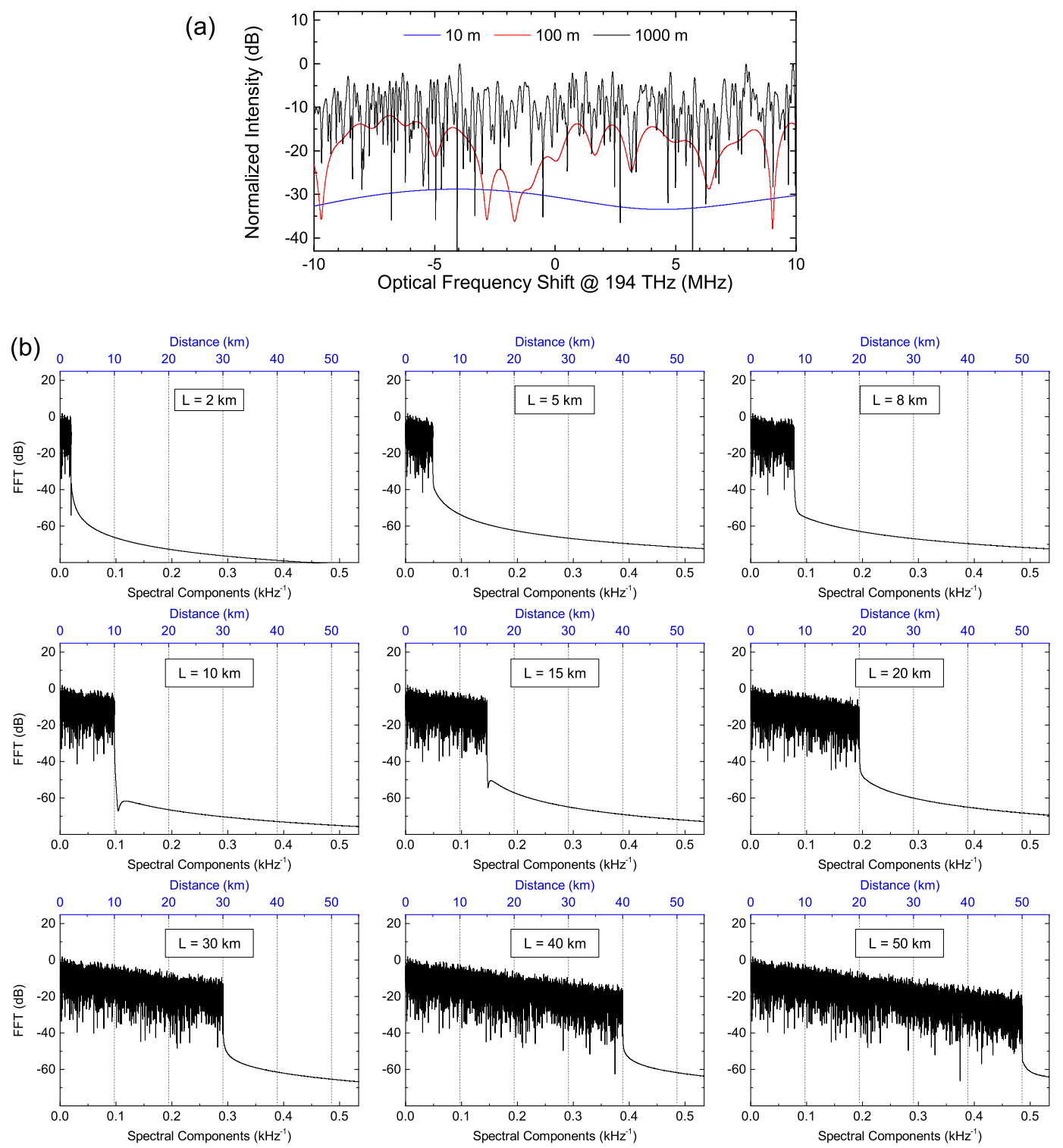

Figure 3.3: (a) Simulated intensity of Rayleigh backscattered light for three fiber lengths. (b) FFT results of simulated optical field fluctuations for different fiber lengths.

easily reproduced by the selected frequency increment. In fact, according to Figure 3.3(b), using frequency increments of $1 \mathrm{kHz}$ (Nyquist limit $=0.5 \mathrm{kHz}^{-1}$ ) would still reproduce well the fluctuations for a $50 \mathrm{~km}$-long fiber, with a fourfold reduction in the simulation time. The same configuration used to generate the results in Figure 3.3(b) was repeated for new sets of randomly generated fibers. Results showed that different fiber samples generated with the same length present the same cutoff and envelope spectrum, so that the traces in Figure 3.3(b) represent a general case scenario.

The spectra in Figure 3.3(b) are actually very similar to Optical Frequency Domain Reflectometry (OFDR) traces [63] except that in OFDR there is a reference signal beating with the backscattered light, and the FFT is ob- 
tained from the intensity vs frequency curve. Here, no reference signal is provided, and the interference occurs between the light scattered along the full fiber length. The FFT is taken from the optical field frequency dependence, the same procedure used in the subcarrier tone sweeping method used for fiber monitoring in Radio over Fiber links [64], except that here it is the frequency of the optical carrier which is swept instead of a subcarrier modulation frequency. Indeed, the upper axis scale was included to display the FFT power spectrum in spatial units by multiplying the lower scale by the velocity of the light in the fiber and dividing by a factor of two to account for the round-trip path. The spectral power decays with $\sim 0.2 \mathrm{~dB} / \mathrm{km}$, and presents a sharp cutoff at the fiber end, as an OTDR trace. The spectra in Figure 3.3(b) clearly show that there is a correspondence between length and FFT spectral power, and even faults along a fiber could be measured by this method as in [64].

The model simulation of the OTDR trace can then be used to predict and evaluate the sensitivity of $\Phi$-OTDR measurements to local temperature and/or stress variations. Figure 3.4(a) displays the simulated OTDR trace of a 1-km fiber obtained using $200 \mathrm{MHz}$ optical bandwidth, which corresponds to a sampling density of $2 \mathrm{~m}^{-1}$ in the OTDR trace. The temperature of a $10 \mathrm{~m}$ long section located at $200 \mathrm{~m}$ from the origin was varied by $0.1{ }^{\circ} \mathrm{C}$ in ten steps by adding a mean index variation of $1.15 \times 10^{-5} /{ }^{\circ} \mathrm{C}$ [65] to the fiber section between 200 and 210 m. The simulated results, zoomed in Figure 3.4(b), clearly show the sensitivity of the measurement technique and the power of the model in predicting the effect of local variations of environmental conditions on the fiber properties. Even though the simulation here was performed for a full fiber, it is straightforward to see that the model can be used to evaluate different experimental conditions such as pulse duration, sampling resolution or coherence length of the source in $\Phi$-OTDR measurements.

Polarization effects in OFDR are important and indeed observable [63] because the polarization of the reference optical field is constant, so that every rotation in the backscattered polarization from a given fiber position will induce fading in the detected signal. In the present case, however, birefringence effects will only add to the local refractive index fluctuations a further random variable so that they are averaged out in the model.

We analyzed the impacts on $\Phi$-OTDR traces in case residual stress fluctuations manifest at different spatial periods. A new simulation was performed, where seven fiber events with the same length $L=1,000 \mathrm{~m}$ were numerically generated by defining fiber sections $d$ with lengths ranging from $0.001 \mathrm{~m}$ to 1,000 $\mathrm{m}$. The simulation optical frequency bandwidth was kept at $200 \mathrm{MHz}$. In each of the seven cases, the refractive index remained constant throughout 


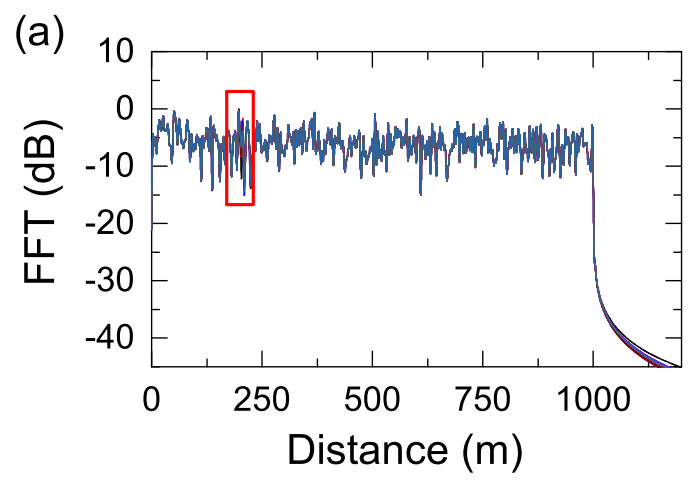

(b)

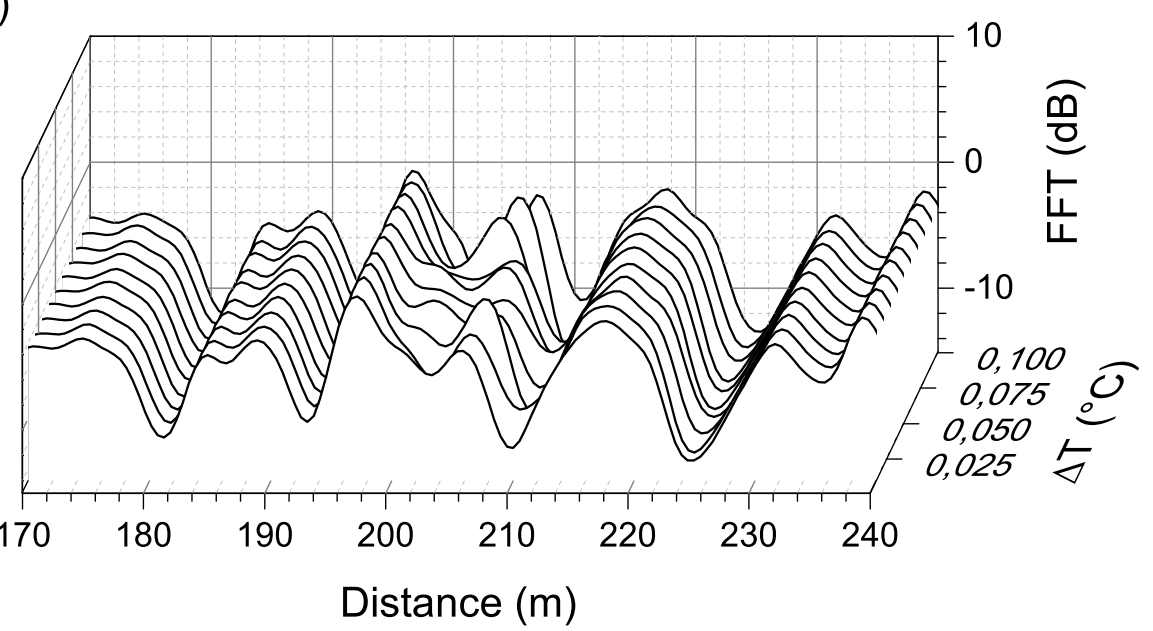

Figure 3.4: (a) Simulated phase-OTDR trace for a 1-km long fiber with temperature varying up to $0.1{ }^{\circ} \mathrm{C}$ at position $z=200-210 \mathrm{~m}$. (b) Zoom in the region of interest.

$d$, still complying with the theoretical model summarized in Figure 3.2. Note that, the spatial periodicity $d$ is directly related to the fiber manufacturing process. In theory, if during the manufacturing process the control loop update rate was lowered, then the sections $d$ would get longer. And if the loop control was completely turned off, then the fiber section $d$ would correspond to the total fiber length $L$. Simulation results are shown in Figure 3.5.

According to results shown in Figure 3.5, the periodicity of residual stress has a significant impact on the $\Phi$-OTDR traces. As the fiber sections of constant refractive index $d$ get longer, their periodicity starts to appear at the $\Phi$-OTDR trace. This behavior starts being observed at $d=10 \mathrm{~m}$, where a zoom in shows a $10 \mathrm{~m}$ period clearly observed in the trace. At $d=100$ and $1,000 \mathrm{~m}$, narrow peaks arise at the exact positions where the refractive index changes randomly in the seventh decimal place. Since the $200 \mathrm{MHz}$ simulation bandwidth allows a numerical sampling of 2 points per meter, we could not observe a clear period for sections $d$ less than $1 \mathrm{~m}$. However, results clearly 


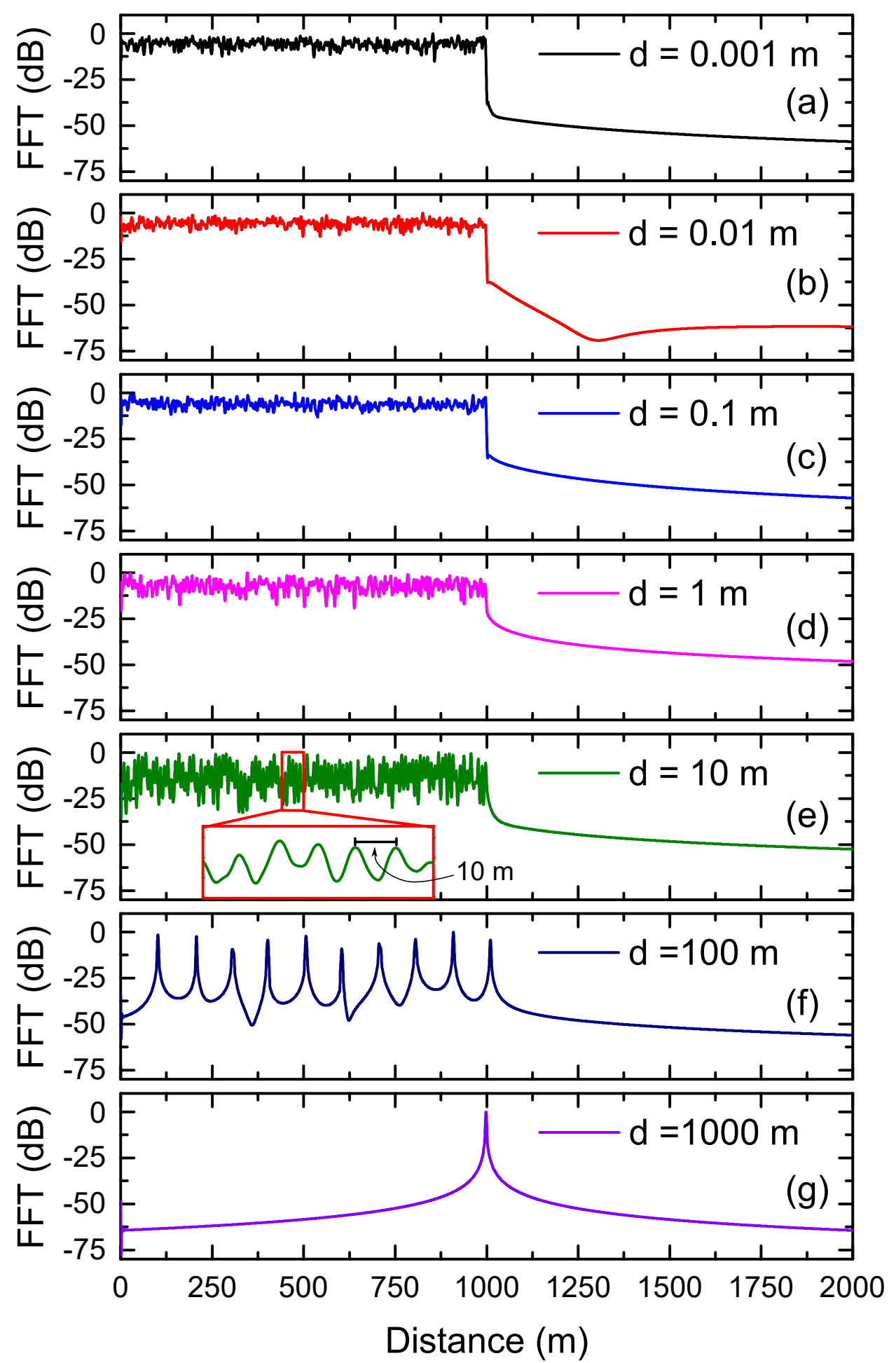

Figure 3.5: Simulation results for phase-OTDR traces considering residual stress imposed at multiple spatial periods, ranging from $0.001 \mathrm{~m}$ to $1,000 \mathrm{~m}$. Amplitudes were normalized by the maximum value.

indicate a limitation of $\Phi$-OTDR measurements, which can have resolutions as good as the refractive index macro scale spatial fluctuation.

The probability density functions (PDF) of simulated intensity fluctuations are provided in Figure 3.6(a), with intensities normalized by the mean 
value. A single fiber length of $8 \mathrm{~km}$ was assessed for three different bandwidths: $1 \mathrm{MHz}, 20 \mathrm{MHz}$ and $1 \mathrm{GHz}$. Although the simulations with a bandwidth of $20 \mathrm{MHz}$ already provided the same spectral components present in $1 \mathrm{GHz}$ bandwidth, larger bandwidths were required to visit extreme intensity events. This behavior is evident in Figure 3.6(a), where intensity fluctuations almost 14 times greater than the mean value were observed for a $1 \mathrm{GHz}$ bandwidth, while fluctuations only nearly 5 times greater than the mean value were present in a $1 \mathrm{MHz}$ bandwidth. Despite this difference, the exponential decay of the PDF, agreeing with literature results, is consistent for the three cases.

(a)
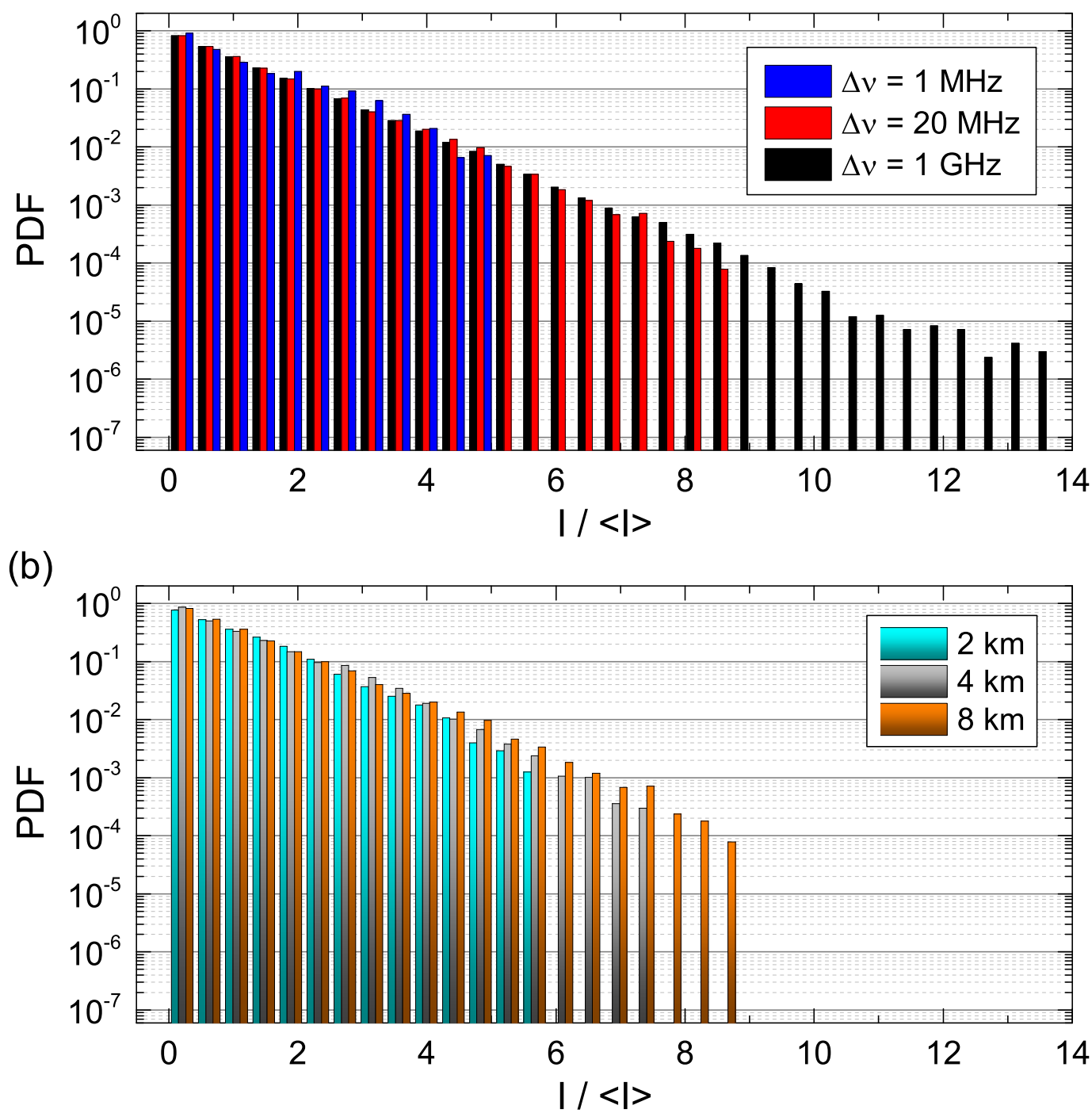

Figure 3.6: Probability density function of simulated Rayleigh backscattering intensity fluctuations in (a) an 8-km long fiber for different bandwidths and (b) for a fixed bandwidth of $20 \mathrm{MHz}$ and different fiber lengths (b).

A new set of PDFs was generated from simulations with a fixed bandwidth $(20 \mathrm{MHz})$ and for three different fiber lengths: 2,4 and $8 \mathrm{~km}$. The result 
is shown in Figure 3.6(b). It can be seen that longer fibers visited more extreme events than shorter ones. This is due to the stronger intensity fluctuation with optical frequency for longer fibers, as shown in Figure 3.3. Thus, to compensate the lack of extreme statistical events and obtain a more complete probability distribution one could either simulate Eq. 3-5 for wider bandwidths, or longer fibers, as both changes affect the probability distribution extension.

Last, we evaluated the impacts on the PDFs when the distribution of the random variable $\bar{n}_{i}$ is changed from uniform to Gaussian. In the same way as before, we have that $\bar{n}_{i}=1.44+\xi_{i} 10^{-7}$, but now $\xi_{i}$ is set as random variable with Gaussian distribution. Standard deviations of $\bar{n}_{i}$ were simulated from $\sigma=10^{-8}$ to $10^{-16}$. All simulations were performed for a 4 -km long fiber and a simulation bandwidth of $20 \mathrm{MHz}$. Results are presented in Figure 3.7. Standard deviations greater than $10^{-13}$ generated results similar to those obtained with a uniform distribution. This means that even extremely small residual stress can lead to fluctuation of the refractive index giving an exponential distribution of backscattered intensities.

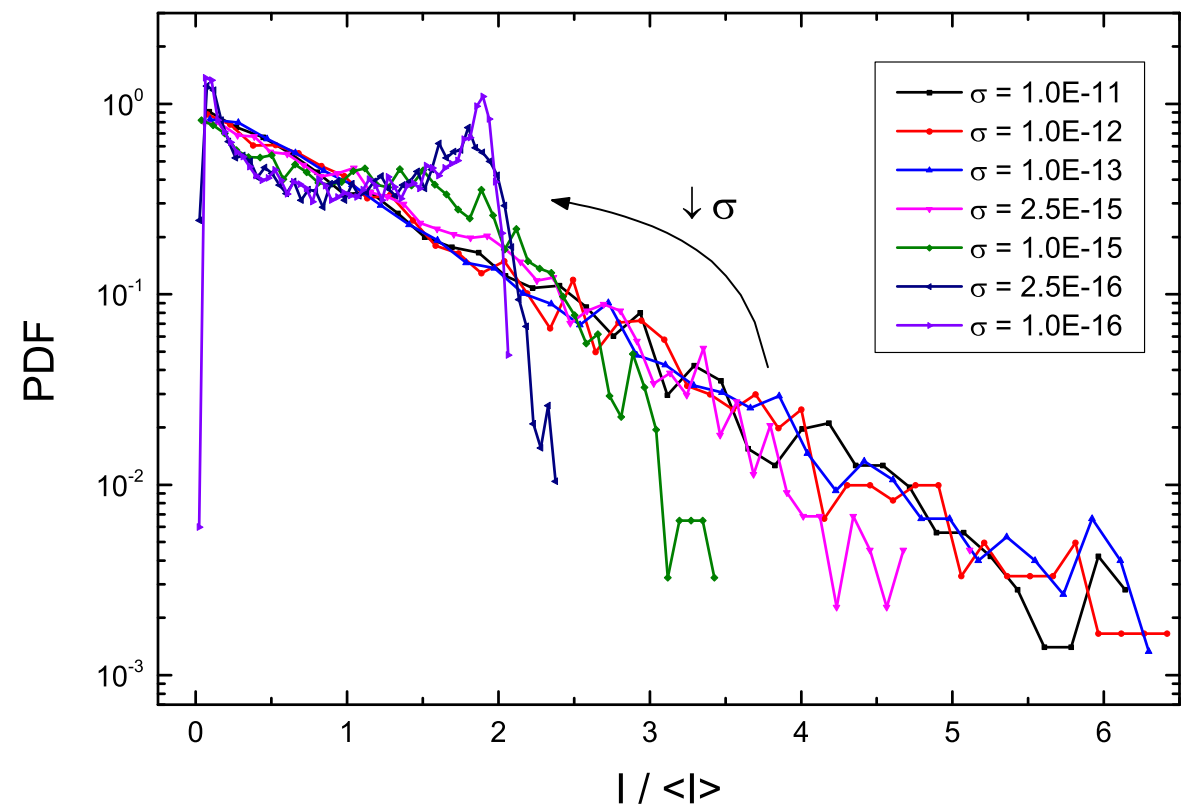

Figure 3.7: Simulated probability density functions of Rayleigh backscattering intensity fluctuations considering the refractive index with a random Gaussian distribution. When the standard deviation $\sigma$ of the index distribution reaches values below $10^{-13}$, it is observed a transition from an exponential PDF to a distribution of a sinusoidal variable.

For $\sigma$ 's smaller than $10^{-13}$, the PDFs' slope (in log scale) increases in absolute value and starts to diverge from that obtained experimentally. When $\sigma=10^{-16}$, the refractive index fluctuations are so small that the PDF tends to the distribution of a sinusoidal variable, complying with the result 
predicted from Eq. 3-3 using an average refractive index along the optical fiber. Two important conclusions are taken from these results: 1) a fading backscattering intensity is not constrained to a uniform distribution of the refractive index fluctuations as assumed in section 3.2.2; and 2) refractive index fluctuations much smaller than $10^{-7}$ can still lead to intensity fluctuations of the Rayleigh backscattered light. This second conclusion suggests that, even for a fiber manufacturing process with the loop control turned off, extremely small fluctuations of the fiber-pulling speed, or even tiny temperature fluctuations near the silica fictive temperature, would still result in a significant refractive index fluctuation, making the result in Figure 3.5(g) unfeasible. 


\section{3 \\ Ergodicity of Rayleigh backscattering}

Since the intensity of Rayleigh backscattered coherent light fluctuates in time, it can be classified as a random dynamical system. The statistical properties of random dynamical systems are often analyzed with the aid of ergodic theory. The fundamental objective of ergodic theory is to study the long-term statistical behavior of dynamical systems [50]. Although random processes necessarily involve the notion of change over time, the probability laws governing such changes may remain fixed as time passes [66]. For instance, the probability laws governing the rolling of a dice multiple times over a long time-period are constant provided that experimental conditions remain the same. From a qualitative viewpoint, ergodic theory can be seen as the divider between deterministic chaos and complete randomness [52].

To better define ergodic theory, let us consider an experiment that is performed periodically and has been going on forever and will continue forever - for example, a dice that is being throwing once a minute and has been thrown forever and will continue like so. This double infinite sequence of experiments can be represented as a major experiment $w$ such that

$$
w=\left(\ldots, w_{-1}, w_{0}, w_{1}, \ldots\right)
$$

where $w_{i}$ indicates the $i^{\text {th }}$ "smaller" experiment. The probability structure of the major experiment $w$ is described by a probability measure $P$ on the space $\Omega$. Let us consider another major experiment $w^{\prime}$ as the shifted replica of $w$ given by $\left(\ldots, w_{0}, w_{1}, w_{2}, \ldots\right)$. If the passage of time does not affect the probability laws governing the experiment, then $P(w)$ should be equal to $P\left(w^{\prime}\right)$. Thus, if $T$ is the transformation carrying $w$ to $w^{\prime}$, then $P(A)=P(T A)$ for sets $A$ of major experiments, hence the probability measure of the major experiment preserves transformations. The ergodic theory is that which studies systems with measure-preserving transformations [66].

The ergodic theory gave birth to the concept of ergodic process. We shall generally refer to Athanasios Papoulis and Unnikrishna Pillai [51] for a formal definition of ergodic processes. Let $\mathbf{x}(t)$ be a real stationary process with mean $\eta=E\{\mathbf{x}(t)\}$. The objective here is to estimate $\eta$ from an average over a finite time period $T$. The time-average over period $T$ is given by

$$
\eta_{T}=\frac{1}{T} \int_{-T / 2}^{T / 2} \mathbf{x}(t) d t
$$

Thus, $\eta_{T}$ is a random variable with mean

$$
E\left\{\eta_{T}\right\}=\frac{1}{T} \int_{-T / 2}^{T / 2} E\{\mathbf{x}(t)\} d t=\eta .
$$


If the variance of $\eta_{T}$ goes to zero as $T \rightarrow \infty$, then $\eta_{T} \rightarrow \eta$. That being the case, the time average from a single and long enough realization of $\mathbf{x}(t)$ would be approximately $\eta$, and $\mathbf{x}(t)$ is classified as an ergodic process. Thus, if the statistical properties of a stochastic process $\mathbf{x}(t)$ can be derived from a single and long enough random sample of $\mathbf{x}(t)$, then the process is said to be ergodic.

A simple example of ergodic behavior is presented in [52]. It was considered an experiment in the sample space $X=[0,1]$, with elements $\mathbf{x}(t) \in X$ given by $\mathbf{x}(t)=\sin ^{2}(t)$, where $t$ represents random sampling times. The probability measure $P$ is of an arc-sine distribution, with probability density function $p(\mathbf{x})=[\pi \sqrt{\mathbf{x}(1-\mathbf{x})}]^{-1}$. The logistic transformation $T$, mapping elements of $X$ to $X$, given by $T(\mathbf{x})=4 \mathbf{x}(1-\mathbf{x})$ is invariant with respect to $P$, thus configuring and ergodic process. The histogram of $\mathbf{x}(t)$ and its logistic mapping $T(\mathbf{x}(t))$ are shown in Figures 3.8(i) and (ii), respectively, where the former was obtained for a time interval two times longer than the latter. Note that, even for different but long enough time intervals, the statistics of the two experiments match, both presenting the same mean value.

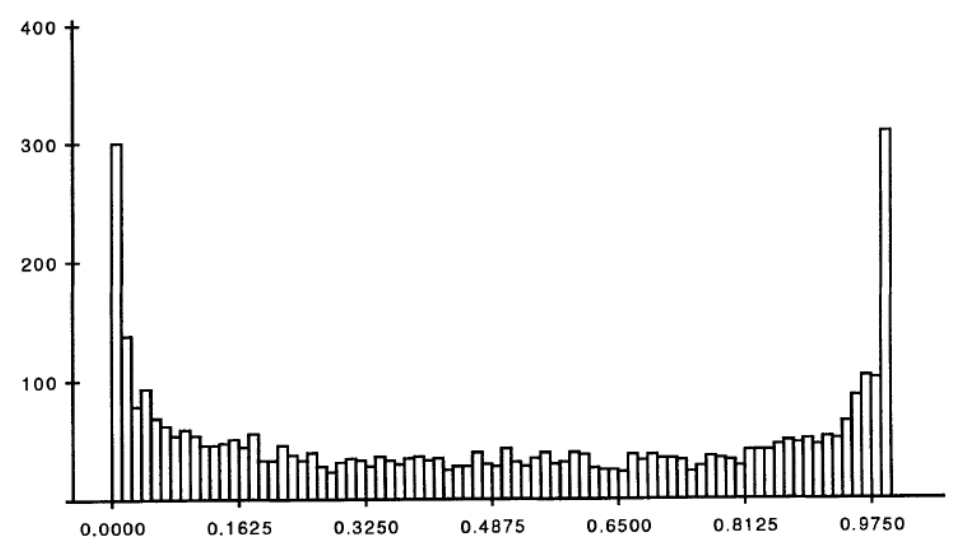

(i)

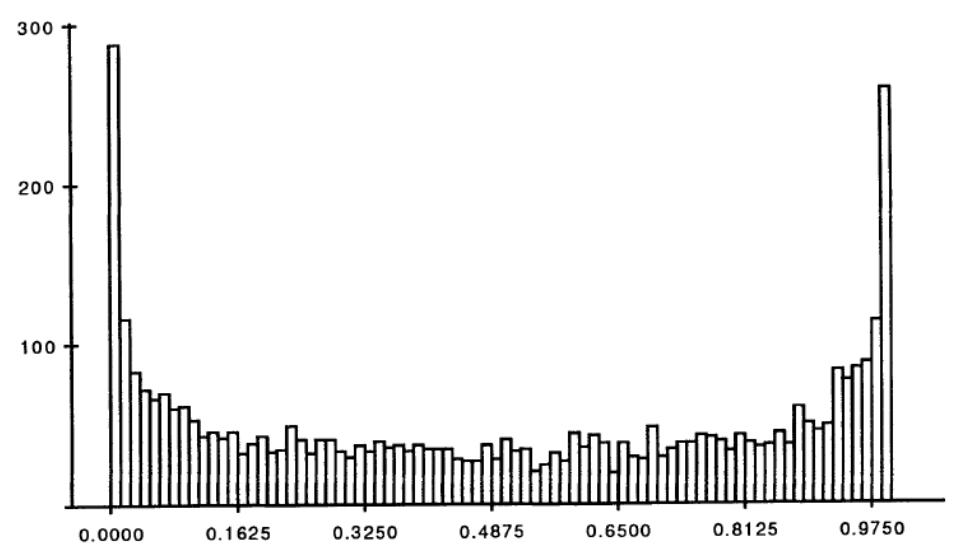

(ii)

Figure 3.8: Example of ergodic behavior. (a) Histogram of $x(t)$ for 4,000 samples, and (b) histogram of logistic mapping for 2,000 samples [52]. 
It should be clear that not all stochastic processes are ergodic. A straightforward example of a non-ergodic process is given as follows [51]. Suppose that $\mathbf{c}$ is a random variable with mean $\eta_{c}$ and $\mathbf{x}(t)$ is a stochastic process such that $\mathbf{x}(t)=\mathbf{c}$. Clearly, $\mathbf{x}(t)$ is a family of straight lines (constants), with mean $\eta=E\{\mathbf{x}(t)\}=E\{\mathbf{c}\}=\eta_{c}$. Over a time-interval $T$, the mean of the process is written as $\eta_{T}$. For a specific sample $\xi$ of $\mathbf{x}(t)$, if $\mathbf{c}(\xi)$ is different than $\eta_{c}$, then even for $T \rightarrow \infty, \eta_{T}$ will be different than $\eta_{c}$, thus the process is non-ergodic, as the mean does not converge for long samples.

An interesting property of ergodic processes is called ergodicity. Two experiments shall be considered to explain this property. In the first experiment, we observe the events of a random system evolving over time. In the second experiment, we observe the events of multiple replicas of the first experiment, but only at a given time point. Provided that the statistics of the first experiment match those of the second experiment, then it is said that the system exhibits ergodicity between time-space and the state space over which replicas were generated. For instance, in [30] Poole claims that polarization mode dispersion (PMD) in optical fibers exhibits ergodicity in the time-frequency sense. This means that the statistics of PMD measurements observed at a fixed time for a wide frequency bandwidth match those observed for a fixed frequency (or narrow frequency bandwidth) over a long time period. Another form of interpretation is that the observation of the phenomenon at multiple frequencies represents the observation of multiple replicas of the random system under analysis, and provided that a large number of replicas is considered, if the statistics taken in the frequency-domain match those obtained in time-domain, then the system exhibits ergodicity. This shows to be an extremely relevant property for PMD measurements, and it is in fact used in all PMD measurement techniques [54].

For low coherent sources, Staubli and Gysel [49] mathematically showed that the field amplitude of Rayleigh backscattered light is an ergodic process. It was shown that it exhibits ergodicity in the sense that the average of backscattered amplitudes derived from many similar fibers at a given time is identical to that of a single fiber averaged over a long time period. Although this is an interesting mathematical result, it is unpractical for real applications, not only because it considers only low coherence sources but also because a representative measurement of amplitude fluctuations for a single fiber takes a long time, and an average over multiple fibers is unreasonable.

In the following sections we developed experimental measurements of intensity fluctuations of Rayleigh backscattered coherent light to investigate if it is a process exhibiting ergodicity between time and frequency domains, in the same way as PMD does. 


\section{4}

\section{Experimental results and ergodicity validation}

The experimental setup shown in Figure 3.9 was used to measure intensity fluctuations of Rayleigh backscattering. A RIO ORION $1550 \mathrm{~nm}$ laser, with $3 \mathrm{~dB}$ linewidth of $3 \mathrm{kHz}$, was used as coherent light source. A beam splitter directs 90 percent of laser light to an 8-km long single mode fiber, which is much smaller than the laser's coherence length $(\sim 21 \mathrm{~km})$. An optical isolator (OI) at the end of the fiber prevents the overlap of unwanted reflections with Rayleigh backscattering. Light backscattered along the optical fiber interferes with 10 percent of laser light shifted in frequency by 80 $\mathrm{MHz}$ at an acousto-optic frequency shifter (AOFS). A $125 \mathrm{MHz}$-bandwidth photodiode (PD) performs opto-electrical conversion. A $20 \mathrm{MHz}$ band pass filter (BPF) selects the intensity fluctuations superimposed onto the $80 \mathrm{MHz}$ tone, filtering out base-band noise. An $8 \mathrm{GHz}$ bandwidth electrical power detector ensures that only intensity/power fluctuations are measured, and it transfers intensity variations to the baseband. Finally, the power detector's output signal is measured in a 1-GHz bandwidth oscilloscope (OSC). Thus, the setup bandwidth was limited to $20 \mathrm{MHz}$ due to the band-pass filter.

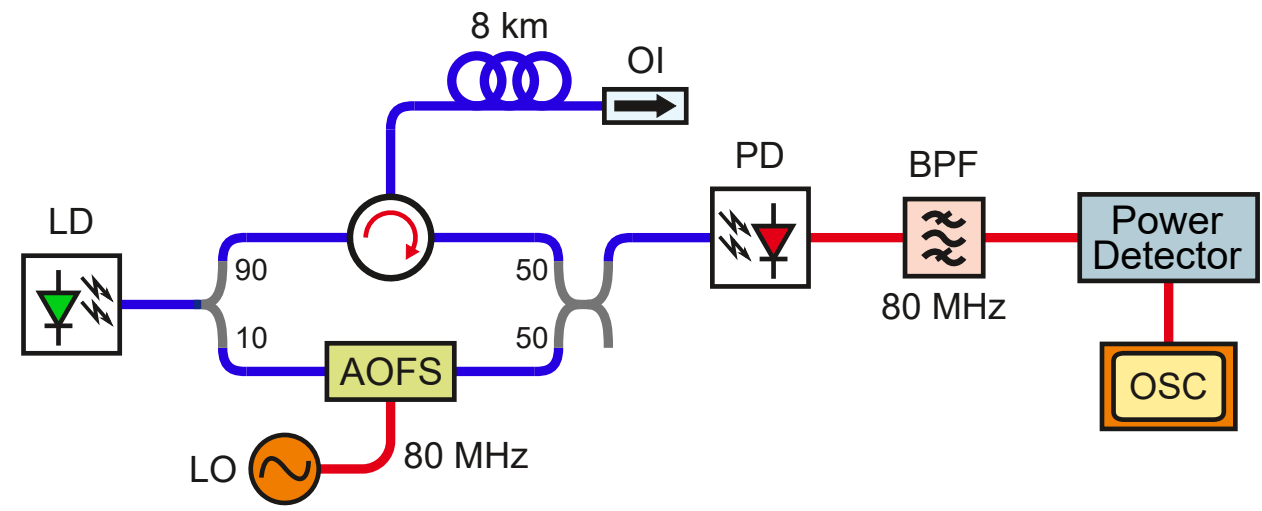

Figure 3.9: Experimental setup for measurement of intensity fluctuations of Rayleigh backscattering. AOFS, acousto-optic frequency shifter; BPF, bandpass filter; LO, local oscillator; OI, optical isolator; OSC, oscilloscope; PD, photodiode.

The usage of the experimental setup shown in Figure 3.9 was essential for measuring pure intensity fluctuations of Rayleigh backscattering, where the AOFS plays an important role. If the measurement was performed at baseband, without interfering with light from the bottom arm of the beam splitter, then phase variations of the source could jeopardize the measurement. For instance, the phase jitter or wandering of the light source would make contributions from different parts of the fiber to interfere at PD, whereas with heterodyne 
beat this source phase contribution is canceled. Moreover, this set up brings benefices as it provides the higher sensitivity of interferometric detection.

If one were to measure the intensity of light backscattered by a frozen fiber, a constant intensity value would be obtained. In a real scenario, environmental changes along the optical fiber alter its refractive index locally, what ends up modifying continuously the backscattered optical field. In [67], authors show that the phase of an $8 \mathrm{~km}$ long fiber fluctuates due to environmental factors in milliseconds time scale, whereas slower changes are observed in shorter fibers. If environmental changes are indeed the cause of fluctuations in Rayleigh backscattering intensity, then, provided that a coherent source is used, the backscattering intensity should oscillate in the same way as the optical phase, i.e., it should fluctuate more for longer fibers. This assumption was experimentally tested by measuring the frequency content of the Rayleigh backscattered signal for fibers with lengths of 1, 2, 4 and $8 \mathrm{~km}$ by calculating the FFT of the measured backscattered intensity. The results are shown in Figure 3.10, with power normalized by the maximum value. It is noticeable that longer fibers present fluctuation frequencies much higher than shorter ones. Indeed, there is a limit on the fluctuation frequencies as the fiber length is increased because the contributions to intensity fluctuations from fiber sections farther from the input end are exponentially attenuated.

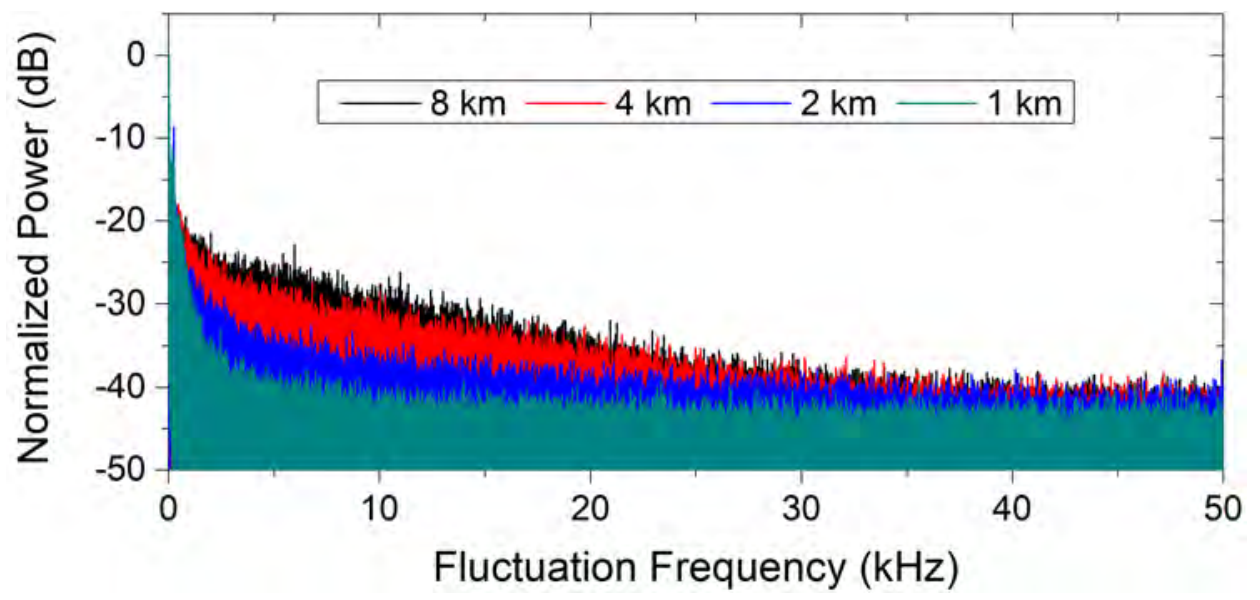

Figure 3.10: Spectral measurement of Rayleigh backscattering intensity fluctuations from 1, 2, 4 and $8 \mathrm{~km}$ fibers.

Spectral measurement results shown in Figure 3.10 indicate that an oscilloscope with frequency bandwidth greater than $\sim 40 \mathrm{kHz}$ suffices for the measurement. The oscilloscope acquisition rate was set to $100 \mathrm{kHz}$ to allow measurements over $100 \mathrm{~s}$, thus reducing the set-up bandwidth to $50 \mathrm{kHz}$ without jeopardizing the measurement. In the same way that intensity fluctuations in the frequency domain required wider bandwidths for seizing extreme intensity 
values, experimental signal acquisition for longer times gives extended statistical information. The probability density function of the 100-s long measurement of intensity fluctuations from the $8 \mathrm{~km}$ fiber is provided in Figure 3.11, where intensities are normalized by the mean value. As expected for a Rayleigh process, an exponential distribution was found. The simulation result obtained in section 3.2.3 for a bandwidth of $1 \mathrm{GHz}$ is plotted in blue for comparison. Interestingly, experimental and simulated PDFs show good similarity, though the former was obtained in the time domain, and the latter in the frequency domain. A valid comparison/discussion between time and frequency-domain results will soon be provided, but first, to better represent the experimental measurement, the theoretical model should be modified to include the laser linewidth.

The model described in section 3.2.2 calculates the theoretical backscattered intensity for single frequencies, i.e. as if it was used a monochromatic light source. The model must be corrected to account for real sources, with finite linewidths. Considering that source phase fluctuations were eliminated from the measurements, the light source with linewidth $\Delta \nu$ can be considered as an incoherent sum of light waves with weighted intensities within $\Delta \nu$, so the resultant backscattered intensity for a given frequency $\nu$ corresponds approximately to the averaging of intensities across the interval $[\nu-\Delta \nu / 2, \nu+\Delta \nu / 2]$. For instance, if a light source with $5 \mathrm{MHz}$-linewidth was used to simulate results in Figure 3.3, then the high frequency fluctuations of the $1 \mathrm{~km}$-long fiber would be averaged out across $5 \mathrm{MHz}$ for every optical frequency. Thus, instead of using a single $\nu$ (or $k$ ) in Eq. 3-5 to calculate the intensity for a given frequency, the intensity should be calculated considering the lineshape function of the coherent source centered at each frequency $\nu$. This can be performed in two steps: i) calculating the intensity for a monochromatic light swept across the simulation bandwidth (direct application of Eq. 3-5), followed by ii) a moving average algorithm weighted by the light source's lineshape. Modified backscattered intensity $I_{b}$ is written as:

$$
I_{b}(z=0, \nu)=\frac{1}{M} \sum_{j=1}^{M}\left|E_{b}\left(z=0, \nu_{j}\right)\right|^{2} \cdot w_{j},
$$

where $M$ is the number of data points to be considered in a window of the moving average, and $w_{j}$ the weight of lineshape function at optical frequency $\nu_{j}$. For improved accuracy, $M$ should be large enough to include at least a 10-dB bandwidth of the lineshape function.

To incorporate in the simulation the same light source used in the experiment, with a Lorentzian lineshape and $3 \mathrm{kHz}-$ linewidth, Eq. 3-9 was used. It was considered the same fiber length of the experiment, $L=8 \mathrm{~km}$, a 
frequency bandwidth of $1 \mathrm{GHz}, f_{i}=250 \mathrm{~Hz}$, and $M=49$, such that the interval $\nu_{M}-\nu_{1}$ is four times the laser linewidth, equivalent to $\sim 12 \mathrm{~dB}$-bandwidth. The PDF of simulated intensity fluctuations corrected by laser linewidth is shown in Figure 3.11.

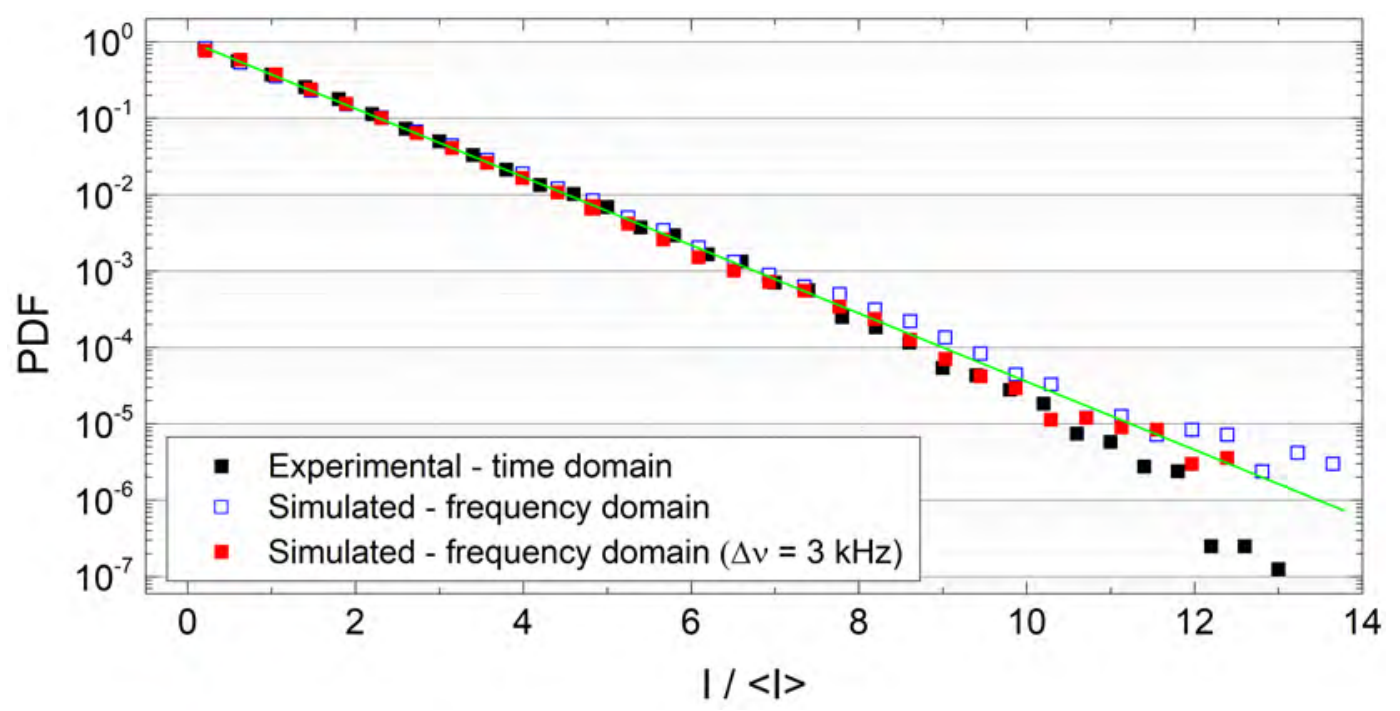

Figure 3.11: Probability density functions of Rayleigh backscattering intensity fluctuations evaluated in time (experimental), and frequency domains (simulation). Intensities are normalized with respect to the mean value.

The comparison of statistical results, regarding the same dynamical phenomenon, but evaluated at the different measurement spaces of time and frequency, is valid only if the phenomenon is an ergodic process exhibiting ergodicity in the time-frequency sense. That being the case, if samples taken from time and frequency domains are long enough to be representative of the entire phenomenon, and provided that their averages are the same, then their statistics should match.

As shown in Figure 3.11, the coincidence between the experimental measurement in time domain and corrected simulation result in frequency domain is remarkable, strongly supporting the statement that Rayleigh backscattering in optical fibers is indeed an ergodic process with ergodicity between time $\&$ frequency domains. The agreement between the two PDFs extends to five decades. For extreme intensity values, approximately ten times above the mean intensity, the PDFs starts diverging. This effect is probably due to the lack of extreme events in the simulation, in the experiment, or in both. Expanding the measurement/simulation spaces by measuring intensity fluctuations for times longer than $100 \mathrm{~s}$ and using simulation bandwidths wider than $1 \mathrm{GHz}$ would result in more complete statistics. Ideally, an infinitely long measurement-time and an infinitely wide simulation-bandwidth would bring both PDFs closer 
to the theoretical exponential distribution, which is shown as a green line in Figure 3.11.

To verify if the behaviors observed in the simulated PDFs shown in Figure 3.6 comply with experimental measurements, i.e., wider bandwidths and longer fibers resulting in more complete statistics, two experiments were performed. First, supported by ergodicity, simulations with wider bandwidths should be equivalent to measurements in longer times. Intensity fluctuations were then acquired for three different times intervals, $100 \mathrm{~ms}, 2 \mathrm{~s}$ and $100 \mathrm{~s}$, and the correspondent PDFs are shown in Figure 3.12(a). Results are clearly compatible with those from Figure 3.6(a). It is relevant to highlight that, although the same proportion of time intervals and frequency bandwidths were used, it does not mean that there is a perfect equivalence between them, for instance, $1 \mathrm{GHz}$ and $100 \mathrm{~s}$ are not necessarily equivalent in terms of statistical content. Were the environmental fluctuations slower than those found in the laboratory experiments, longer times would be needed to achieve the same statistics. The conclusion here is that a reduction/increase in simulation bandwidth is equivalent to a reduction/increase in measurement time. Second, intensity fluctuations were measured using a constant time interval (100 s) and for fibers with lengths of 2, 4 and $8 \mathrm{~km}$, the same lengths used in simulations presented in Figure 3.6(b). The PDFs from these experimental measurements are shown in Figure 3.12(b). As expected from the simulation results, longer fibers indeed visited more extreme events, providing more accurate statistics. The comparison of absolute intensity values between simulation results in Figure 3.6(b) and experimental results in Figure 3.12(b) indicates that the simulation bandwidth of $20 \mathrm{MHz}$ was not enough to reach the same statistical extension obtained with the experimental measurement for $100 \mathrm{~s}$. It is worth to mention that the measurements in Figure 3.12(b) are not limited by the bandwidth of the measurement set-up. In fact, a peak intensity as high as 13 times above the mean value with probability $10^{-7}$ in $100 \mathrm{~s}$ would last $\sim 10 \mu$ s which is still within the Nyquist limit of the data acquisition rate. Going further on with low probability events would demand stacking 100-s long data streams or increasing the memory capacity of the oscilloscope.

It is interesting to note that the exponential distribution displayed in Figures 3.6, 3.11 and 3.12, which agrees with many experimental reports in the literature $[25-27,48]$, is due to the very nature of the scattering phenomenon. In fact, the interference of the backscattered optical fields along the single mode fiber is analogous to the interference of radio waves coming from multiple paths scattered between the transmitter and receptor in a radio link. From communication theory, the radio-signal fluctuations can be described by the 

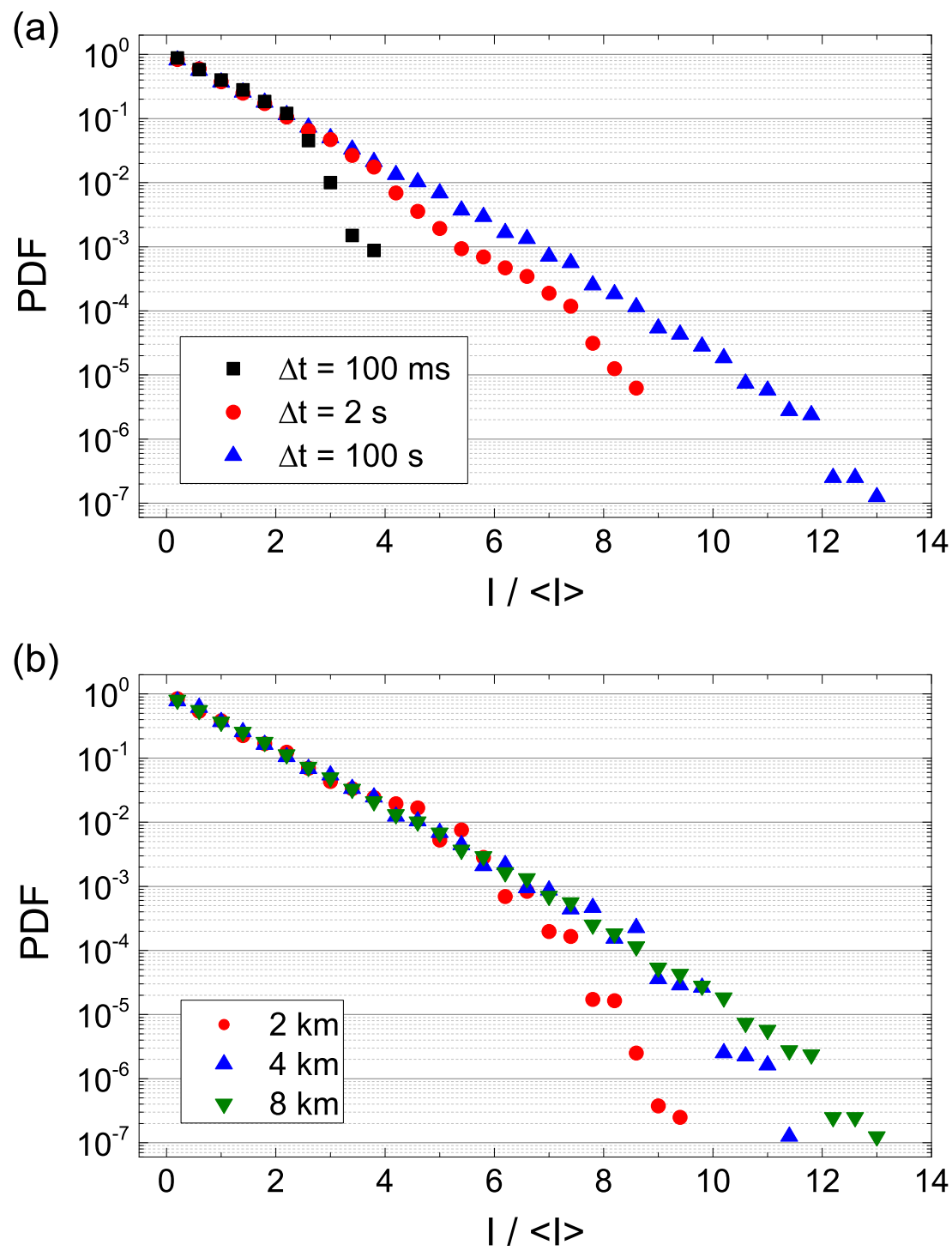

Figure 3.12: Experimentally obtained probability density function of Rayleigh backscattering intensity fluctuations for different acquisition intervals (a) and for different fiber lengths (b).

Rayleigh distribution [68], which is a special case of the Weibull distribution. It is long known that Rayleigh distribution describes well the field superposition of a number of waves [69], and to analyze the intensity/power fluctuations one should take the absolute square value of the superposed fields, resulting in a Rayleigh power distribution or exponential distribution [70]. Thus, the exponential distributions obtained in this work agree well with the statistical theory of superposed fields with random phases.

A mathematical proof that Rayleigh backscattering in single mode optical fibers exhibits ergodicity between time-frequency domains is rather complex and out of the scope of this work, however, an explanation for this affirmative can be supported by the same argument used in [30] to justify the ergodicity of polarization mode dispersion. The field of backscattered Rayleigh light coming 
from a certain point of an optical fiber depends on the frequency of light at that point. Hence, a change in the frequency of the light source alters the backscattered Rayleigh contributions throughout the optical fiber. Therefore, a frequency perturbation is everywhere representative of the ensemble of Rayleigh contributions. Thus, the statistical behavior of Rayleigh intensity fluctuations for a given frequency in time, is equivalent to that for a given time in all frequencies. However, as shown in Figure 3.11, a bandwidth of $1 \mathrm{GHz}$ is already sufficient for a good representation of the phenomenon in the frequency domain.

The validation of ergodicity for Rayleigh backscattering in optical fibers is extremely relevant for many research areas. For instance, it can help researchers in $\Phi$-OTDR to rapidly obtain the statistics for the best spatial resolution through simulations in the frequency domain. For fiber-optic distributed temperature sensors based on Rayleigh scattering, the ergodicity property can be used to determine statistically the best achievable temperature resolution. 


\section{4}

\section{Random DFB fiber lasers}

\section{1}

\section{Introduction to random lasers}

As briefly discussed in chapter 1, random lasers have the same building blocks of conventional lasers: a gain medium and a feedback mechanism. Despite this fundamental similarity, the build up of laser action is quite different. The optical cavity of conventional lasers discussed in section 2.1.2 determines the allowed longitudinal modes in the resonator, which have narrow spectral width, strong directionality, high degree of polarization and well defined spatial distribution. In this case, it is said that the feedback is coherent because the optical field interferes within the resonator, defining a free spectral range given by $c /(2 d)$ (see Eq. 2-9). Random lasers are characterized by not having a well-defined cavity, which is often referred to as open-cavity [71-73]. However, a feedback mechanism is still required to permit laser action. The feedback is promoted by multiple scatterings, which randomize the optical path undertaken by light in the gain medium. In a gain medium with strong scattering, light will undergo multiple scatterings before escaping the gain volume, thus light is trapped for a time longer than that in a free optical path, and the dwell time under optical gain is increased. It has been shown that the integral gain is proportional to the volume of the scattering medium, while the integral losses are proportional to the surface area of the scattering medium $[74,75]$. Therefore, by increasing the pumping of the gain medium, eventually the gain will overcome the losses, when a lasing threshold is reached. Indeed, in the first experimental proof of random lasing, Lawandy and co-workers used a suspension of $\mathrm{TiO}_{2}$ particles in a rhodamine 640 perchlorate laser dye [7] to show that the lasing threshold is defined by the density of scatterers, such that mediums with more scatterers provide stronger feedback resulting in a lower lasing threshold.

Figure 4.1 [10] shows schematically the lasing action in random lasers. Scatterers randomly positioned in the gain medium are represented by gray spheres, and red arrows indicate random optical paths. A typical spatial distribution of intensities is shown in a jet color-map on the left [76]. Different 


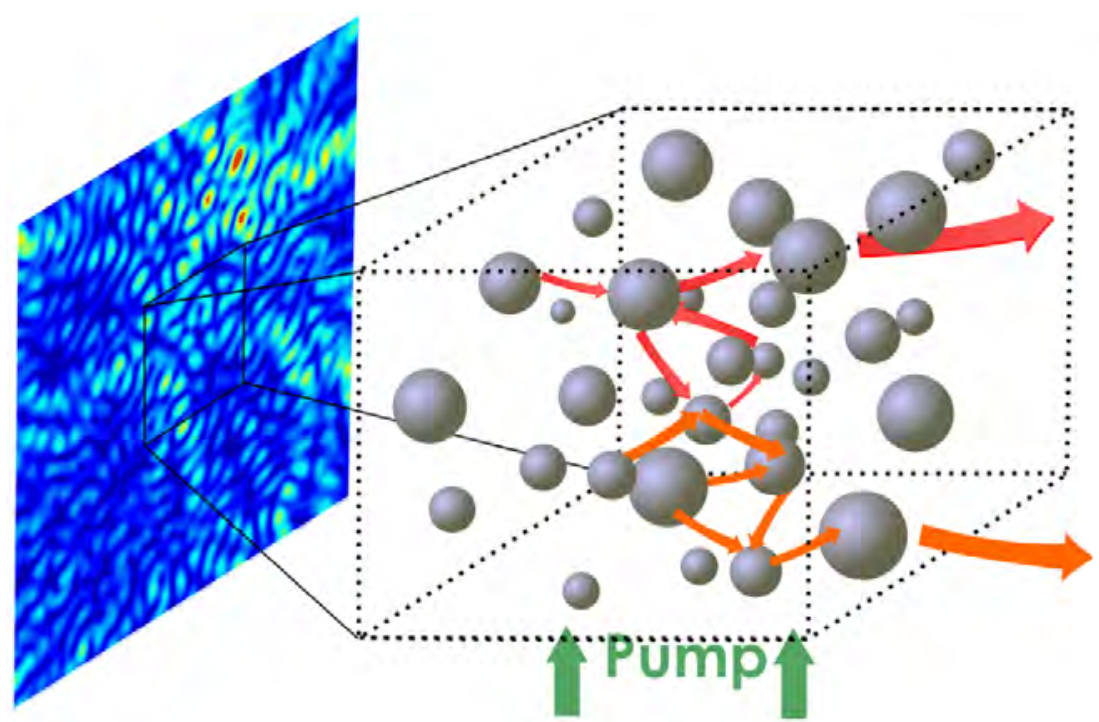

Figure 4.1: Schematic of random lasing [10]. Multiple scatterers traps the light in a confined gain volume, increasing the effective amplification path undergone by light. Open random paths determine an incoherent feedback, whereas closed paths allow coherent feedback. The panel on the left corresponds to a simulation of power spatial distribution [76].

than conventional lasers, which exhibit a well-behaved spatial distribution (normally Gaussian), the spatial distribution of random lasers is characterized by a random power distribution, which is a fingerprint of random lasers. Another fingerprint of random lasers is the chaotic emission spectrum. As the direction of light is changed randomly at every scattering center, light does not return to its original position, precluding the constructive interference of optical fields. Thus, random lasers configure a case of incoherent feedback, which is intensity-based. Hence, the emission spectrum is governed by the gain medium characteristics, assuming a linewidth $\Delta \nu$. However, $\mathrm{H}$. Cao has shown $[77,78]$ that a strong scattering can lead to resonant feedback, when light returns to an origin position interfering constructively (field-based). A stronger scattering increases the probability of light returning to an original position, thus closed loops, or micro-cavities, can arise more likely. Multiple closed loops can be formed across the gain medium, such that coherent feedback is obtained for multiple frequencies. These can be thought of as micro-lasers, which promote coherent emission at random frequencies [33]. The formation of closed loops results in narrow lasing spikes on the top of the gain profile, and the frequencies of spikes vary for each single-shot measured spectrum. Figure 4.2 shows a sequence of typical emission spectra of a random laser, which in this particular case was built from a powder of SK11 (Schott) glass inserted in a solution of rhodamine-6G and optically pumped [79]. Even though the coherent emission is build on top of the gain profile, since it extends for 
several nanometers narrow spikes can arise at multiple frequencies, resulting in a emission spectra highly uncorrelated.

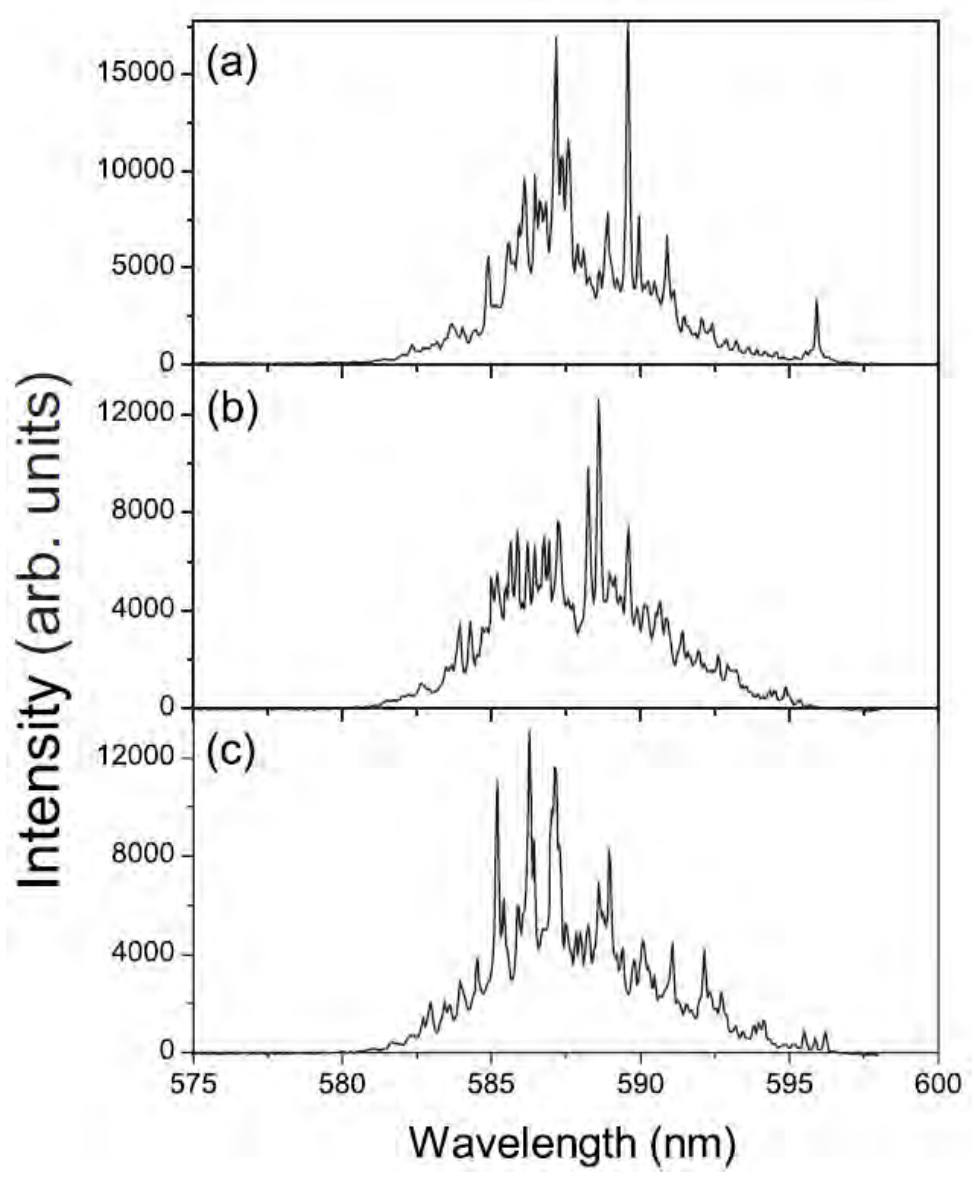

Figure 4.2: Three successive measured spectra of a random laser composed of a porous glass doped with laser dye. The chaotic behavior of emission spectra is observed, where no correlation is found between successive spectral measurements [79].

The possibility of having coherent feedback in multiple frequencies have led authors to classify random lasers as those with a "modeless" spectrum [14, 71]. Although the intention is clearly to state that no frequency prevails over the gain profile, it might be a misleading definition as one might think that not a single mode is satisfying the lasing conditions. For high enough pumpings, laser action can build up for nearly all frequencies under the gain profile at the same time, giving a continuous multimode laser [12]. Even so, it is relevant to highlight that this is a topic not fully understood and under continuous discussion. In fact, we quote here a statement from [10] about the modes in random lasers: "The nature of lasing modes in random scattering active media is a fascinating research problem that is still under extensive studies and discussions."

Random lasers can be developed in many media with disordered struc- 
ture. When solid state lasers are ground into powders, for instance, $\mathrm{Nd}^{+3}$ :YAG, $\mathrm{Nd}^{+3}$ :glass, $\mathrm{Ti}^{+3}$ :sapphire, and GaAs, then random laser action can be observed [33]. There are numerous active media that have been used to generate random lasers, including powders, granular materials, porous glasses, inorganic dielectrics, polymers, liquids, dye solutions, dye-doped liquid crystals, disordered semiconductor nanostructures and biological tissues [10]. A particularly important medium where random laser action can build up is in the amorphous structure of optical fibers $-\mathrm{SiO}_{2}$ glasses. As seen in chapter 3, Rayleigh backscattering is present in optical fibers due to tiny density fluctuations along the fiber. It will be shown in the following section that, however small, this backscattering can lead to random laser action.

\section{2}

\section{Review of random DFB fiber lasers}

Random DFB fiber lasers are a subset of random lasers, and for a detailed review of the larger set of random lasers we direct the reader to excellent reviews in $[8,78,80-83]$. This section reviews the main concepts of random DFB fiber lasers that are relevant for this thesis, and it is organized as follows. Section 4.2.1 presents the theoretical background of random DFB fiber lasers. Next, characteristics of spectral and temporal dynamics are given in section 4.2.2. Last, random DFB fiber lasers with other types of gain are presented in section 4.2.3.

\subsection{1}

\section{Theoretical background}

As discussed in section 4.1, traditional random lasers are characterized by a chaotic emission, which is observed in temporal, spectral, and spatial domains. One way to reduce the randomness in power spatial distribution is to confine the feedback to lower dimensions. This can be obtained by using optical fibers as the medium for random lasing, such that the multiple scatterings showed in Figure 4.1 in a three dimensional volume are confined to nearly one dimension. The confinement is caused by the refractive index difference between core and cladding structures, which traps light in the direction transverse to propagation. The benefits of using optical fibers as the medium for random laser action goes far beyond the reduction in complexity of spatial emission, which, in this case, assume a nearly Gaussian beam.

Although there are many works reporting random fiber lasers with hollow core optical fibers, which are normally filled with solutions encompassing multiple scattering particles to allow random laser action $[9,84,85]$, here we 
will focus on a particular type of random fiber laser, the so called random DFB fiber laser. Random DFB fiber lasers make use of Rayleigh scattering for coherent feedback, while different gain mechanisms have been employed - covered in details in section 4.2.3. For now, we will proceed the discussion making use of the first reported gain mechanism for random DFB fiber lasers, a distributed Raman gain [1]. As discussed in chapter 3, Raman scattering is a form of light-matter interaction. Stimulated Raman scattering (SRS) has been used in numerous optical fibers systems to provide optical gain, which is obtained by converting a pump radiation in amplified stimulated emission at the shifted Stokes wavelength, which has maximum value $\sim 13 \mathrm{THz}$ apart from the pumping wavelength [2]. Figure 4.3 shows a schematic of both feedback and gain mechanisms of a random DFB fiber laser, i.e., Rayleigh scattering and Raman gain, respectively. Pumping is introduced in both directions of an optical fiber, providing bi-directional Raman gain. In the same way, Rayleigh backscattering promotes coherent feedback in both directions. According to [1], there is a trade-off imposed by fiber length. An extremely short fiber would not be able to promote Raman gain, neither a significant feedback by Rayleigh scattering. On the other hand, an ultra-long fiber would severely attenuate the output signal. Thus, the fiber length should be carefully designed for optimal operation. A detailed discussion of this topic is provided in [71].

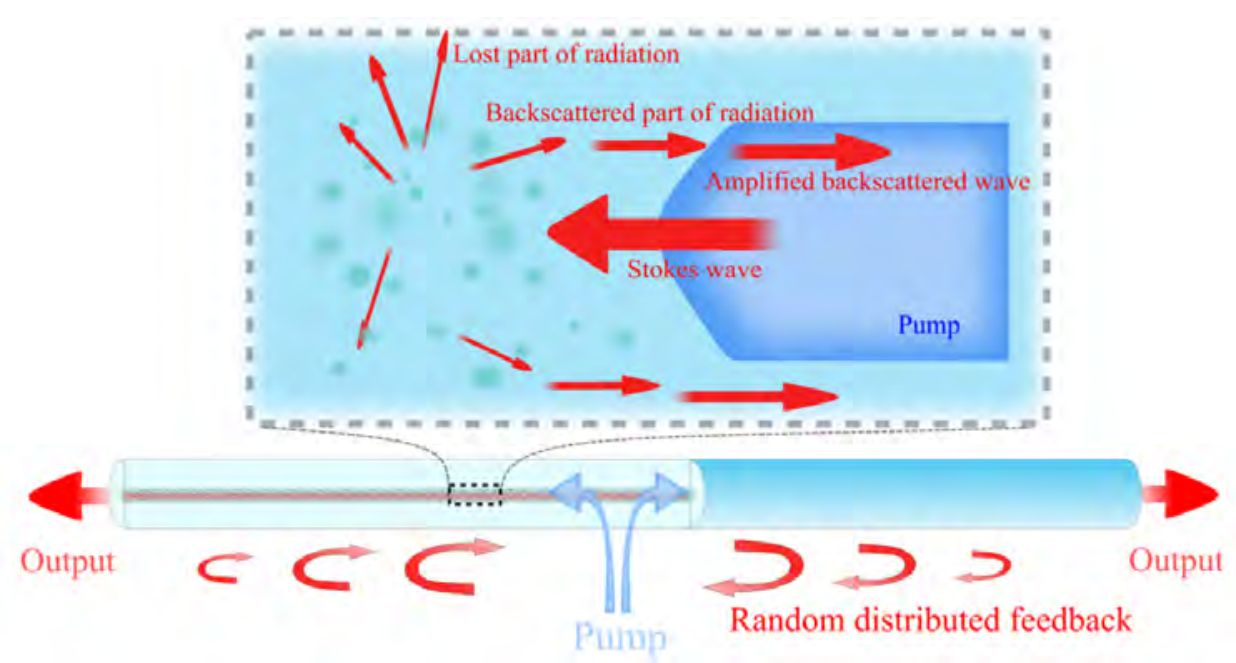

Figure 4.3: Random distributed feedback fiber laser. An optical fiber provides distributed Raman gain when pumped by two lasers. Distributed Rayleigh backscattering promotes coherent feedback along the optical fiber, defining an open optical cavity. With light trapped in the transverse plane of propagation direction, the distributed feedback and gain mechanisms can generate random laser action, which is thus observed in a one-dimension structure [10].

The usage of Rayleigh scattering as the feedback mechanism for random DFB fiber lasers is something quite unique compared to traditional random 
lasers, where strong scattering is used to promote laser action. Standard optical fibers are designed to have minimum losses at the telecommunication window of $1.55 \mu \mathrm{m}$, which are mainly defined by Rayleigh scattering, imparting an attenuation of $\sim 0.2 \mathrm{~dB} / \mathrm{km}$. As discussed in chapter 3 , it is possible to calculate the backscattered optical intensity (or optical field, see Eq. 3-5), and thus the reflectivity of optical fibers given by Rayleigh backscattering. Assuming an incoherent light source, the reflectivity of optical fibers can be calculated approximately by using the solid angle defined by its numerical aperture. Depending on the the fiber's composition and geometrical dimensions, fibers with lower or higher reflectivities can be manufactured. Still, it should be clear that longer fibers would promote stronger feedbacks, thus, the fiber's reflectivity is a quantity dependent of fiber length. According to [54], the power reflectivity $R$ for a fiber with length $L$ can be calculated by:

$$
R(L)=\frac{S \alpha_{s}}{2 \alpha} \cdot[1-\exp (-2 \alpha L)],
$$

where $S$ is the power fraction of light that is scattered in all directions and captured by the fiber core in the back direction, $\alpha_{s}$ is the attenuation coefficient related to scattering losses, and $\alpha$ is the total attenuation coefficient, given by $\alpha=\alpha_{s}+\alpha_{a}$, where $\alpha_{a}$ is the absorption coefficient. For standard single mode fibers at $\lambda=1550 \mathrm{~nm}$, the calculation of Eq. $4-1$ results in a reflectivity of approximately $-52 \mathrm{~dB}$ for a fiber with $100 \mathrm{~m}$ [54]. Interestingly, this weak scattering can result in random lasing provided that sufficient gain is employed.

This special random laser with weak scattering showed to be efficient, narrowband, with relatively low threshold $(\sim 1 \mathrm{~W})$ and stable CW lasing at $1.55 \mu \mathrm{m}$ for pumping powers higher than the threshold $(\sim 1.2 \mathrm{~W})[1]$. Near the generation threshold, narrow spikes are observed in the output spectrum, which have been associated with a cooperation between Rayleigh scattering and stimulated Brillouin scattering (SBS) [23]. Significantly above the threshold, a stable CW operation was obtained. Authors qualitatively argued that due to the increase in pumping power, nonlinear effects leads to spectral broadening, hence, lowering power spectral density and suppressing the SBS process, resulting in a stable operation. We propose to discuss the mode dynamics of this emission in more details, which we expect to show that it actually encompasses a more complex distribution of modes compared to conventional CW lasers. Since the feedback promoted by Rayleigh scattering is distributed along the optical fiber, in theory, all frequencies defined by the position of each fiber section would define a different mode. Thus, although the reflectivity of a short fiber section is much smaller than that of a highly reflective mirror, or that of an FBG, Rayleigh backscattering can be thought of as a sum of multiple 
monochromatic DFB lasers, resulting in a continuous multi-frequency output.

\subsection{2}

\section{Spectral and temporal dynamics}

No different than traditional random lasers, random DFB fiber lasers also exhibit a chaotic behavior in both temporal and spectral domains when pumped close to the laser threshold. However, it has been shown that under high pumping the emitted spectrum is smoothed, not showing narrow spikes, and the chaotic pulses in time domain are no longer observed, being replaced by a continuous wave (CW) operation [1]. This is a huge difference compared to traditional random lasers, since for applications like $\Phi$-OTDR, optical fiber sensing and others, it is key to have a stable optical source.

The spectral difference between unstable and stable emission is shown in Figure 4.4 [1]. The experimental setup considered was the same shown in Figure 4.3, with Raman scattering providing gain and Rayleigh scattering from an 83-km long fiber promoting coherent feedback. Output spectra were measured for pumpings close to the threshold $\left(\sim 1.2 I_{t h}\right)$, and far above (up to $\sim 1.7 I_{t h}$ ), which are shown in Figs. 4.4(a) and (b), respectively. Near the threshold, narrow spikes are observed on the top of Raman gain profile, which is characterized by two maximums at $\sim 1557$ and $1567 \mathrm{~nm}$. For pumpings significantly above the threshold, the spectrum shows a smooth profile, governed by the gain spectral width.

The transition between the chaotic to stable operation in time domain was approached in more details by Zhang and co-workers [86]. It was used an Yb-doped fiber laser (YDFL) for pumping at $1120 \mathrm{~nm}$, generating Raman gain near $1180 \mathrm{~nm}$. In the same way as in [1], feedback was provided by Rayleigh backscattering, but in Zhang's work, a much shorter optical fiber was used, with length of $300 \mathrm{~m}$. Thus, much higher pumping was required to generate random laser action. Interestingly, the reported spectra did not show narrow spikes near the threshold, indicating the absence of SBS. Indeed, calculations showed that the SBS threshold for the implemented set-up is about $90 \mathrm{~mW}$, a far higher level than that obtained in the experiments, thus SBS is not present in the cavity. However, a noisy emission was observed in the time domain near the threshold, and nearly stable operation was only found under high pumpings. Figure 4.5(a)-(d) shows oscilloscope shots with duration of $2 \mathrm{~s}$ for four different pumping powers, 49.2, 59.2, 72.1 and 85.6 W, respectively. Note that, the mean amplitude level when pumping at $49.2 \mathrm{~W}$ is about 0.5 (a.u.). By increasing the pumping, the mean level also increases, probably because the short random pulses observed in Figure 4.5(a), for instance at $1.85 \mathrm{~s}$, arise 

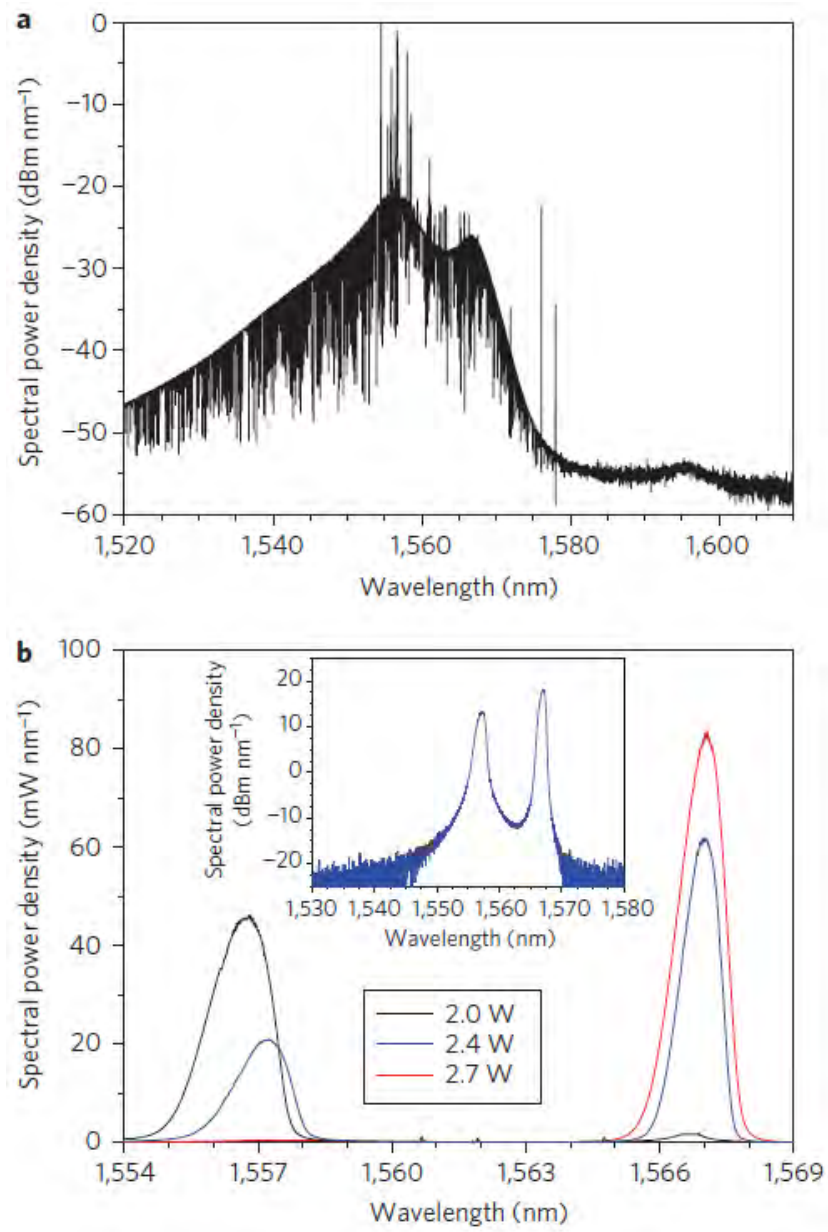

Figure 4.4: Optical spectra of random DFB fiber laser [1]. Pumping near (a) and significantly above the generation threshold (b).

more frequently. The overlap of such pulses would considerably increase the mean amplitude, which, at very high pumping shown in Figure 4.5(d), reaches 8 (a.u.) in a nearly CW operation.

Thus, although stable CW operation has been reported for random DFB fiber lasers under high pumping, we expect to provide a better understanding of the regime behind seemingly stable CW operation. By carefully taken experiments in time domain and frequency domains, we expect to investigate the interference between adjacent modes beating within the measurement bandwidth. Also, the theoretical model described in section 3.2.2 would be helpful to comprehend the real nature of the stable CW operation.

\section{2 .3}

\section{Other gain mechanisms}

After the first random DFB fiber laser was proposed with distributed Raman gain, different gain mechanisms were employed to verify random laser action. Indeed, multiple works report the generation of random lasers with 

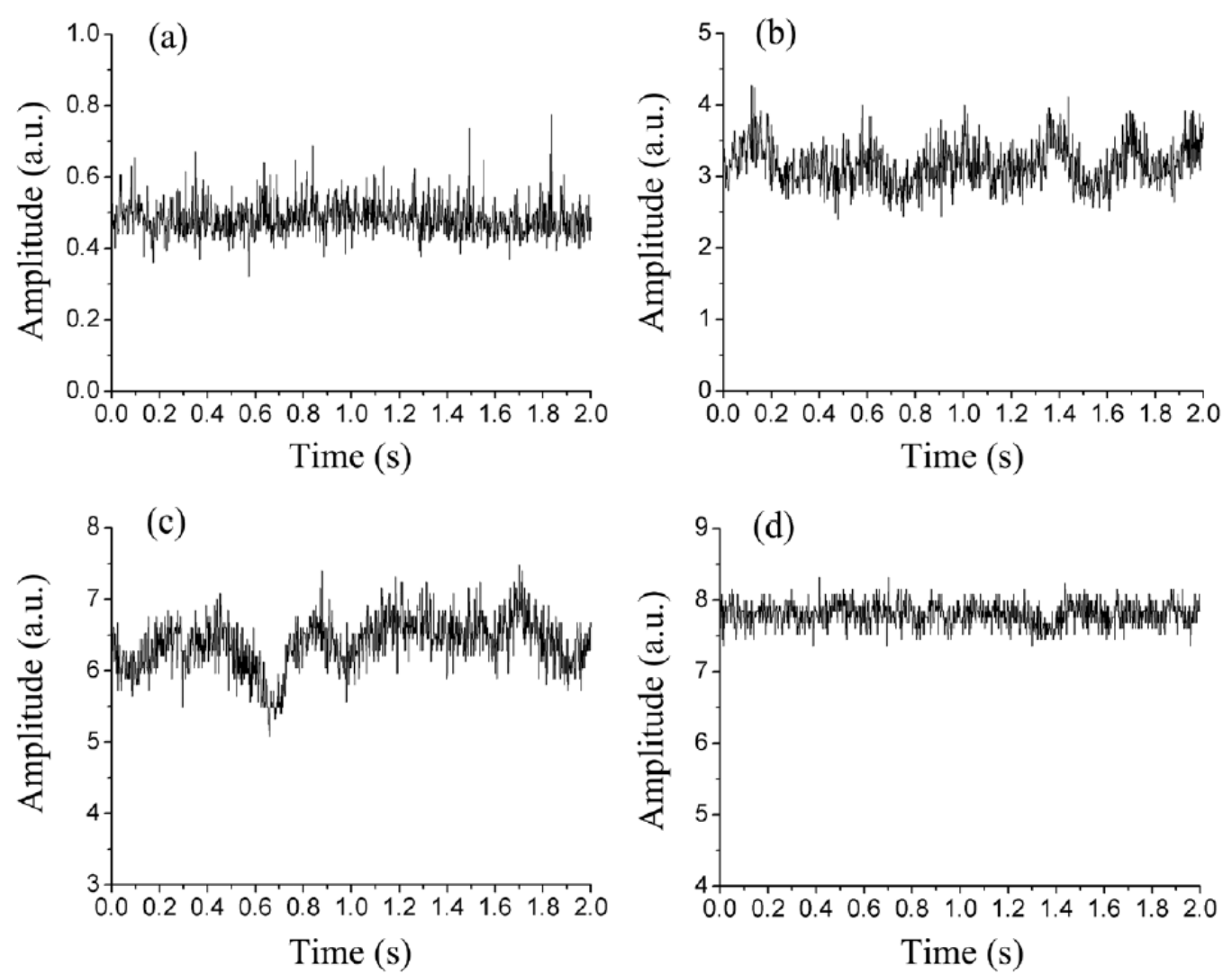

Figure 4.5: Temporal shots of random DFB fiber laser at different pumpings: (a) $49.2 \mathrm{~W}$, (b) $59.2 \mathrm{~W}$, (c) $72.1 \mathrm{~W}$, and (d) $85.6 \mathrm{~W}$ [86].

different gain mechanisms $[12,13,18,22,87-99]$. SBS is one of the alternatives to provide gain for random laser action. Since the generation threshold of SBS is approximately ten times lower than that for SRS, random laser action can start for lower pumping powers $[18,87,88,90,91]$. Also, because the spectral width of SBS gain profile is much narrower than that of SRS, the linewidth of random lasers with SBS gain is hence much narrower. Linewidths as small as $50 \mathrm{~Hz}$ has been reported for SBS-based random DFB fiber lasers when an additional feedback loop was used for further stabilization of laser frequency [18]. A detailed discussion of SBS-based random DFB fiber lasers is given in chapter 6 .

Erbium-doped fiber amplifiers (EDFA) have also been used to generate random lasers [22,92-94]. Low generation threshold are usually observed for EDFA-based random DFB fiber lasers. In [93], an erbium-doped fiber was used as an amplifier in a half-open cavity, with Rayleigh scattering providing coherent feedback. Random laser action was observed with a small generation threshold of $13 \mathrm{~mW}$. By using a tunable band-pass filter (TBPF), random laser action was observed in a narrow bandwidth of $0.04 \mathrm{~nm}$, over a $40 \mathrm{~nm}$ range. This is clearly a benefit of large bandwidth gain mechanisms over narrow gain bandwidths, as is the case for SBS. In another work [94], Zhang and co- 
workers reported a random DFB fiber laser based on a combination of EDFA and Raman amplifiers. The addition of an EDFA in the conventional Ramanbased random fiber laser allow a lower generation threshold, with still high output powers provided by Raman gain.

Although stimulated Rayleigh scattering attracts less attention due to a similar threshold of SBS and lower gain [100], it has been shown to generate random laser [95]. To suppress SBS from the cavity, a fiber with varying core size was used, increasing the SBS threshold by $\sim 7 \mathrm{~dB}$ and precluding its presence in the cavity. Thus, the random DFB fiber laser was uniquely dependent of Rayleigh scattering, since it provides at the same time distributed feedback and light amplification. The work reports a narrow linewidth of $\sim 4 \mathrm{kHz}$, showing that this type of laser could indeed be used in practical applications.

A different concept of light amplification is present when SOAs are used as gain mediums of random DFB fiber lasers. In fiber-based amplification processes, mainly SBS, SRS and stimulated Rayleigh scattering, the amplification is given in a long length of optical fiber. On the other hand, when SOAs are used for optical amplification, the gain is given only along the active region of the semiconductor amplifier, which is usually less than a millimeter. Thus, different than the previous amplification processes, this gain mechanism is said to be localized, which is opposite to the common distributed amplification. Hence, in SOA-based random DFB fiber lasers, the fiber acts only as a passive distributed mirror, thus feedback and gain are spatially decoupled. This pointbased amplification allow simpler models to be built regarding the distributed mirror, otherwise, both light amplification and distributed feedback should be modeled jointly. By separating the feedback from amplification, each effect can be treated isolated, what significantly simplifies the modeling of the physical phenomenon. Therefore, SOA-based random DFB fiber lasers are the focus of this work, and a particular experimental configuration is thoroughly analyzed in chapter 5.

Several works reported on the usage of SOA-based random DFB fiber lasers $[12,13,96-99]$. Although the mode structure was not discussed in detail, single longitudinal mode (SLM) operation with narrow linewidth has been claimed [97-99]. Linewidth measurements might indeed suggest that SOAbased random lasers operate in single mode, with FWHM of the order of a few $\mathrm{kHz}$. Yet, as will be shown in chapter 5 , we provide simulated and experimental evidence that this behavior is a consequence of a nearly continuous multimode operation [12]. 


\section{3 \\ Applications of random lasers}

Random lasers have been reported in multiple applications [8, 10, 71]. Here we discuss four of the most important applications for this class of lasers: imaging, telecommunication, point-based sensing and distributed sensing.

Imaging applications require light sources that are at the same time bright, and with low spatial coherence. These two characteristics are key to an appropriate imaging in strong scattering environments, which are often the case in biological imaging. Bright sources compensate the losses from the scattering environment, and low spatial coherence mitigates the coherent artifacts caused by interference of multiple scatterings. Although lasers and super-luminescent emitting diodes can be designed to be highly bright sources, their high spatial coherence precludes the usage in imaging applications, where light-emitting diodes (LEDs) are preferred. Redding and co-workers have showed that random lasers are specially important for imaging applications, as they can be pumped to provide high brightness, and have naturally low spatial coherence [101].

In an interesting work [102], Saxena and co-workers showed that the Rayleigh feedback in random DFB fiber lasers suppresses noise at high frequencies. This happens because the distributed reflections along the optical fiber have a Lorentzian noise spectrum density, which acts as a frequency-noise filter as it overlaps with the cavity's thermal frequency noise. It was shown that an erbium-doped random DFB fiber laser can achieve an impressive noise level of $6 \mathrm{~Hz}^{2} / \mathrm{Hz}$ at $2 \mathrm{kHz}$, which is even lower than narrow-linewidth erbium-doped fiber lasers. Such low-noise random DFB fiber lasers can find many applications in telecommunications, where low frequency noise sources are of great interest for long distance communications.

Raman-based random DFB fiber lasers were shown to be insensitive to environmental changes because of their wide spectral bandwidth [103]. Making use of this stability, a half open cavity configuration with a point reflector, for instance an FBG, is shown to be a suitable point sensor. Authors have shown that an environmental change at the FBG position translates into a shift of the random laser emitting wavelength. The lasing wavelengths of a 100-km long half-open cavity random laser were experimentally shown to be dependent on three FBGs at 1455, 1560 and $1563 \mathrm{~nm}$. The experiment shows that random DFB fiber lasers can be used in long-distances point-based sensing system. Beyond point-based fiber-optic sensing, random DFB fiber lasers can also be used in a distributed sensing system. In [104], Jia and co-workers showed that a Raman-based random DFB fiber laser can be used in a Brillouin optical time-domain analysis reflectometer to increase the signal to noise ratio (SNR). 
It was achieved a temperature accuracy of $\pm 1{ }^{\circ} \mathrm{C}$, and a spatial resolution of $\pm 2 \mathrm{~m}$. Probably the most remarkable aspect of the work was the high SNR, greater than $50 \mathrm{~dB}$, over $122 \mathrm{~km}$. This experimental result proofs the relevance of random DFB fiber lasers for fiber-optic sensing technology. 


\section{5}

\section{SOA-based random DFB fiber laser}

\section{1}

\section{Theoretical analysis}

In this chapter we make use of the theoretical model for intensity fluctuations of Rayleigh backscattered coherent light developed in section 3.2.2 to analyze the dynamics of an SOA-based random DFB fiber laser. As mentioned before, the usage of an SOA as the gain medium allows us to investigate gain and feedback effects separately, so that the model developed in section 3.2.2 can be used almost as is for analysis of the feedback effect, with only a few modifications being required. Eq. 3-5 was used to calculate the backscattered optical field, but it can be easily modified to calculate the field reflectivity $r$ by simply diving $E_{b}$ by the input optical field amplitude $E_{0}$, i.e.

$$
\begin{gathered}
r(k)=\frac{E_{b}(z=0, k)}{E_{0}}=C A \sum_{i=1}^{N} E_{i}, \\
\text { where } E_{i}=\exp \left(j 2 \phi_{i-1}\right) \int_{-d / 2}^{d / 2} \exp \left(-\alpha\left(l+z_{i}\right)-j 2 \bar{n}_{i} k l\right) d l \\
\text { and } \phi_{i}=\phi_{i-1}+\bar{n}_{i} k d .
\end{gathered}
$$

Different from the analysis given in chapter 3, which was mostly statistical, here we must calculate absolute values for the gain and loss to characterize the laser generation threshold. In this way, the mode field effective area $A$ and the field backscattering coefficient $C$ are no longer irrelevant, and we must use accurate values for proper calculation. For standard single-mode fibers, typical values for $A$ are near $70 \mu \mathrm{m}^{2}$ [54]. To determine the value of the field scattering coefficient $C$, we can calculate the mean backscattered power over a spectral bandwidth large enough to reduce the coherence length to a value much smaller than twice the fiber length. This incoherent backscattered power is given by [54]:

$$
\left\langle\left|E_{b}(z=0, k)\right|^{2}\right\rangle_{k}=S \alpha_{s} E_{0}^{2} \int_{z=0}^{L} \mathrm{e}^{-2 \alpha z} d z
$$

According to [54], typical values for the incoherent power backscatter- 
ing capture coefficient $S$, attenuation scattering coefficient $\alpha_{s}$ and total attenuation coefficient $\alpha$ are $10^{-3}, 3.2 \times 10^{-2} \mathrm{~km}^{-1}$ and $4.6 \times 10^{-2} \mathrm{~km}^{-1}$ (or $0.2 \mathrm{~dB} / \mathrm{km})$, respectively, such that calculation of the right-hand side of Eq. 52 is straightforward for any fiber length $L$. Note that, near $\lambda=1550 \mathrm{~nm}, \alpha$ is approximately equal to $\alpha_{s}$, since the total attenuation is composed of the scattering coefficient $\alpha_{s}$ and an absorption coefficient $\alpha_{a}$. Using $A=70 \mu \mathrm{m}^{2}$ and averaging the left hand side of Eq. 5-2 over a spectral bandwidth of $1 \mathrm{GHz}$ gives $C=2.13 \mu \mathrm{m}^{2}$.

\section{2}

\section{Simulation predictions}

At this point we can simulate the power reflectivity $R(\nu)=\mid E_{b}(z=$ $0, \nu) /\left.E_{0}\right|^{2}$ for any realization of optical fiber, where each fiber realization corresponds to random refractive indexes throughout the fiber - which are constant along fiber sections of $\sim 1 \mathrm{~cm}$. Figure 5.1 displays the simulated power reflectivity for an $8 \mathrm{~km}$-long single-mode fiber within a $5 \mathrm{MHz}$-frequency range around a central frequency of $194.2 \mathrm{THz}(\lambda=1544.86 \mathrm{~nm})$. Similar to results in Figure 3.11, random power fluctuations have peak values reaching $\sim 11 \mathrm{~dB}$ above the $-37.4 \mathrm{~dB}$ mean reflectivity of the $8 \mathrm{~km}$-long single-mode fiber. Note that this mean reflectivity arises from using typical values for the parameters on the right-hand side calculation of Eq. 5-2, which is compatible with the $38 \mathrm{~dB}$ estimated in [98] for an $1 \mathrm{~km}$-long standard single mode fiber.

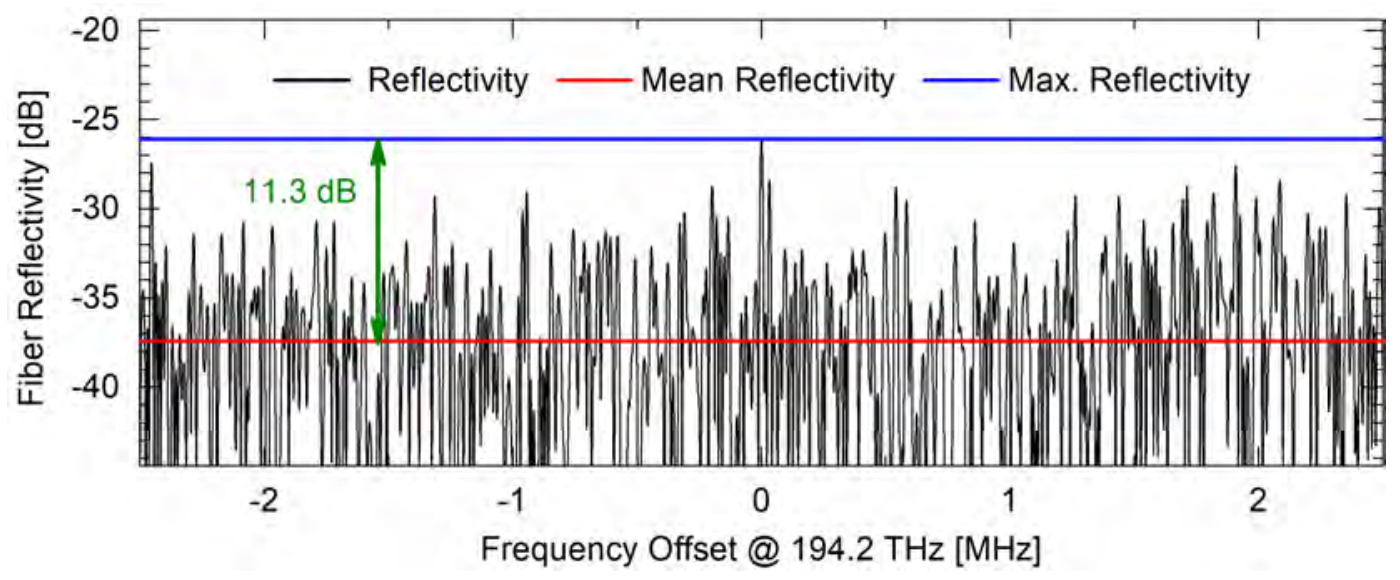

Figure 5.1: Simulated power reflectivity of an $8 \mathrm{~km}$-long standard single mode fiber.

Consider the distributed random feedback laser shown in Figure 5.2, where an SOA acts as the gain medium. The laser cavity is composed by an FBG at one end, and by the distributed Rayleigh backscattering along a single mode fiber of length $L$ at the other end with complex field reflectivity $r$ given by Eq. 5-1. Optical isolators at both ends guarantee that feedbacks are limited 
to the FBG and the fiber. A polarization controller (PC) inside the cavity guarantees that the polarization is maintained along a round-trip, maximizing interference.

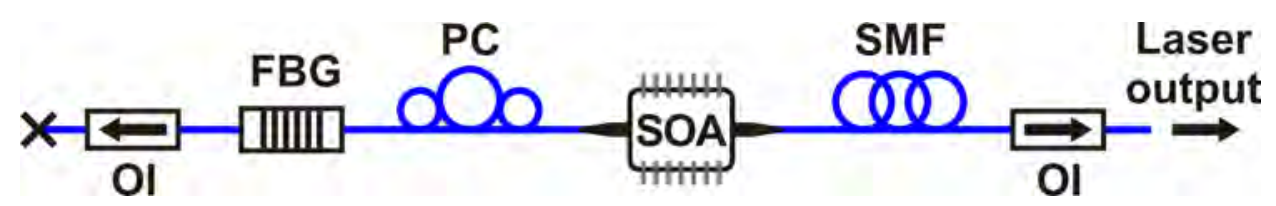

Figure 5.2: Basic scheme of a random feedback fiber laser with localized gain. FBG: Fiber Bragg Grating; OI: optical isolator; SMF: single mode fiber; SOA: semiconductor optical amplifier PC: Polarization Controller.

Having the amplitude and phase of the backscattered optical field, the CW lasing condition can be determined considering the cavity losses and gain, as well as the round-trip phase inside the cavity. Considering the FBG reflectivity $R_{F B G}(\nu)$, the SOA gain $g(\nu)$ and the fiber reflectivity $R_{F i b e r}(\nu)$, the generation threshold condition states (in dB) (see Eq. 2-16):

$$
R_{F B G}(\nu)+R_{\text {Fiber }}(\nu)+2 g(\nu)=0,
$$

where all other losses were neglected as they are indeed very small compared to the low power backscattered from the fiber. Considering the fiber pigtail length $\ell$ between the SOA and the FBG, which adds a frequency independent term to the overall phase (at least in the frequency range considered here), then the phase condition for lasing imposes (see Eq. 2-17):

$$
\Phi_{F B G}(\nu)+\Phi_{F i b e r}(\nu)+\frac{\bar{n}_{\ell} 2 \pi}{\lambda}(2 \ell)=m 2 \pi,
$$

where $\Phi_{F B G}$ and $\Phi_{\text {Fiber }}$ are the phase shifts imparted at the FBG and the long single mode fiber, $m$ is an integer, and $\bar{n}_{\ell}$ is the average refractive index of the fiber section between the FBG and the SOA. For simplicity, we assume here that the reflectivity $R_{F B G}(\nu)$, the gain $g(\nu)$, and the phase shift at the FBG $\Phi_{F B G}(\nu)$ are constant along a wide frequency bandwidth $(\gg 1 \mathrm{GHz})$, such that the frequency dependence of the reflectivity and phase of the random DFB fiber laser are governed by the long single mode fiber.

Given the randomness of $\Phi_{\text {Fiber }}(\nu)$ and $R_{\text {Fiber }}(\nu)$, the frequency of the lasing modes will be randomly spaced within the bandwidth of the Bragg grating. We simulated these fiber parameters over $1 \mathrm{GHz}$ optical bandwidth, which corresponds to the $80 \%$ flat top reflectivity of a $7.5 \mathrm{GHz}$-bandwidth FBG. To analyze the gain and phase conditions, we also considered in the simulation the frequency-independent values $-1 \mathrm{~dB}, \pi$ rad and $2 \mathrm{~m}$ for $R_{F B G}$, $\Phi_{F B G}$ and $\ell$, respectively. Figure 5.3, below, illustrates the simulation result for the cavity loss and round-trip phase within a frequency span of $500 \mathrm{kHz}$ 
centered at the maximum observed reflectivity (minimum loss) given by the reflectivity simulated in Figure 5.1. Each reflection peak is a potential candidate for a mode, provided the phase condition is satisfied. Note that in a uniform feedback cavity such as a Fabry-Perot or a ring fiber laser, the gain is almost frequency independent through the FBG bandwidth, so that only the phase condition defines the mode frequency. In the case depicted in Figure 5.3 we observe that only for the central peak both conditions are satisfied when the cavity gain equals the loss at the central frequency. If the environmental conditions were frozen, the laser threshold would be given by $2 \mathrm{~g} \sim 26 \mathrm{~dB}$ gain needed to match Eq. 5-3, considering that the phase condition is also fulfilled and the phase adjusted to match Eq. 5-4. Hence, CW single mode operation would be obtained near threshold.

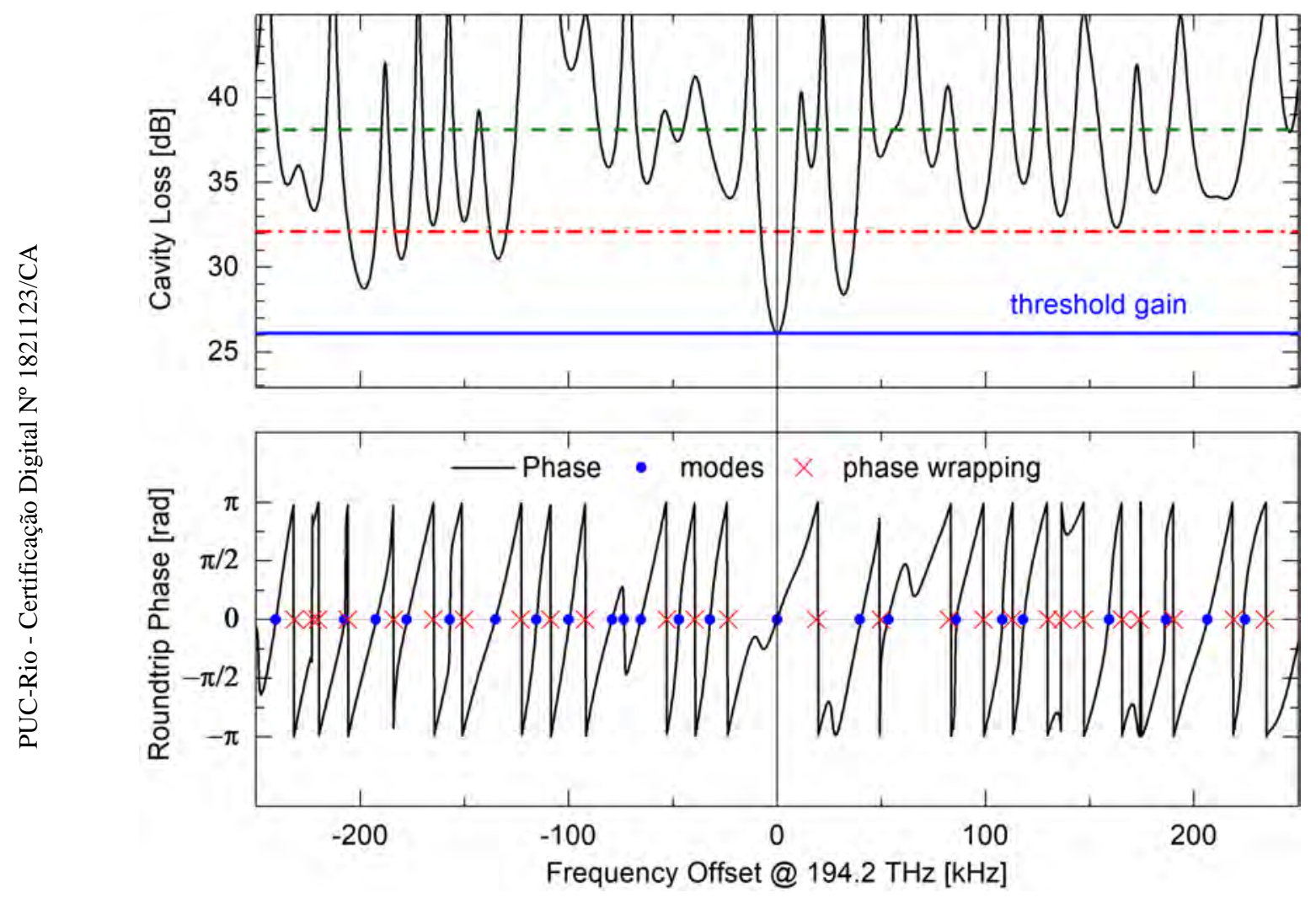

Figure 5.3: Simulated phase of the electromagnetic field after a round trip in a random feedback fiber laser as a function of frequency (offset from $194.2 \mathrm{THz}$ ). Red dash-dot and green dash lines in the top panel represent additional gains of 3 and $6 \mathrm{~dB}$ provided by a single pass on the SOA. Red crosses in the bottom panel do not represent modes -- they are due to the phase wrapping procedure. Blue points correspond to real solutions of the phase condition.

At higher gains, more reflectivity peaks can satisfy the gain condition and more modes are simultaneously allowed, provided the corresponding phase condition is also satisfied. In the example depicted here, limited to $500 \mathrm{kHz}$ from the 7.5 GHz bandwidth available for lasing, increasing the SOA gain by 
$3 \mathrm{~dB}$ above the threshold (red dash-dot line in Figure 5.3), a total gain of $6 \mathrm{~dB}$ is added to the system and seven modes would eventually be lasing if the phase condition were matched. If the gain is increased by additional $3 \mathrm{~dB}$ (green dash line in Figure 5.3), many modes will be enabled at the same time because the gain condition would be relaxed. Therefore, at low SOA gains, modes are defined mainly by the gain condition, but as the gain increases, the gain condition becomes less restrictive so that at high gain the phase condition actually defines the lasing modes. Therefore, for SOA currents high enough, unevenly spaced multimode operation should be observed.

Under laboratory conditions, tiny fluctuations in temperature, stress and/or birefringence force the power reflectivity $R$ to be a time-dependent random function $R(t)$, so that reflectivity peaks in Figure 5.1 are expected to fluctuate in time, similarly to a scintillation phenomenon. Moreover, $\Phi_{\text {Fiber }}$ and $\ell$ are time dependent so that CW operation of the laser is unfeasible because phase and gain conditions must be simultaneously satisfied. As the SOA gain is increased, a phase-matched scintillation peak can eventually reduce the cavity loss below twice the SOA gain, so that a laser pulse builds up as in a passive Q-switched laser randomly driven by the environmental conditions. The phase in km-long single mode fibers in laboratory environment has been already reported to vary in ms time scales $[67,105]$ and we expect the scintillation peaks to vary in a similar way. Hence, lasing should start with Q-switched milliseconds-long pulses as the SOA gain is increased.

Because of the random nature of the cavity loss, lasing threshold needs to be defined within a statistical framework. A few approaches have been reported to determine the threshold of random lasers, including a transition from Gaussian intensity distribution to Levy distribution $[79,106,107]$, and the observation of replica symmetry breaking [108-111]. Here we show that very low cavity loss events can appear, generating random laser pulses with very low probability. By making use of the ergodicity validation presented in section 3.4, then the statistics of reflectivity fluctuations at a given frequency over time are equal to to those over all frequencies at a given time. Hence, we calculated the statistics of the reflectivity from five runs of our model simulations over 1 $\mathrm{GHz}$ bandwidth, in order to increase the statistical reliability of low probability events. Figure 5.4(a) displays the cumulative probability of cavity loss events smaller than a given value. We observe an increasing slope as the loss decreases below $30 \mathrm{~dB}$, suggesting a lower bound near $26 \mathrm{~dB}$. Hence, as the SOA gain increases, the random laser will start generating single mode pulses when the SOA single pass gain approaches $13 \mathrm{~dB}$ (double pass gain $=26 \mathrm{~dB}$ ), which could be defined as a lasing threshold. Further increase in SOA gain the pulse 
rate increases until several modes will be lasing at the same time.

To evaluate randomness of the mode spacing we calculated the mode separation from the phase condition, which is usually the one used for mode calculation, considering high enough gain. The statistics of the frequency spacing between adjacent (consecutive) modes of the simulated $8 \mathrm{~km}$ random feedback fiber laser is presented in Figure 5.4(b). The average value of mode spacing was found to be $26.5 \mathrm{kHz}$, close to the evenly spaced value of a $4 \mathrm{~km}$ long Fabry Perot cavity. This is close to the value that would be obtained by direct integration of Eq. 3-3 with uniform fiber parameters. This figure shows that when many modes are allowed, their beat noise will cover all frequencies in a featureless white spectrum instead of the series of equally spaced tones that would be observed in a uniform fiber.
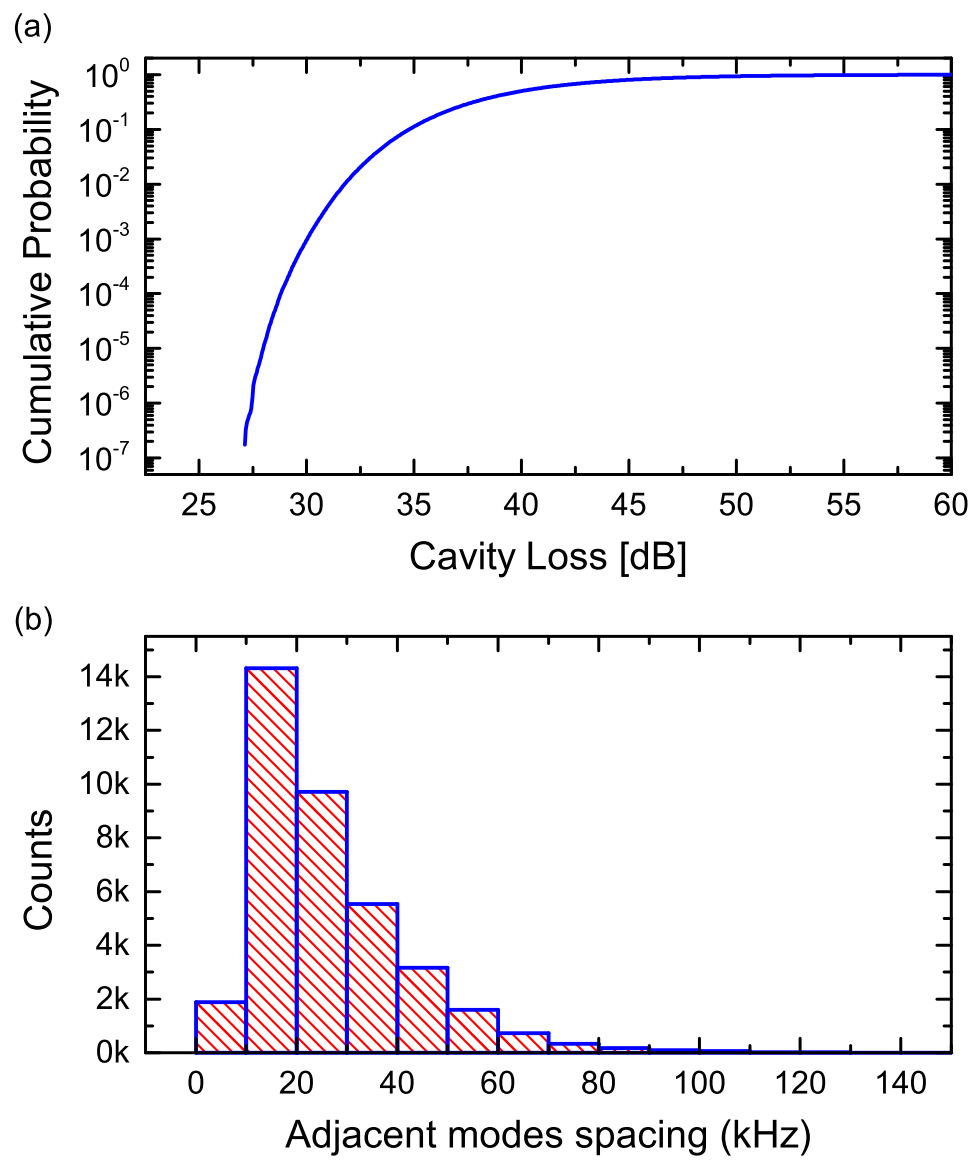

Figure 5.4: (a) Simulated cumulative probability of instantaneous cavity loss. (b) Histogram of adjacent modes spacing found for simulated $8 \mathrm{~km}$-long random feedback fiber laser within $1 \mathrm{GHz}$ bandwidth. The average mode spacing of $26.5 \mathrm{kHz}$ is close to the $25.7 \mathrm{kHz}$ value from a uniform feedback fiber laser. 


\section{3 \\ Experimental setup}

The experimental setup is presented in Figure 5.5. Light is generated by an SOA, and the cavity is formed by a tunable FBG with $7.5 \mathrm{GHz}$ bandwidth and by the Rayleigh backscattered light from an $8 \mathrm{~km}$-long dispersion shifted fiber (DSF). The Rayleigh incoherent backscattered power coefficient from this DSF fiber was measured with OTDR to be $3 \mathrm{~dB}$ greater than that of a standard SM fiber, which is in good accordance with theoretical calculation [54]. The FBG acts as both a fixed mirror and a tunable filter with $7.5 \mathrm{GHz} 3 \mathrm{~dB}-$ bandwidth, sharp enough to filter Brillouin scattered light out of the cavity. A polarization controller was inserted in the cavity to optimize interference effects. Optical isolators at both ends guarantee that undesired reflections are eliminated.

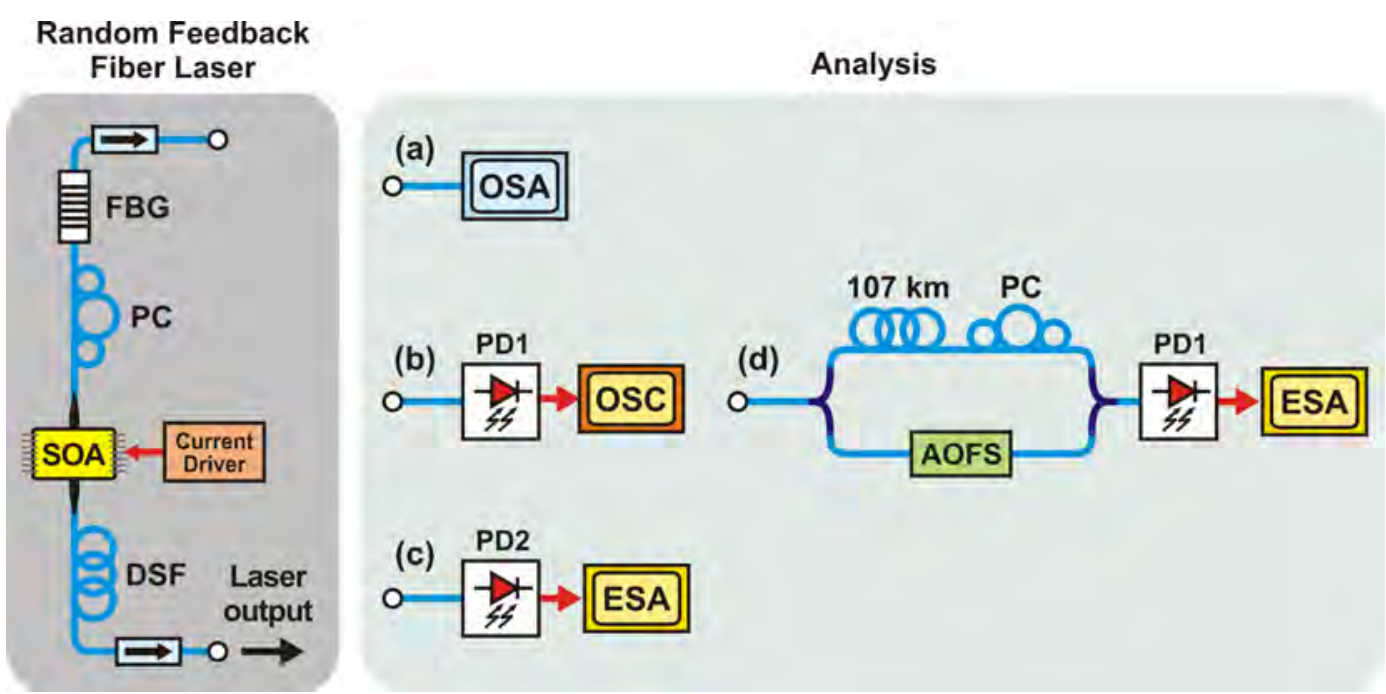

Figure 5.5: Experimental setup of Random Feedback Fiber Laser (left) and measurement setups (right). AOFS: Acousto-Optical Frequency Shifter. DSF: Dispersion Shifted Fiber; ESA: Electrical Spectrum Analyser; FBG: Fiber Bragg Grating; OSA: Optical Spectrum Analyser (High and Low resolutions); OSC: Oscilloscope; PC: Polarization Controller; PD: Photodiode.

The characteristics of the random laser light were analyzed as follows. High resolution optical spectral analysis was performed with a $0.16 \mathrm{pm}$ resolution optical spectrum analyzer (OSA) with low $(<\mathrm{Hz})$ video bandwidth (VBW), while fast optical measurements were performed with a $0.03 \mathrm{~nm}$ resolution OSA with VBW of $10 \mathrm{kHz}$ - both represented as OSA in Figure 5.5(a). Time-domain measurements were performed with a $3.5 \mathrm{GHz}-$ bandwidth oscilloscope and a $125 \mathrm{MHz}$-bandwidth amplified photodiode (PD1) - Figure 5.5(b). Direct output noise spectral measurements were performed with an electrical spectrum analyzer and a 5 GHz-bandwidth photodiode 
(PD2) - Figure 5.5(c). Self heterodyne linewidth measurements were performed with a $107 \mathrm{~km}$ unbalanced Mach-Zehnder interferometer (MZI), an acousto-optic frequency shifter and a polarization controller, in a delayed selfheterodyne scheme - Figure 5.5(d).

\section{4 \\ Results and discussions}

\section{4 .1}

\section{Output power}

Analysis of the laser output optical spectrum in the $0.03 \mathrm{~nm}$-resolution OSA shows that, when low currents drive the SOA, only the ASE spectrum is observed. Figure 5.6(a) displays how the averaged optical power at the central wavelength of the FBG varies with SOA driving current. We observe that above $\sim 200 \mathrm{~mA}$ the average output power starts increasing with the driving current, as a typical threshold in a current driven laser. The SOA gain curve presented in Figure 2.15 is replicated in Figure 5.6(b) for convenience, from which we infer that the threshold behavior occurs when the SOA gain reaches $11.2 \mathrm{~dB}$. Due to the higher reflectivity coefficient of the dispersion shifted fiber, $3 \mathrm{~dB}$ should be added to the reflectivity simulations in section 5.2 , so that the model predicts that the laser should start firing pulses near a gain close to $11.5 \mathrm{~dB}$, in a remarkable agreement between theoretical model and experiment.

As expected from the simulation, near the generation threshold current $I_{T H}$, strong laser peaks are observed in both OSC and OSA, as shown in Figures 5.7(a) and (e), respectively. Because the OSA used in these measurements was a sweeping wavelength one, its $10 \mathrm{kHz}$ video bandwidth was able to detect ms-long pulses. It should also be noted that the OSA limited to $0.03 \mathrm{~nm}$ resolution determines when a pulse took place rather than its real wavelength. Peak powers up to $\sim 30 \mathrm{~dB}$ above the ASE power are observed, confirming that whenever a Rayleigh scintillation peak is high enough to overcome the gain condition a Q-switched pulse is fired. These peaks are observed as $\sim 1 \mathrm{~ms}-$ long pulses (inset of Figure 5.7(a)), arising at random times in a small rate. As the current starts increasing, the pulse rate also increases, but the maximum pulse peak power remains relatively stable. One might think that the pulse peak power is limited by the single pass stimulated Brillouin scattering, but we calculated the theoretical SBS threshold power by $P_{t h} \sim 21 A /\left(g_{b} L\right)$, with $A$ for the DSF equal to $50 \mu \mathrm{m}^{2}, L=8.47 \mathrm{~km}$ and $g_{b}$, the Brillouin gain for silica fibers, equal to $5 \times 10^{-11} \mathrm{~m} / \mathrm{W}$, showing a threshold power of $\sim 2.63 \mathrm{~mW}$, which is much higher than the pulses' peak power shown in Figures 5.7(e)-(g). 

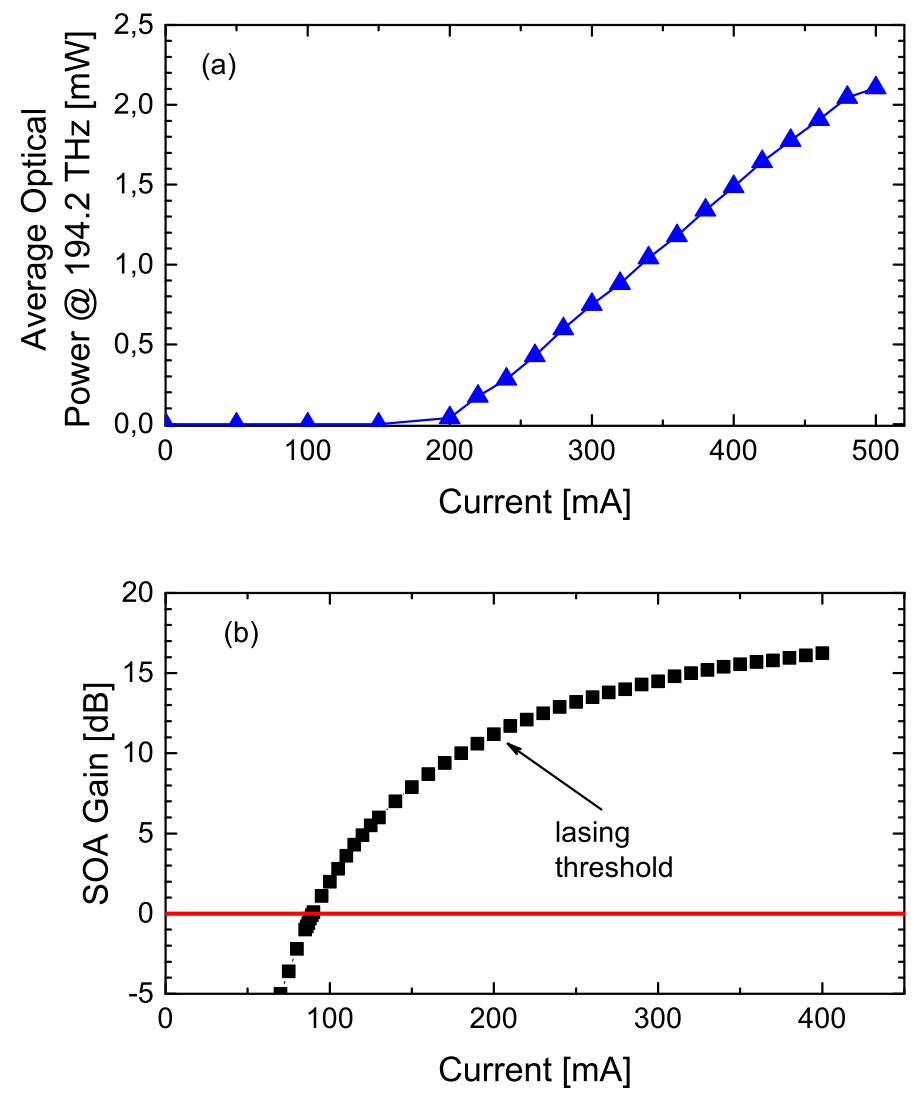

Figure 5.6: Variation of the random laser average optical power with SOA driving current for an $8 \mathrm{~km}$-long dispersion shifted fiber (a). SOA end to end gain curve (b).

A similar result is obtained in [98], showing that SBS is not present in the laser cavity for low currents.

Further increase in the driving current eventually lead to pulse overlapping. This is shown in Figures 5.7(b) and (c), which present the laser output for driving currents of $205 \mathrm{~mA}$ and $210 \mathrm{~mA}$, respectively, with correspondent optical spectra shown in Figures 5.7(f) and (g). At these current levels, the averaged optical power increase is due to the increasing pulse rate, rather than by an increasing pulse power. 


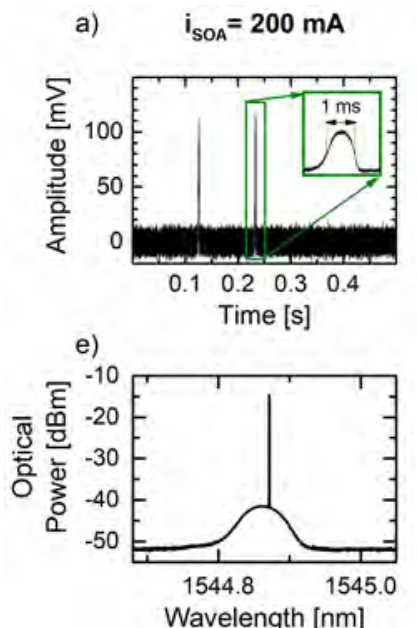

b) $i_{\text {SOA }}=205 \mathrm{~mA}$

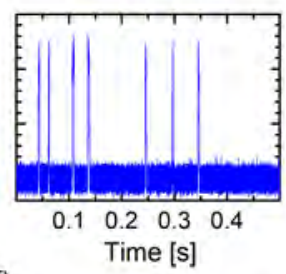

f)

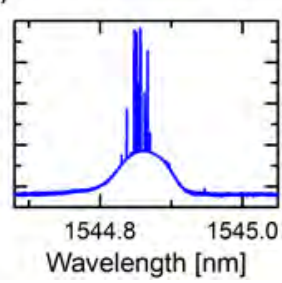

c) $\mathrm{i}_{\mathrm{SOA}}=210 \mathrm{~mA}$

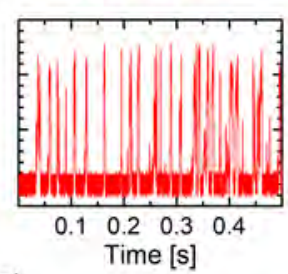

g)

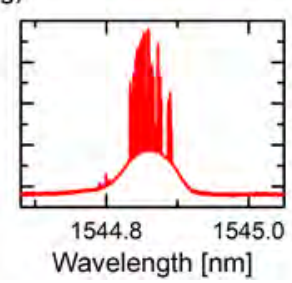

d) $\mathrm{i}_{\mathrm{SOA}}=400 \mathrm{~mA}$

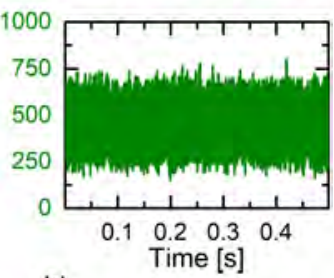

h)

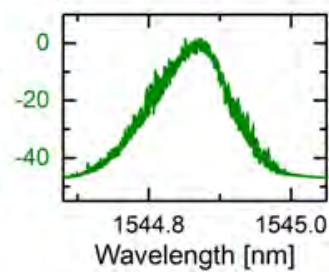

Figure 5.7: Detected pulses emitted by random lasers at SOA driving currents

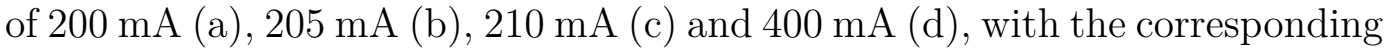
optical spectra in (e), (f), (g) and (h), respectively.

\section{Why do random DFB fiber lasers emit light pulses?}

Although the word "random" in random DFB fiber lasers refers to the disorder of the feedback mechanism promoted by multiple scattering centers, the temporal emission of random DFB fiber lasers is also random, in the sense that light pulses are emitted at random times. This behavior is observed when the laser is pumped near the generation threshold, whereas under high pumping, light pulses arise continuously, in a CW-like operation. The origin of random emission in time is the feedback mechanism, i.e., Rayleigh backscattering.

In random DFB fiber lasers, single-mode fibers act as special mirrors, with reflectivity highly fluctuating with optical frequency. This fluctuation is an interference effect, caused by the contribution of multiple Rayleigh scattering centers throughout the optical fiber. Because of environmental changes along the fiber, which alter the fiber's refractive index, the reflectivity profile is dynamic, varying in time. Hence, the reflectivity fluctuates similar to a scintillation phenomenon. Eventually, a very high reflectivity peak can arise, which translates into a sudden drop of the cavity's loss. Provided that the laser has enough gain to overcome the losses and that the phase condition is satisfied, laser action starts - analogous to a Q-switch laser. However, since the reflectivity fluctuates, the high reflectivity peak is sustained only for a short time, such that the cavity loss rapidly increases, breaking the gain condition for lasing. Thus, light pulses are randomly emitted with durations governed by fiber's phase fluctuation period, which is of the order of milliseconds for km-long fibers. 


\section{4 .2 \\ Mode operation}

The random emission of laser pulses shown in Figure 5.7 in both time and optical-frequency domain is precisely what was expected from the model description and simulations. Indeed, near threshold, very few modes have peak reflectivity high enough to reach the lasing condition in both phase and amplitude. When a scintillation peak from the fiber promotes Q-switching, pulsed laser action starts. After a while, variations in both the pigtail phase and $8 \mathrm{~km}$-long fiber drive the cavity out of the phase condition and the laser power drops out. The duration of the optical pulses near threshold can be associated to a single mode with a lifetime of $\sim 1 \mathrm{~ms}$. When the SOA gain is much further increased, many modes are satisfying the lasing conditions at the same time, giving rise to continuously overlapping pulses. This condition can be seen in Figures 5.7(d) and (h) for $400 \mathrm{~mA}$ driving current.

As pulses start overlapping a strongly fluctuating output power is observed. An overlap of emitting pulses is shown in Figure 5.8, where frequency modes overlap for a driving current of $210 \mathrm{~mA}$. A zoom-in on the right portion of the overlapping pulses shows evidence of two strong beat notes. It is important to highlight that, in order to acquire oscilloscope data over several milliseconds, which is required for analyzing pulses overlap with typical $1 \mathrm{~ms}$ duration, the resolution bandwidth of the oscilloscope had to be reduced from 3.5 GHz to $10 \mathrm{MHz}$. Thus, mode beating could only be observed for very close modes, distant from each other by no more than $5 \mathrm{MHz}$, due to Nyquist limit. The electrical beating tones shown in Figure 5.8, which come from the beating of optical modes, can be observed as a subcarrier frequency of $4.92 \mathrm{MHz}$ modulated in amplitude by $151 \mathrm{kHz}$. These frequencies build up at the same time, around $14.5 \mathrm{~ms}$, so we can infer that three optical modes are beating. It happens as follows: two modes, apart from each other by $\Delta \nu=9.84 \mathrm{MHz}$, emerge at $13.75 \mathrm{~ms}$, when no beating is observed due to the low resolution bandwidth set on OSC. At $14.5 \mathrm{~ms}$, a third mode arises, with optical frequency in between of the first two modes, $5.07 \mathrm{MHz}$ apart from one of them, and $4.77 \mathrm{MHz}$ from the other. Electric beating of these two frequencies results in the observed $4.92 \mathrm{MHz}$ and $151 \mathrm{kHz}$, corresponding to the half-sum and half-difference of $5.07 \mathrm{MHz}$ and $4.77 \mathrm{MHz}$, respectively.

\section{4 .3}

\section{Spectral analysis}

As shown in the previous section, when the current is increased multimode behavior dominates the laser output, and multimode beating, such as 


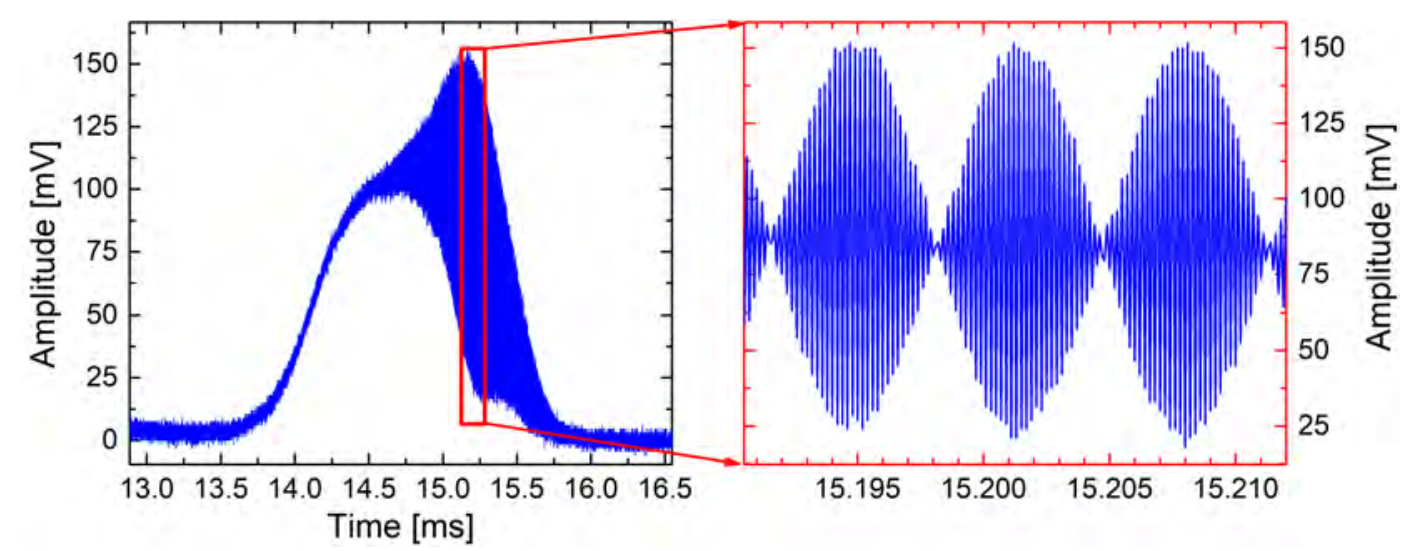

Figure 5.8: Output of random laser showing three overlapping pulses, corresponding to the overlap of three simultaneous modes. A zoom-in on the red rectangle area shows evidence of strong beat notes.

the one displayed in Figure 5.8, should occur at all frequencies within the FBG bandwidth, as shown in Figure 5.7(h), resulting in a noisy output as displayed in Figure 5.7(d). The output laser spectrum was then measured with the high-resolution OSA, while the detected power spectrum was measured with a 5 GHz-bandwidth detector. Figures 5.9(a) and (b) display these two measurements for a driving current of $500 \mathrm{~mA}$, together with a comparison between the calculated and measured electric power spectrum. The measured electric spectrum shown in Figure 5.9(b) indeed exhibits the floor spectrum extending to the FBG bandwidth, confirming that the floor power in the self-heterodyne spectral measurements is due to multimode beating noise, as will be mentioned below. Further confirmation is given by the comparison between the measured output power spectrum and the expected electric spectrum obtained by the self-convolution of the measured optical spectrum in Figures 5.9(b). It should be noted that the discrepancy between the calculated and measured power spectrum above $5 \mathrm{GHz}$ is due to the limited bandwidth of the detector used in the experiment. The agreement between these measurements supports the statement that multimode operation occurs at high currents and multimode beating noise dominates the output spectrum. In addition, this experimental conclusion complies with the simulation result provided in Figure 5.4(b), where a random mode spacing was achieved, thereby mode beating should continuously extend within the FBG bandwidth.

The linewidth of the random laser was measured with the delayed selfheterodyne technique by using an MZI unbalanced by $535 \mu$ s and an AOFS, which shifts the optical frequency in one arm of the MZI by $86.83 \mathrm{MHz}-$ Figure5.5(d). As typical durations of light pulses are around $1 \mathrm{~ms}$ (see inset of Figure 5.7(a)), the MZI delay is short enough to insure a minimum $~ 50 \%$ overlap of a single pulse whilst still long enough to guarantee the Wiener- 

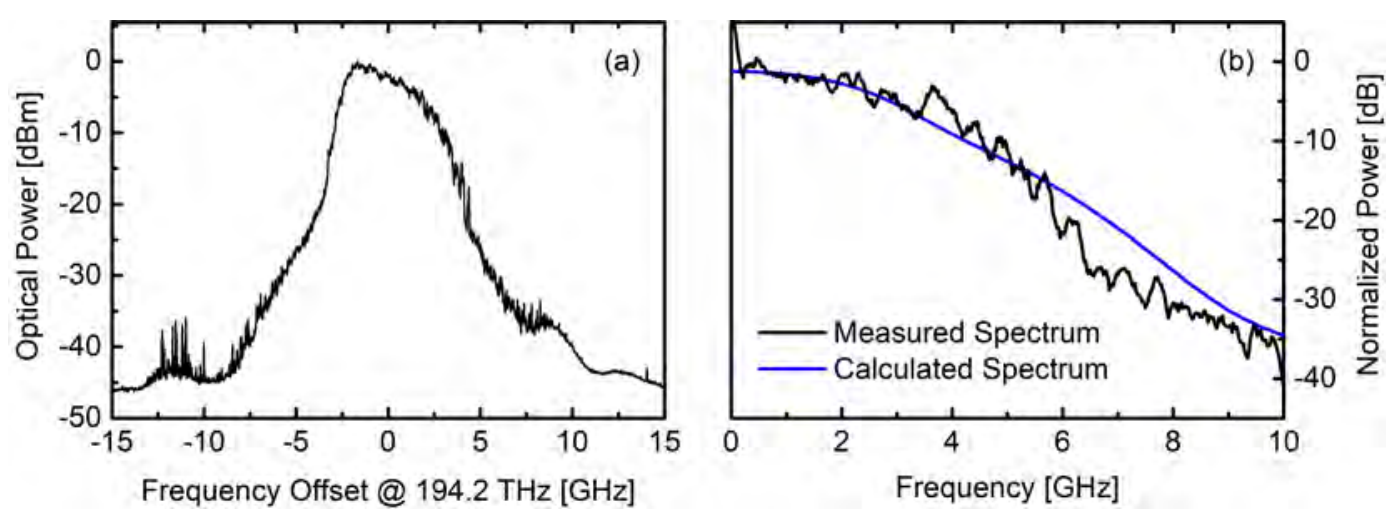

Figure 5.9: (a) Random laser optical spectrum measured in a high resolution (0.16 pm) OSA; (b) Measured and calculated electrical power spectrum. Both measured curves were obtained when driving the SOA with $500 \mathrm{~mA}$.

Khintchin condition for $\sim \mathrm{kHz}$ linewidth measurements [54]. Figure 5.10(a) displays the linewidth measurement results at seven different SOA driving currents. Each curve was obtained by averaging 100 measured spectra at the ESA. As the random laser only presents single mode operation near the threshold, then, to measure the linewidth of an individual lasing mode, the SOA driving current must be set to $I_{T H}$. However, the pulse rate is extremely low at $I_{T H}$, and the mode spectrum falls below the noise floor of the spectrum analyzer. Linewidth measurements could be observed for driving currents greater than $278 \mathrm{~mA}$, when self-mode beating arose centered at the AOFS shifting frequency. At such current, the $3 \mathrm{~dB}$-linewidth was found to be $4 \mathrm{kHz}$, and it increases with SOA gain up to $7 \mathrm{kHz}$. Thus, we can infer that the mode linewidth is at most $4 \mathrm{kHz}$, which agrees with the calculated width of reflectivity peaks $(<10 \mathrm{kHz})$ obtained from Figure 5.1. In addition, such narrow linewidth corresponds to a coherence length greater than $20 \mathrm{~km}$, which is in accordance with the theoretical model that assumed a coherence length greater than twice the optical fiber length.

a)

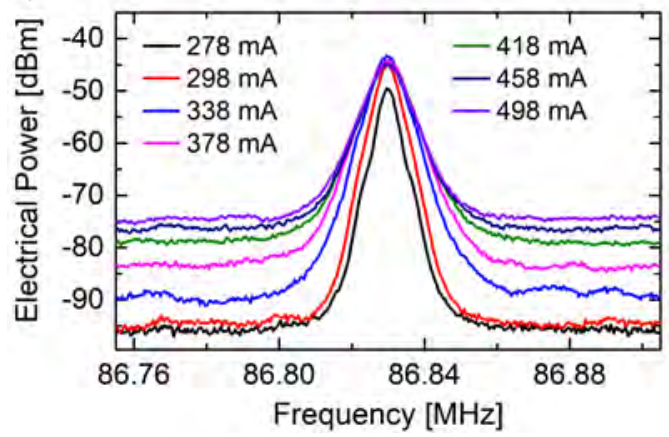

b)

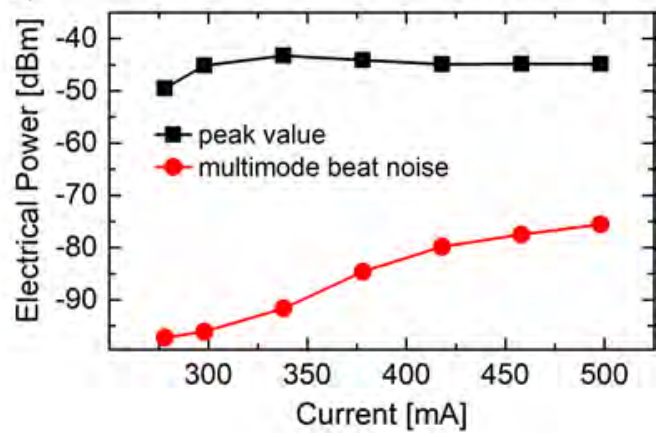

Figure 5.10: (a) Random laser linewidth for different SOA driving currents; (b) Intensity of the mode mean power and multimode beat noise floor. 
At currents higher than $278 \mathrm{~mA}$ several modes are beating to form the measured spectra. While the self-beat of each mode gives rise to the mode peak at $86.83 \mathrm{MHz}$, multimode beat will generate the floor level observed in Figure 5.10(a). The intensity of the self-beat note slightly increases for driving currents up to $338 \mathrm{~mA}$, where saturation is observed. On the other hand, the floor level keeps increasing further on, as shown in Figure 5.10(b). In agreement with the behavior pictured in Figures 5.6(a) and 5.7, we see that the initial increase in the average power is due to the increase in the frequency of laser pulses, which maintain their peak power relatively constant. The increase in the floor power, also observed in $[97,98]$, is due to the increase in the multimode population in the laser output. As demonstrated in Figure 5.9, the floor level extends to the FBG bandwidth, but the measurements of Figure 5.10 were limited by the $125 \mathrm{MHz}$-bandwidth detector used in this high resolution measurement.

An important feature of the spectral measurements in Figure 5.10 is that the measured power of the self-beating signal saturates with the SOA current, whereas the multimode floor keeps increasing, indicating that more coexisting modes are present in the laser output. Since there are more modes in the output, the self-beating note should increase correspondingly, unless the mode power decreases accordingly. This experiment supports the conclusion that at high currents mode power diminishes and is limited by mode competition, whereas near threshold it is relatively constant.

Mode competition also affects mode's linewidth. As shown in Figure 5.10(a), the linewidth widens from 4 to $7 \mathrm{kHz}$ when the SOA driving current is increased from 278 to $498 \mathrm{~mA}$. Near the lasing threshold, in the absence of mode competition, mode's lifetime is of approximately $1 \mathrm{~ms}$. In a scenario with gain competition, a lasing mode may be annihilated when a new mode rises in the cavity. The new mode consumes part of the available gain, which may break the gain condition of the previously lasing mode. Thus, the presence of more modes in the laser cavity results in a reduction of mode's lifetime, which is observed as an increase in the measured linewidth. Note that, the direct measurement of mode's lifetime is not possible at high gain as can be observed in Figure 5.7(d), such that this behavior can only be indirectly measured in the ESA. 


\section{6 \\ Brillouin-based random DFB fiber laser}

\section{1 \\ Introduction}

In the previous chapter, the longitudinal mode dynamics of an SOA-based random DFB fiber laser was discussed. As mentioned in section 4.2.3, RFLs have been developed with multiple gain mechanisms, and it is thus important to analyze if the characteristics of mode dynamics observed for RFLs with point-based gain also hold for distributed gain. Among the distributed gain mechanisms, SBS is of particular interest for two reasons. First, since the generation threshold of SBS is approximately ten times lower than that for SRS, random laser action can start for very low pumping powers ( $\sim$ few $\mathrm{mW})$. And second, because the spectral width of SBS gain profile is much narrower than that of SRS, SOA and fiber-amplifiers, the linewidth of random lasers with SBS gain is hence order of magnitudes narrower. In fact, the mode's linewidth is still much narrower than the SBS gain spectral width because of a cooperation between SBS and Rayleigh scattering [23].

Although different configurations have been used to generate RFL with Brillouin gain, the mode structure has not been investigated in detail. In [87], authors reported the generation of random lasing by separating Brillouin gain and Rayleigh scattering in two different fibers, and observed narrow spikes on the top of the Brillouin gain spectrum, suggesting a single-mode operation in a preferred wavelength selected by Rayleigh scattering. In chapter 5 we showed that similar spikes are observed in SOA-based RFLs, and could mislead to the conclusion of single-mode operation. However, supported by theoretical and experimental results, we demonstrated that single-mode operation is only possible in pulsed regime near the lasing threshold, while multimode operation governs lasing emission under high pumping. It might be thought that the different gain properties of SOA and SBS gains could lead to a different mode structure and dynamics for random lasers based in such gain mechanisms. In this chapter, we experimentally investigate the mode dynamics of Brillouinbased random fiber lasers by using a general but simple configuration, similar to that reported in [89], where both gain and feedback are distributed in the 
same fiber.

\section{2}

\section{Experimental setup}

The experimental setup is shown in Figure 6.1. The random lasing process is initiated by a single mode pump laser-diode emitting CW light at $1547.63 \mathrm{~nm}$, which is amplified with an Erbium-doped fiber amplifier (EDFA). A variable optical attenuator (VOA) and a 99:1 beam splitter are used for power monitoring purposes at tapping point $\mathrm{A}$, with $99 \%$ of light being directed to a tunable FBG. The FBG is tuned to be transparent at the pumping wavelength, such that light at 1547,63 $\mathrm{nm}$ is fully transmitted to an 8-km long dispersion shifted fiber. When high intensity light is sent to the fiber, acoustic waves are thermally excited $[112,113]$, resulting in a periodic modulation of the fiber's refractive index, which in turn scatters light through Bragg diffraction - a process known as Brillouin scattering. The scattered light undergoes a Doppler frequency shift proportional to the acoustic wave velocity, giving a

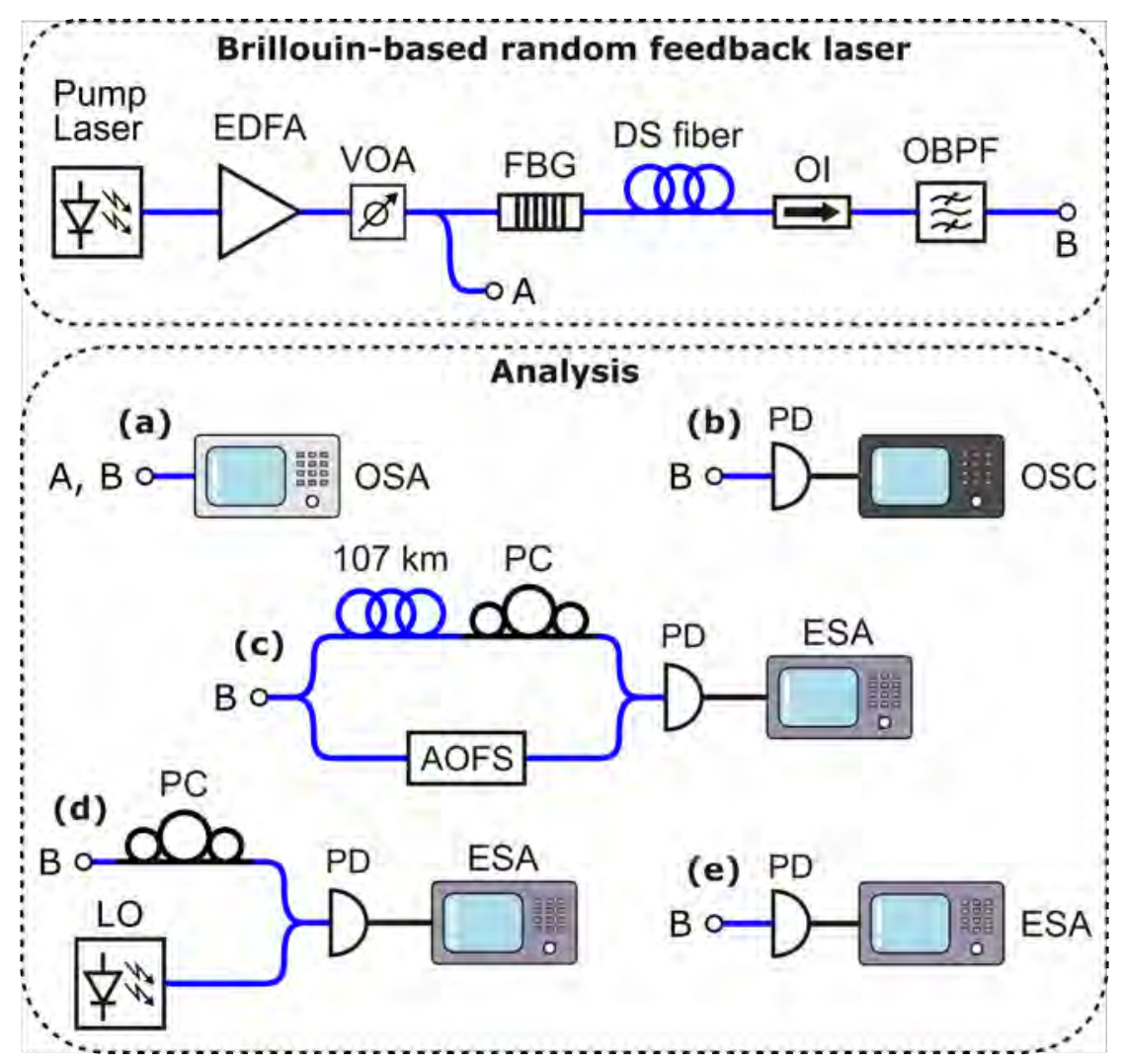

Figure 6.1: Experimental setup. AOFS: Acousto-optic frequency shifter; DS: Dispersion shifted; EDFA: Erbium-doped fiber amplifier; ESA: Electrical spectrum analyzer; FBG: Fiber Bragg grating; LO: Local Oscillator; OBPF: Optical band-pass filter; OI: Optical isolator; OSA: Optical spectrum analyzer; OSC: Oscilloscope; PC: Polarization controller; PD: Photodector; VOA: Variable optical attenuator. 
Brillouin backscattered Stokes light down-shifted by $10.9 \mathrm{GHz}$ at $1550 \mathrm{~nm}$, and with a spectral width of the order of $\mathrm{MHz}$ [114]. If the light intensity is high enough to reinforce the acoustic wave, the process is said to be stimulated, i.e., SBS, which provides gain within its spectral width.

We tuned the FBG to match the Stokes component of the Brillouin light. The FBG's narrow bandwidth of $0.06 \mathrm{~nm}(\sim 7.5 \mathrm{GHz})$ makes it transparent to the pumping wavelength, while reflecting the Stokes light back into the fiber. This reflected light propagates along the fiber and is, itself, backscattered through Rayleigh scattering, which is an elastic phenomenon preserving the frequency of the incident light. Thus, the RFL comprises a linear half-open cavity composed of an FBG at one end and randomly distributed Rayleigh scattering at the other end, with gain provided by SBS. An optical isolator at the fiber end guarantees that undesired reflections are precluded. In order to analyze strictly the properties of the RFL at its lasing wavelength, a tunable optical band-pass filter is placed at the RFL output, with spectral width of $0.03 \mathrm{~nm}$, and tuned to match the Brillouin scattered Stokes light, rejecting the remaining pump light and residual ASE from the EDFA. Note that the ASE light coming from the EDFA at the Brillouin gain spectral region is rejected at the input FBG, not disturbing the gain regime of the RFL.

Spectral measurements were performed with a high resolution $(20 \mathrm{MHz})$ optical spectrum analyzer in Figure 6.1(a) whereas time domain measurements were performed with a $125 \mathrm{MHz}$-bandwidth pre-amplified detector and a 1 GHz-bandwidth oscilloscope in Figure 6.1(b). Ultimate resolution spectral measurements were performed either with the self-heterodyne technique, Figure 6.1(c), or with heterodyne detection, Figure 6.1(d). Laser light spectral content was directly measured with an electrical spectrum analyzer, Figure $6.1(\mathrm{e})$.

\section{3}

\section{Results and discussions}

Figure 6.2(a) displays the optical spectra measured at the laser output (point B in Figure 6.1) with the high-resolution optical spectrum analyzer (Figure 6.1(a)) under two conditions: with and without the OBPF. A $50 \mathrm{~dB}$ rejection of the remaining pumping laser light is observed with the OBPF. We removed the filter to measure the relation of output pump power at $1547.63 \mathrm{~nm}$ and Stokes light power at $1547.71 \mathrm{~nm}$ as a function of input pump power. The result is shown in Figure 6.2(b), indicating a typical laser regime after a threshold of $\sim 3.5 \mathrm{~mW}$. A thorough analysis of power distribution of a similar RFL is given in [89], confirming that the power increase at Brillouin 
wavelength, $\lambda_{B}$, indeed corresponds to laser action, and not simply to SBS. The low generation threshold obtained in our case is due to the use of a DSF, which has a smaller core and initiates SBS at lower powers compared to standard fibers.
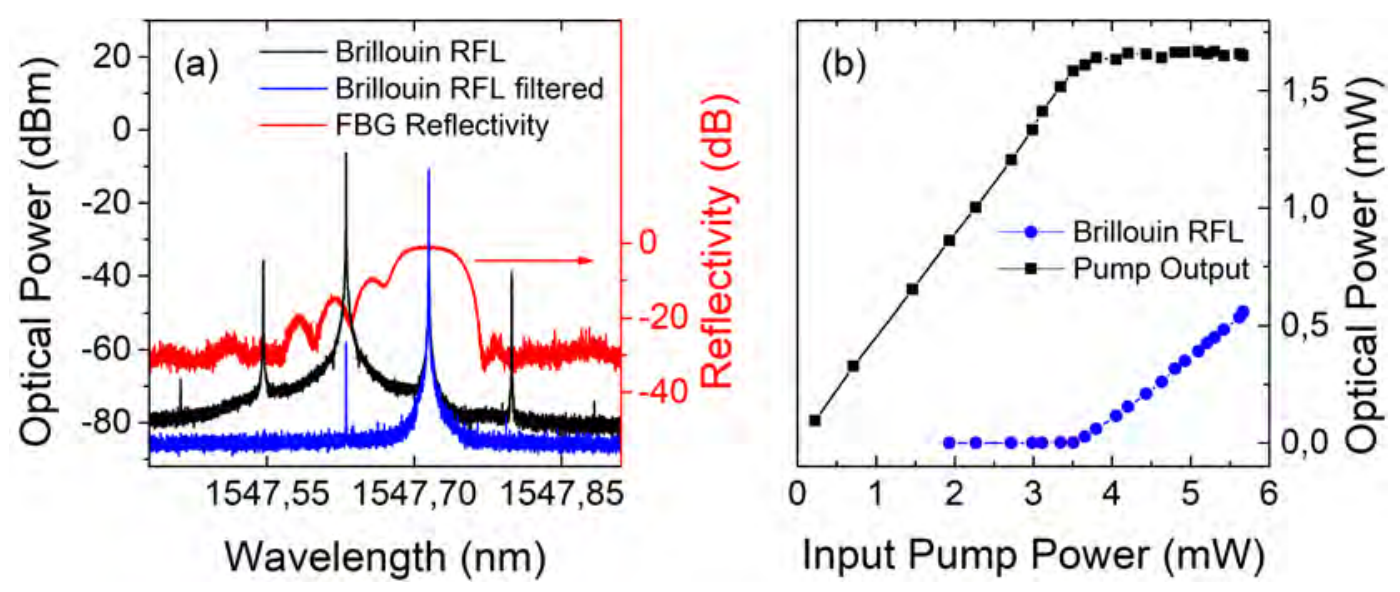

Figure 6.2: (a) Brillouin-based RFL output spectrum before and after spectral filtering, and FBG reflectivity profile. (b) Power dependence of Brillouin RFL and output pump laser with input pump power.

As demonstrated in chapter 3, Rayleigh backscattering in optical fibers can be treated as a low reflectivity mirror $(<0.01 \%)$ that is highly frequency selective. Narrow reflectivity peaks were shown to reach high values above the mean reflectivity (> $11 \mathrm{~dB}$ - see 3.11 ). Because of environmental changes, such as temperature variations and vibrations, the Rayleigh backscattering reflectivity profile fluctuates in time, such that there is a probability associated with the rise of high reflectivity peak. In the linear cavity show in Figure 6.1, high reflectivity peaks translate into valleys in the cavity loss. If this loss decreases to values lower than the SBS gain, and provided that the phase condition is satisfied, then $\mathrm{CW}$ laser action should initiate. However, since reflectivity peaks depend on the fiber's state, i.e. on the refractive index longitudinal profile, which is randomly defined by environmental changes, these peaks are sustained only during short time intervals, of the order of milliseconds for kilometer-long fibers $[67,105]$. As a result, laser action should be observed in a pulsed regime and at narrow frequency modes when pumping near the generation threshold. In fact, in the same way described in section 5.2, the generation threshold should be defined in a statistical framework, as low probability reflectivity peaks could result in laser action at very low pumping.

For experimental validation, we measured the RFL output with the $125 \mathrm{MHz}$-photodetector, and observed the detected signal at the $1 \mathrm{GHz}-$ bandwidth oscilloscope, as shown in Figure 6.1(b). The measurement result is shown in Figure 6.3(a). As expected, near the generation threshold, optical 
pulses with durations between $400 \mu$ s and $1 \mathrm{~ms}$ are observed. A typical laser pulse is shown in Figure 6.3(b), corresponding to the lasing of a single mode defined by the Rayleigh scattering reflectivity profile. Increasing the pumping slightly above the threshold, at $3.7 \mathrm{~mW}$, strong beat notes are observed for some lasing pulses, as shown in Figure 6.3(c). This frequency beating is a result of the rise of two simultaneous lasing modes. When the pumping increases above the threshold, the gain condition for lasing is more easily satisfied, and less intense reflectivity peaks, with higher probability, turns to be candidates to start laser action. These candidates are available throughout the SBS spectral width, which we measured to be of $8 \mathrm{MHz}$. Hence, since the bandwidth of our detection scheme is $125 \mathrm{MHz}$, the simultaneous lasing of any two modes is always observed as a beating tone. Thus, the oscilloscope trace shown in Figure 6.3(a) for a pumping of $3.5 \mathrm{~mW}$ (red curve) reveals definitely single mode pulses, as no beating is observed. Note that, even though single mode operation is certain for each pulse, the lasing wavelengths are unknown, and can in fact be different for each pulse.
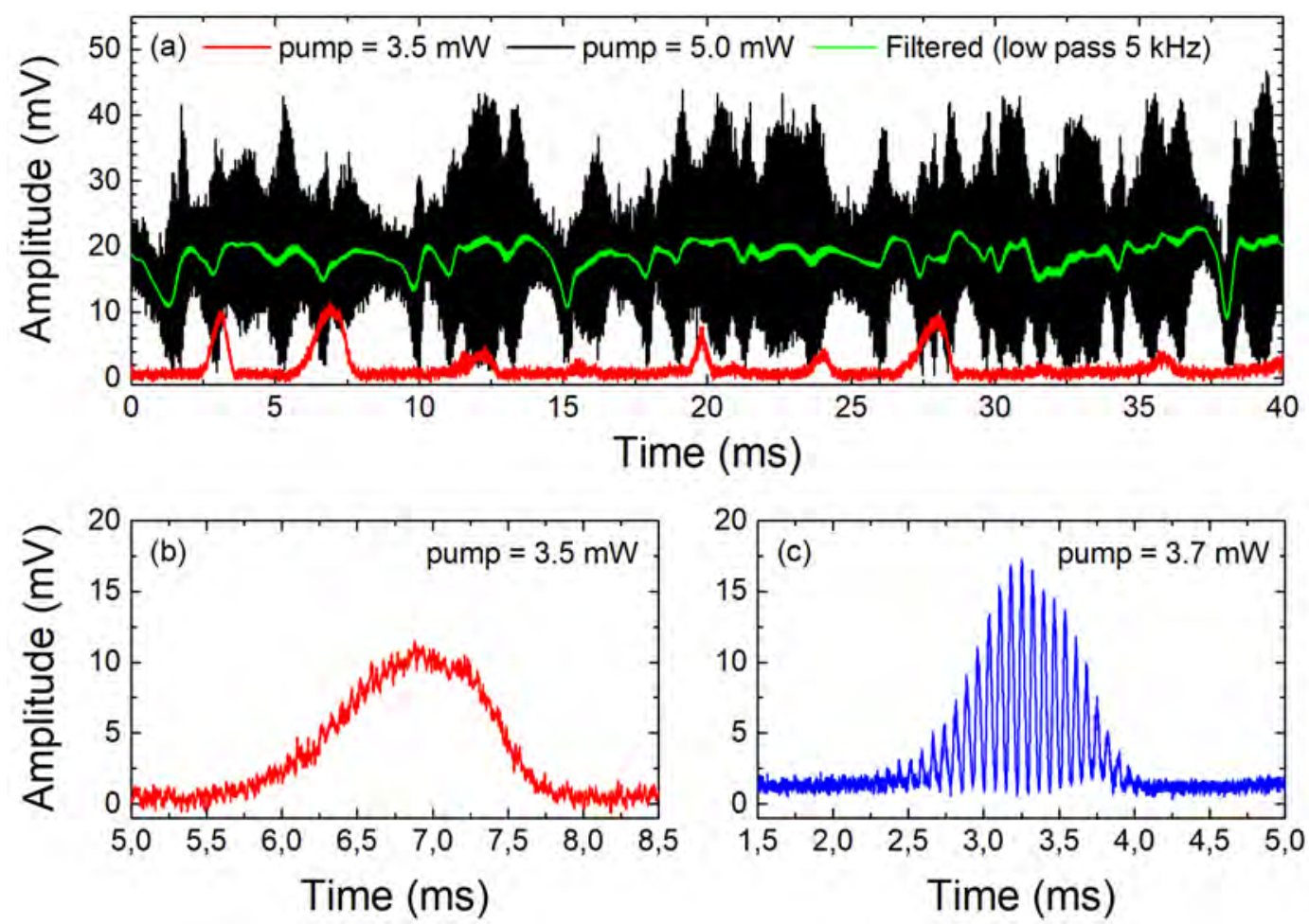

Figure 6.3: RFL output measured at two pumping powers (a). Typical laser pulses emitted when pumping near (b) and slightly above (c) the generation threshold.

Increasing the pumping power, the gain condition is further relaxed, and many modes within the SBS gain bandwidth start lasing simultaneously giving rise to fast intensity fluctuations due to mode beating. This case is exemplified in the black curve shown in Figure 6.3(a), obtained when pumping with 
$5.0 \mathrm{~mW}$. The mean power increase is evident when compared to the emission near the threshold, justifying the power increase shown in Figure 6.2(b). A significant average output power fluctuation is still observed though, which cannot be related to mode beating. Indeed, the average mode spacing of an $8 \mathrm{~km}$-long fiber linear open cavity is $25 \mathrm{kHz}$ (see Figure 5.4(b)), with mode separation starting at $\sim 5 \mathrm{kHz}$ so that fluctuations slower than that must rather be attributed to fluctuations in the Rayleigh reflectivity modulating cavity losses. The green curve in Figure 6.3(a) is the result obtained by filtering the laser output intensity with a $5 \mathrm{kHz}$ low pass filter showing that the average output power fluctuates at a rate compatible with Rayleigh scattering intensity fluctuations.

A spectral analysis of lasing modes is performed with configurations shown in Figure 6.1(c-e). First, we used the delayed self-heterodyne technique to measure the laser spectral content as shown in Figure 6.1(c). The RFL output is split into two arms by a 50:50 beam splitter. Light in the bottom arm is frequency shifted by $88.97 \mathrm{MHz}$ at an acousto-optic frequency shifter, and it interferes at a beam combiner with a delayed copy of the laser's output that traveled in the upper arm. We used a delay line of $107 \mathrm{~km}$, allowing linewidth measurements as low as $\sim 1 \mathrm{kHz}$ [54]. A polarization controller maximizes the interference, which is detected at the $125-\mathrm{MHz}$ bandwidth photodetector and measured at an ESA. Results are shown in Figure 6.4(a) for different pumping powers.

Similar to [88] and [87], a narrow linewidth spike of $6 \mathrm{kHz} @ 3 \mathrm{~dB}$ is observed on the top of a noise pedestal, indicating an optical spectral width of $3 \mathrm{kHz}$ due to a convolution effect. Although it may seem that the narrow spike can be attributed to a preferred mode defined by the narrow reflectivity spikes of Rayleigh scattering, this is not actually true. Since the selfheterodyne method involves the beating between the RFL and its frequencyshifted copy at $88.97 \mathrm{MHz}$, any narrow linewidth lasing mode beats with itself at precisely the shifted frequency. Thus, the narrow spike observed is an artifact of the measurement technique, representing the simultaneous lasing of narrow linewidth modes at different frequencies within the SBS gain bandwidth. This conclusion becomes more evident when analyzing the noise pedestal behavior. Although the spectral width of the noise pedestal indeed coincides with that of SBS gain, its increase with pumping power does not correspond simply to an increase in the available gain. As more gain is available in the cavity, multiple narrow linewidth modes can satisfy the lasing condition. While the beating of any mode with itself gives a narrow peak at $88.97 \mathrm{MHz}$, the beating between any two different and simultaneous modes will give a frequency tone falling 
in the SBS gain spectral width. In this way, the noise pedestal is actually a continuous beating between multiple lasing modes, i.e., a multimode beating spectrum. The multimode beating power and the lasing spike peak power are plotted as function of the input pump power in Figure 6.4(b), complying with theoretical expectations. In addition, this result is also compatible with the result shown in Figure 6.3(c), where a strong beat note was observed.
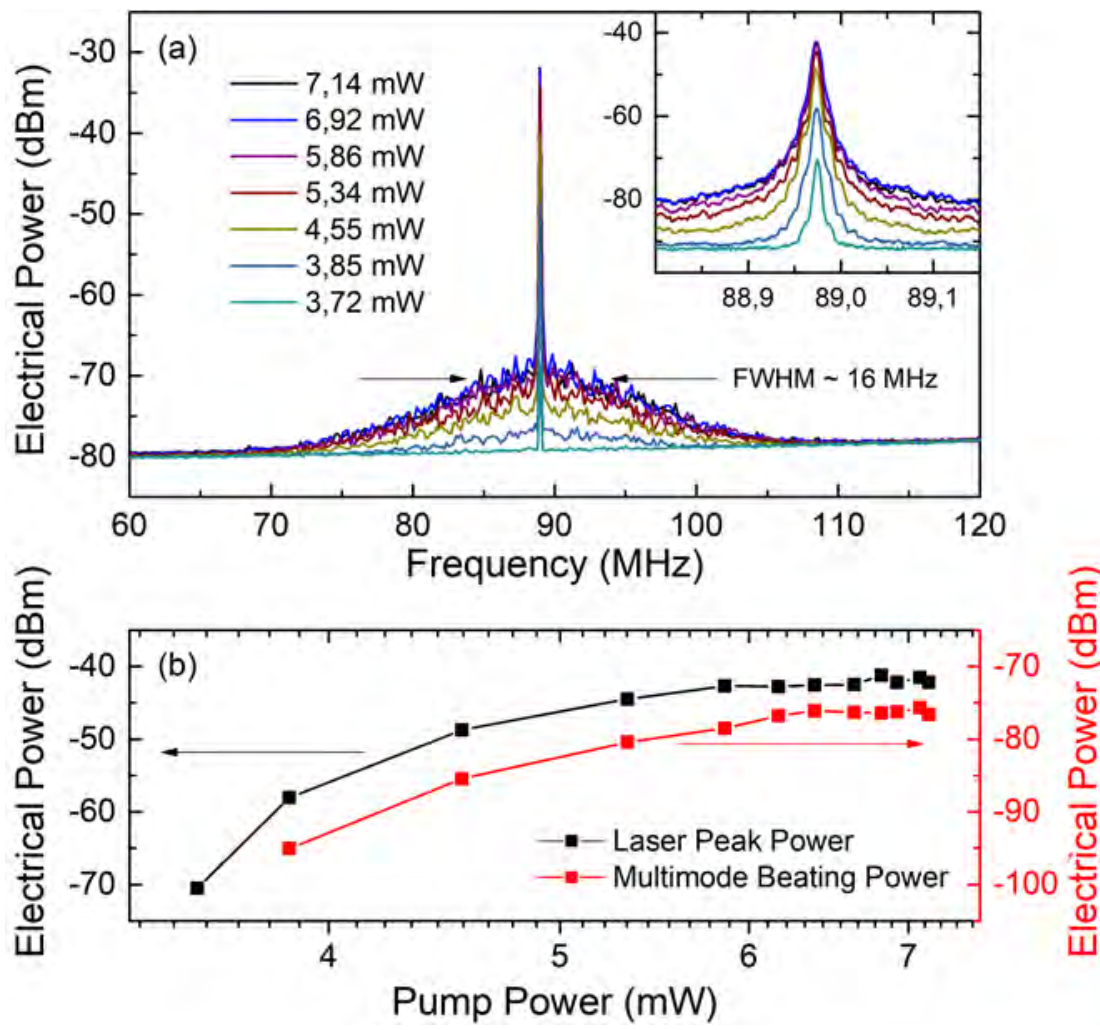

Figure 6.4: (a) Measurement of RFL spectral content with the delayed selfheterodyne technique for different pumping powers. (b) Peak power increase, and multimode beating power increase with pumping power.

To measure the RFL linewidth without the self-beating artifact of delayed self-heterodyne measurements, we conducted a heterodyne measurement with a local oscillator as shown in Figure 6.1(d). The LO used was an external cavity tunable laser source with $3 \mathrm{~dB}$-linewidth of $100 \mathrm{kHz}$. Thus, the experimental configuration limits linewidth measurements to $100 \mathrm{kHz}$. We tuned the LO's optical frequency to a value close to the laser frequency $\nu_{B}$ (or $\lambda_{B}$ ) such that their beating falls within the $125 \mathrm{MHz}$ bandwidth of the photodetector, and its output is read at an ESA. The measurement result is shown in Figure 6.5(a) for a pumping of $6 \mathrm{~mW}$. As expected, no narrow linewidth spike is observed on the top of the multimode beating spectrum. This is a significant result, definitely proofing that the Brillouin-based RFL operates in a continuous multimode regime when pumped above the generation threshold. Mode linewidth is narrow $(\sim 3 \mathrm{kHz})$, but above threshold many modes are randomly emitted within the 
SBS gain spectral width so that the RFL's effective linewidth equals the SBS bandwidth.

We used a direct detection scheme to further evaluate the spectral content of the laser light. The experimental configuration is shown in Figure 6.1(e), where the RFL output is detected directly with the PD, and the frequency content measured at an ESA. Multimode emission will result in a beating signal from simultaneously emitted modes, whose frequency covers the SBS gain bandwidth. Pump power was kept at $6 \mathrm{~mW}$, and the measurement result is shown in Figure 6.5(b). The measured spectrum exhibits a 3-dB width of $\sim 8 \mathrm{MHz}$, agreeing with the RFL linewidth observed in the heterodyne method, and with the result shown in Figure 6.4(a), where the 3-dB bandwidth of the multimode beating contribution is doubled due to a convolution effect.
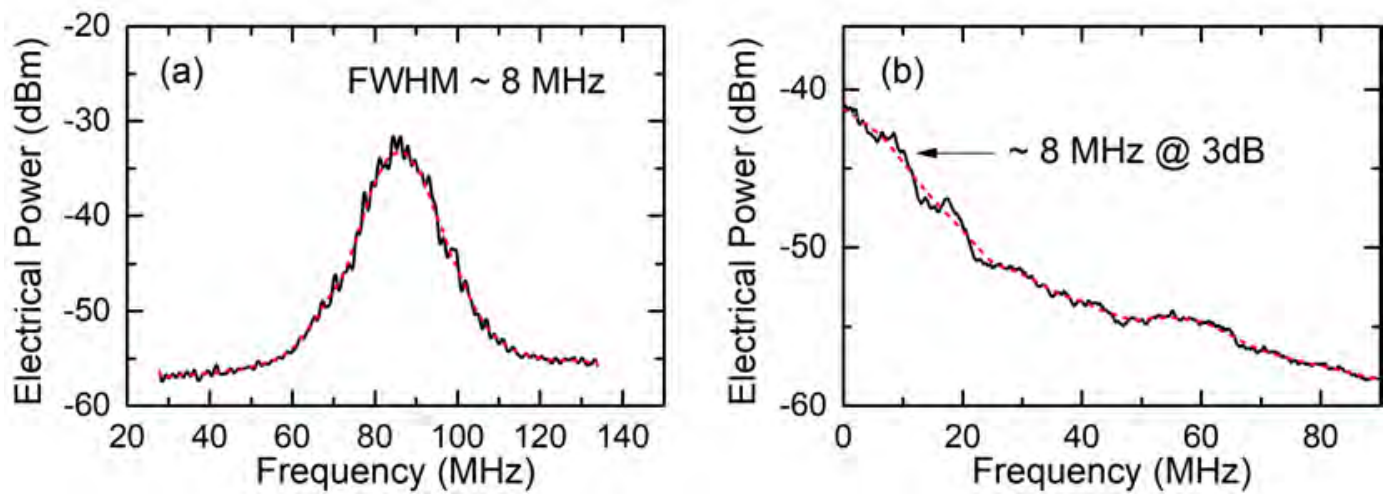

Figure 6.5: (a) Heterodyne measurement with local oscillator of Brillouin-based RFL. (b) Direct detection of RFL output.

Two interesting conclusions are taken when comparing the experimental results described above with those from chapter 5 for an SOA-based RFL. First, although the gain mechanisms differ in many levels, the build-up of lasing modes proceeds in a similar fashion, indicating that near the threshold the fluctuation of Rayleigh backscattering reflectivity profile governs lasing action. Second, since the wide spectral gain of the SOA-based RFL was filtered with a $7.5 \mathrm{GHz}$ FBG, lasing modes were observed throughout $\sim 7.5 \mathrm{GHz}$. Mode competition in such wide band was shown to limit the mode lasing power and to widen the linewidth, corresponding to a reduction in photons lifetime in a laser cavity mode due to mode competition. In the Brillouin RFL under analysis, even though a tunable FBG with the same bandwidth was employed, the farthest frequency difference between two lasing modes was limited to $\sim 8 \mathrm{MHz}$ due to the much narrower bandwidth of the gain profile. Thus, modes are available in a spectral band 3 orders of magnitude narrower than that of the SOA-based RFL. With much less modes competing for gain, mode competition did not limit the mode power, which can be seen 
in Figure 6.4(b) as the multimode beating power increases in the same way as the mode lasing power, and in Figure 6.4(a) as the linewidth is unaffected with high pumping. However, both laser peak power and multimode beating power exhibit a saturation at an input power around $5.5 \mathrm{~mW}$. At this power level, the second order Brillouin light is observed, strongly indicating that it is responsible for gain saturation.

At this point, the understanding of mode dynamics of both SOA-based and Brillouin-based RFLs allows us to analyze the average power fluctuations observed from a broader perspective. By comparing results shown in Figures $6.3(\mathrm{a})$ and $5.7(\mathrm{~d})$, it is noted that under high pumping the low frequency fluctuations $(<5 \mathrm{kHz})$ of the Brillouin RFL are much more significant than those of the SOA RFL. The latter exhibits a clear high frequency fluctuation given the multimode beating across nearly $7.5 \mathrm{GHz}$, but its average power remains practically constant. The average fluctuation is directly related to the gain bandwidth available for lasing. Supported by theoretical results shown in Figures 3.3(a) and 3.6(a), the average Rayleigh backscattering intensity is highly stable when wide optical frequency bandwidths are considered, whereas it can fluctuate severely in narrow bands. In other words, the availability of lasing modes is so high in wide frequency bandwidths that under high pumpings there will always be multiple modes lasing and sustaining a stable average power. The opposite occurs for narrow gain bandwidths, for instance, the SBS gain bandwidth, where lasing modes are available throughout a bandwidth orders of magnitude smaller. This conclusion is consistent with experimental results reported in the literature. As shown in [89], the Brillouin-based RFL analyzed exhibit clearly a low frequency fluctuation, being related with the narrow gain bandwidth of SBS. A much wider gain bandwidth is available when Raman-based RFL are explored, where a highly stable average power should be seen. Indeed, as reported in [1], the random DFB fiber laser studied showed to be highly stable under high pumping. In fact, in this case the gain bandwidth is so wide $(\sim 10 \mathrm{~nm})$ that a large number of modes beat at the same frequencies with random phases, averaging out even the high frequency modulation given by multimode beating, and low relative intensity noise (RIN) laser light can be generated. 


\section{7}

\section{Breaking phase and gain conditions}

\section{1 \\ Introduction}

As mentioned in chapter 1.1, the complexity in spatial distribution of three and two-dimensions random lasers is reduced when 1D systems are used $[9,115]$. Optical fibers naturally provide transverse confinement of light, so the light beam generated by a random fiber laser is nearly Gaussian [10]. However, as discussed in the previous chapter, spectral and temporal characteristics are still far more complex than those of conventional lasers. Many works reported techniques to control spectral behavior of random fiber lasers $[14,96,116]$. Given the simple integration of random fiber lasers with fiber-coupled off-the-shelf optical devices, a straightforward approach to tune the emission wavelength of RFL is to use spectral filtering, which is often implemented with Fabry-Perot filters or FBGs. In [14], a tunable optical band-pass filter (TOBPF) was introduced in the conventional random DFB fiber laser setup, with Raman gain, and tunable emission was experimentally observed in the range $1535-1570 \mathrm{~nm}$. The tuning range is similar to those obtained in traditional Raman fiber lasers [117-119]. However, the tunable random DFB fiber laser showed a far better power flatness throughout the tuning range, not exceeding 3\%, while traditional Raman fiber lasers are typically $20 \%$ flat in nearly the same tuning range [14].

Another approach for spectral selectivity in RFL is the usage of random FBGs [96]. Specially fabricated FBGs with distributed random phase errors promote random distributed feedback similar to Rayleigh backscattering, and it has been shown to be an efficient feedback mechanism for random lasing. In [116], authors fabricated a $30 \mathrm{~cm}$-long random FBG made from hydrogenated Erbium-doped polarization-maintaining fiber and experimentally obtained random lasing at $1534.5 \mathrm{~nm}$ with a narrow spectral width of $0.5 \mathrm{pm}$. By easily modifying the manufacturing process of the random FBG, spectral tuning can be achieved within the gain bandwidth.

Beyond spectral tuning, it is also of great interest to control the temporal emission of random fiber lasers. Clearly, as discussed in sections 4.2.2 and 5.4.1, 
this control is not possible when pumping near the generation threshold, since light pulses are randomly emitted under such condition. However, at high pumping random DFB fiber lasers continuously emit light, which can be tailored with internal or external modulators. The usage of external modulators, such as conventional electro-optic modulators (EOM), to shape the amplitude or phase of the random DFB fiber laser output light proceeds in the same way as in any conventional laser, as the laser properties are not altered by such devices. On the other hand, internal modulators, i.e. modulators placed inside the cavity (or open-cavity), change the conditions required to sustain laser action, thus being a relevant matter of study for random DFB fiber lasers.

Internal modulation of random DFB fiber lasers was first proposed in [120]. Bravo and co-workers demonstrated random laser action in a cavity composed of a dispersion compensating fiber (DCF) and a modulated mirror structure. The latter was implemented with the usage of an optical circulator, a polarization controller and an EOM. The light backscattered in the DCF is directed to the mirror structure, which acts as an on/off reflector when the EOM is modulated with electric pulses. The frequency and temporal width of the electric pulses selects a section of the DCF to contribute with distributed feedback. Although the work shows an interesting configuration of internally modulated random DFB fiber laser, authors did not discuss the build up of laser action, which should be directly related to the frequency driving the EOM. A similar work, with a much more detailed discussion about laser action is provided in [121]. Instead of an EOM device, it was used an SOA acting as optical switch. Gain was provided by both the SOA and an Erbium-doped fiber, which also promotes random distributed feedback. Authors showed that laser oscillation can be obtained for modulation frequencies from several $\mathrm{kHz}$ to a few MHz.

In this chapter, we make use of intra-cavity phase modulation in the SOA-based random DFB fiber laser configuration presented in chapter 5 to investigate the effects on gain and phase conditions for lasing. The experimental setup is presented in section 7.2. Next, in section 7.3 it is provided a theoretical analysis of intra-cavity phase modulation effects in phase and gain conditions for random lasing. Section 7.4 provides experimental results and discussions. It is important to mention that all experimental results presented in this chapter were obtained using an SOA-based RFL, but similar results were observed for the Brillouin-based RFL discussed in chapter 6 . 


\section{2}

\section{Experimental setup}

The random DFB fiber laser used in this experiment is similar to that presented in Figure 5.5, but with the addition of a phase modulator device between the SOA and the DS fiber. The experimental setup is shown in Figure 7.1(a). Two phase modulation devices were tested in the experiments: an electro-optic phase modulator (EOPM) and a fiber stretcher. Both devices modulate the optical phase, but the modulation proceeds in slightly different way in each case.

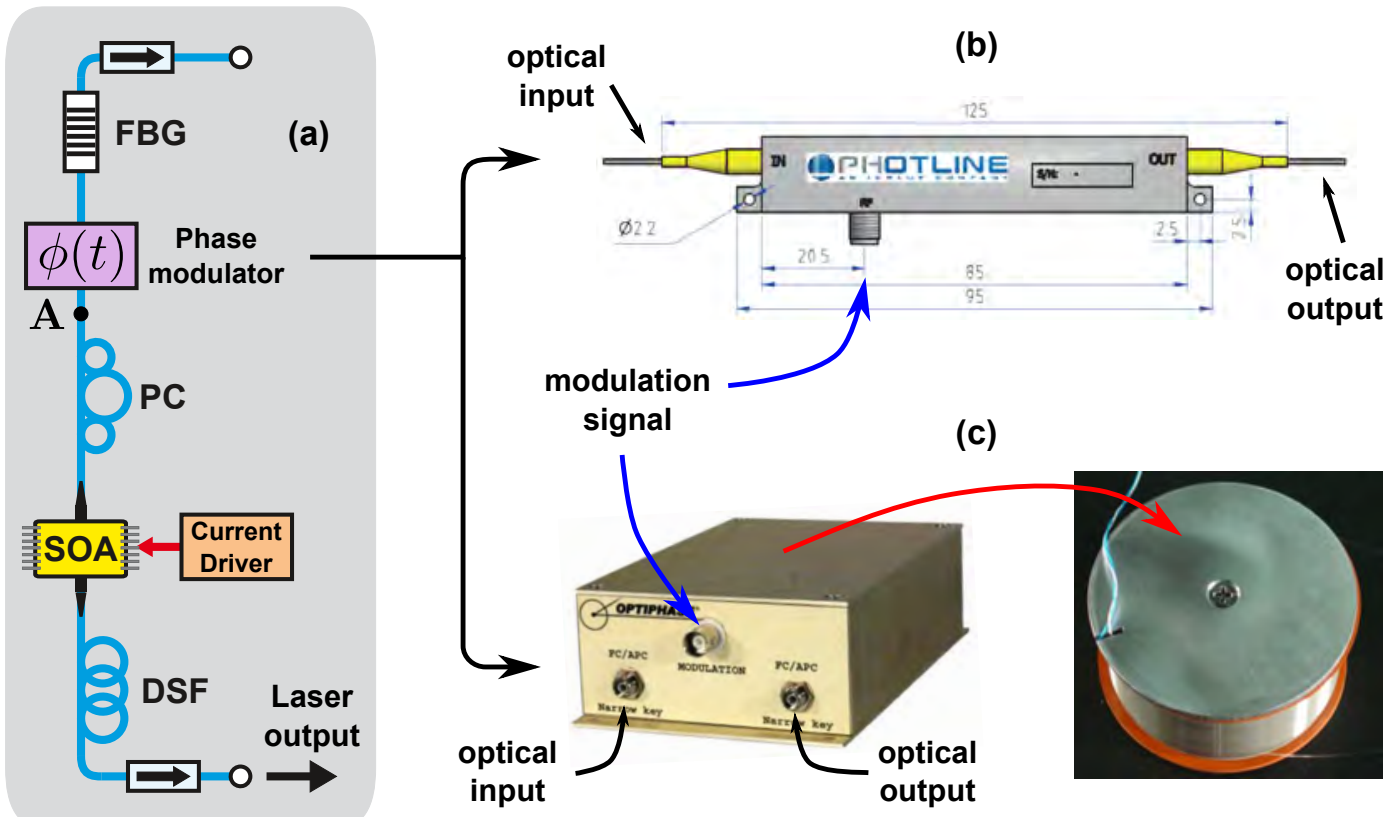

Figure 7.1: (a) Experimental setup of SOA-based random DFB fiber laser with intra-cavity phase modulation. (b) Electro-optic phase modulator. (c) Fiber stretcher phase modulator.

The EOPM used in the experiments was an iXblue MPZ-LN-40 (Figure 7.1(b)) [122], operating in the wavelength range 1530-1625 nm. It has an insertion loss of $\sim 2.5 \mathrm{~dB}$, not considering losses at the input/output connectors. Its electro-optical bandwidth is of $33 \mathrm{GHz}$. It is composed of Lithium Niobate (LiNb), which, under an applied voltage, modifies the optical path with a periodic transfer function with half-period $V_{\pi}$. Under low modulation frequencies $(\sim 50 \mathrm{kHz})$ the $V_{\pi}$ is $6 \mathrm{~V}$, and it increases up to $8.5 \mathrm{~V}$ under high modulation frequencies $(\sim 30 \mathrm{GHz})$. In this type of phase modulation, it is said that the phase is wrapped because the modulated phase is bounded in the range $[0, \pi]$. This limitation is eliminated when using fiber stretchers for phase modulation.

The fiber stretcher used, model Optiphase PZ2-SM2 (Figure 7.1(c)) [123], is a cylindrical piezoelectric transducer (PZT), over which a $40 \mathrm{~m}$-long fiber 
is coiled. By applying sinusoidal voltage waveforms to the PZT, the cylinder stretches and compresses, and the coiled optical fiber follows the modulation, hence the optical path is directly modulated. A drawback of this modulation format is the frequency response of the solid cylinder, which is highly nonlinear as shown in Figure 7.2(a). It has a resonance peak at $\sim 17 \mathrm{kHz}$, where the optical path displacement (OPD) is of $\sim 1000 \mu \mathrm{m} / \mathrm{V}$. The recommended operation bandwidth for the fiber stretcher device goes up to $10 \mathrm{kHz}$, which is shown in Figure 7.2(b). Different than EOPMs, fiber stretchers allow unwrapped phase modulation, and by using a sinusoidal modulation format of $6 \mathrm{Vpp} @ 1 \mathrm{kHz}$, the optical phase is modulated in the range $[0, \sim 42 \pi]$, about 42 times larger than that obtained with the available EOPM.

(a)
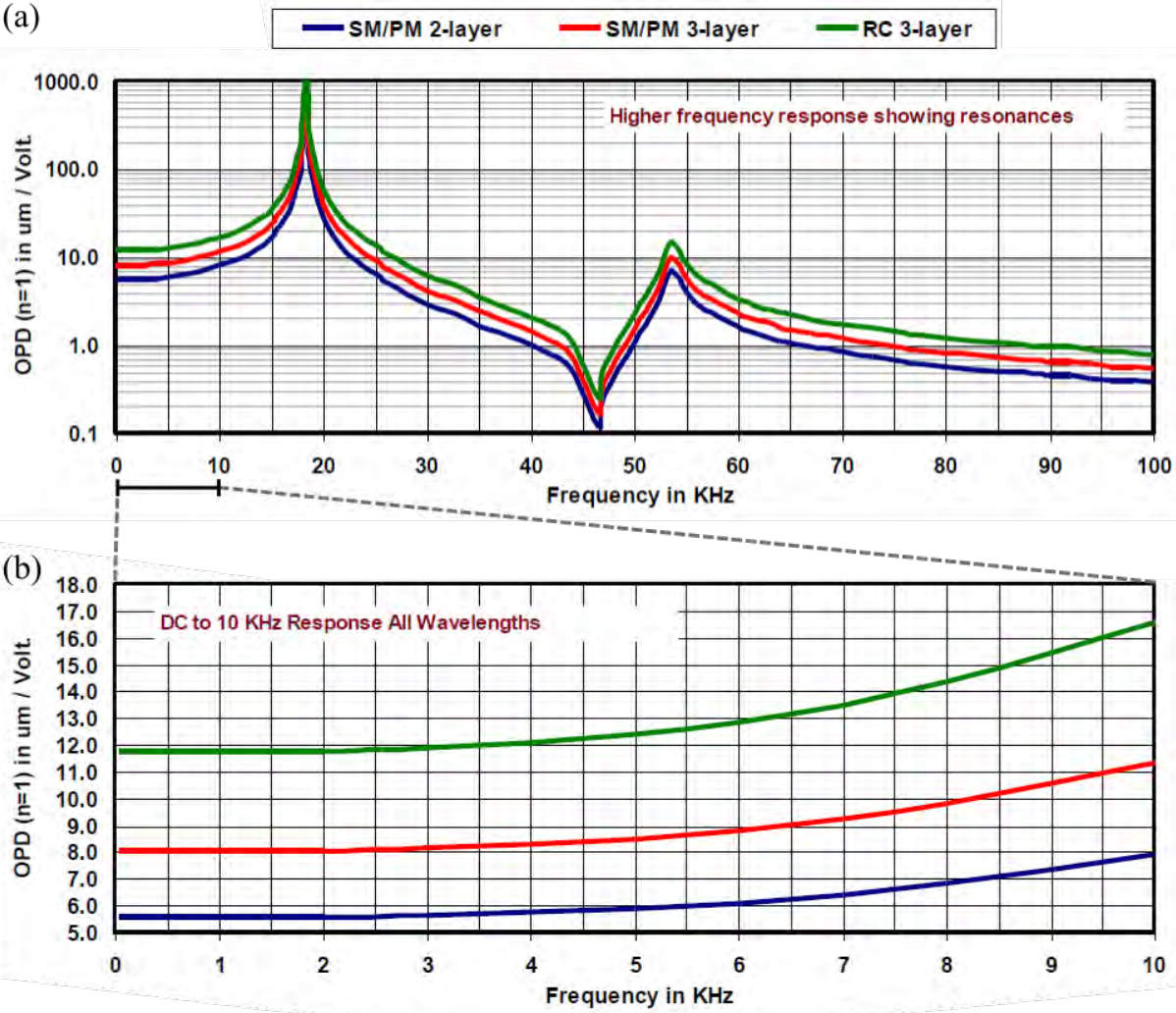

Figure 7.2: Frequency response of high-efficiency fiber stretcher Optiphase PZ2-SM2 [123]. SM2-layer (blue curve) corresponds to the model used in the experiments.

\section{3}

\section{Theoretical analysis}

We theoretically examine the experimental setup shown in Figure 7.1(a) by dividing it in two parts at point $\mathbf{A}$, such that each part is analyzed separately. At the bottom part, the analysis is the same as provided in chapter 5, with Rayleigh scattering providing randomly DFB, resulting in a 
intensity fluctuation highly frequency dependent (see Figure 5.1). The interest now is to evaluate the upper part, and then unite both parts in a complete theory.

For a theoretical analysis of the upper part (above point $\mathbf{A}$ in Figure 7.1), let us assume an ideal phase modulator that modulates the optical field periodically according to:

$$
\phi_{m}(t)=A_{m} \sin \left(2 \pi f_{m} t+\varphi_{m}\right)
$$

where $A_{m}, f_{m}$ and $\varphi_{m}$ are the amplitude, frequency and global phase of the modulation signal. In such way, a monochromatic optical field $E$, with amplitude $E_{0}$, angular frequency $\omega_{0}$ and wavenumber $k_{0}$ traversing the phase modulator would have its phase modulated by:

$$
\begin{aligned}
& E(z, t)=E_{0} \sin \left(\omega_{0} t+k_{0} z+\phi_{m}(t)\right) \\
& E(z, t)=E_{0} \sin \left(\omega_{0} t+k_{0} z+A_{m} \sin \left(2 \pi f_{m} t+\varphi_{m}\right)\right),
\end{aligned}
$$

where polarization dependence was neglected for simplicity.

In the particular configuration under analysis, a monochromatic wave entering the upper part of the cavity would experiment a dual-pass at the phase modulator, with a phase difference between the two passes defined by the length of fiber between the phase modulator and the FBG. Clearly, we are interested in analyzing monochromatic waves with frequencies lying within the FBG's reflectivity bandwidth, $\Delta \nu_{B}$, otherwise single-pass phase modulated signals would be transmitted through the FBG. Thus, when a monochromatic wave $E$, with optical frequency $\nu_{0}$ lying within $\Delta \nu_{B}$, inputs the upper part of the cavity at point $\mathbf{A}$, it would output at this same point but in the opposite direction with an optical field given by:

$$
\begin{aligned}
E(t)= & E_{0} \sin \left(\omega_{0} t+2 k_{0} r+\pi+\right. \\
& \left.A_{m}\left[\sin \left(2 \pi f_{m} t+\varphi_{m}\right)+\sin \left(2 \pi f_{m}(t-T)+\varphi_{m}\right)\right]\right),
\end{aligned}
$$

where $T=2 r(n / c)$, and $r$ is the length of optical fiber between the phase modulator and the FBG, which we measured to be $3 \mathrm{~m}$ in the experimental setup shown in Figure 7.1. The term $2 k_{0} r$ represents the phase propagation in a round-trip at the fiber of length $r$, and the $\pi$ deviation comes from the phase inversion at the FBG. The last two sine terms correspond to the double pass at the phase modulator, with one modulation delayed by $T$ from the other. The phase difference between the two phase modulations can be written as:

$$
\Delta \phi_{m}=2 \pi f_{m}(2 r n / c) .
$$


From Equation 7-5, it is observed that depending on the modulation frequency $f_{m}$, the dual-pass at the phase modulator can result in a phase modulation with twice the amplitude of a single pass (constructive interference), or a case of zero modulation, where the modulation from the first pass is completely canceled by the second pass (destructive interference). Of course, since $f_{m}$ can be continuously tuned, any case in between a constructive and a destructive inference from the two passes at the phase modulator is possible. An important outcome from Equation 7-5 is that phase modulation with low frequencies $\left(f_{m} \sim \mathrm{kHz}\right)$, gives a $\Delta \phi_{m}$ of approximately zero. Thus, the phase modulation is always amplified by a factor two for low modulation frequencies. For phase modulation with high frequencies $\left(f_{m} \sim 100 \mathrm{MHz}\right)$, a frequency increment of $16.67 \mathrm{MHz}$ changes the dual-pass phase modulation from cases with constructive interference to destructive interference. For example, a modulation frequency of $100 \mathrm{MHz}$ gives $\Delta \phi_{m}=6 \pi=0 \mathrm{rad}$ (constructive interference), while $f_{m}=116.67 \mathrm{MHz}$ results in $\Delta \phi_{m}=7 \pi=\pi \mathrm{rad}$ (destructive interference).

Hence, it is important to analyze cases with low $f_{m}$ and particularly high $f_{m}$ 's resulting in in-phase dual-pass phase modulation. Let us first analyze the case with low frequency modulation. As shown in section 5.4.3, the mode's linewidth $\Delta \nu$ of the SOA-based random DFB fiber laser is $\sim 4 \mathrm{kHz}$. Also, since it is a uni-dimensional laser with coherent feedback, satisfying the phase condition is required for laser action. In fact, as discussed in chapters 5 and 6 , laser pulses start being observed near the lasing threshold because of coherent reflectivity peaks. Thus, we expect that phase modulation with frequencies $f_{m}$ near the $\Delta \nu$ would result in the breaking of the phase condition, as the phase of each mode is shifted at every round-trip, preventing a constructive interference in multiple round-trips in the laser cavity.

Under high $f_{m}$ with in-phase dual-pass phase modulation, let say $f_{m}=100 \mathrm{MHz}$, a different scenario should be expected. By modulating the phase, multiple sidebands rise at the frequency spectrum, with a frequency shift from the carrier equal to an integer number of $f_{m}$. From an energy viewpoint, a single pass in any phase modulator corresponds to an energy transfer from the carrier to multiple sidebands. Thus, each reflectivity peak coming from the DSF in the bottom part of the experimental setup (below point $\mathbf{A}$ ) would experiment dual phase modulation (above point A) and transfer energy to its neighbors apart from an integer number of $f_{m}$. Modulating the phase with higher modulation amplitudes $A_{m}$ result in more energy being transferred from the carrier to sidebands. Thus, according to the simulation result provided in Figure 5.1, which showed that the maximum peak reflectivity is $11.3 \mathrm{~dB}$ above the mean value, by increasing the modulation amplitude $A_{m}$ 
from zero to higher values, the maximum peak reflectivity will transfer more energy to its neighbors than it will receive energy from them, simply because it is the maximum reflectivity. The energy transfer can be seen as an additional loss to the lasing mode, such that more gain would be required to compensate the losses and reach lasing threshold. It is useful to define an additional loss coefficient from phase modulation, which we define here as:

$$
\alpha_{\phi}(M)=\frac{g\left(i_{T H}\left(A_{m}=0\right)\right)}{g\left(i_{T H}\left(A_{m}=M\right)\right)},
$$

where $g(i)$ represents the gain provided by the SOA at a driven current $i$, and $i_{T H}$ is the threshold current for laser action. Note that the numerator in Equation 7-6 corresponds to the gain promoted by the SOA at the laser threshold without phase modulation, while the denominator is the gain required to reach the threshold for any given $A_{m}$ with arbitrary amplitude $M$. When $M=0$, the numerator is equal to the denominator, such that the added loss coefficient equals zero. Increasing the modulation amplitude, more gain should be required to compensate the added losses and reach laser action, such that $\alpha_{\phi}$ results in a value lower than one - higher threshold current. In this way, laser action is expected to be prevented by the breaking of the gain condition, even under phase-modulation.

To calculate the amount of energy loss due to phase modulation, we return to the theoretical case with a monochromatic wave traversing the phase modulator twice. The result is similar to the Bessel function of the first kind of order zero, but since the phase modulation is twice as large, the carrier decreases more sharply and the sidebands increase more rapidly. The theoretical result for carrier decay and sidebands increase in a dual phase modulation scheme is shown in Figure 7.3. To calculate the theoretical added loss coefficient, we should not consider a monochromatic case, but rather an optical wave with intensity distribution given by the reflectivity profile of Rayleigh backscattering in the optical fiber. The intensity calculation at a frequency $\nu$ is given as follows:

$$
\begin{aligned}
I(\nu)= & w_{c}\left(A_{m}\right) \cdot I(\nu)+ \\
& w_{1 s t}\left(A_{m}\right) \cdot\left[I\left(\nu-f_{m}\right)+I\left(\nu+f_{m}\right)\right]+ \\
& w_{2 n d}\left(A_{m}\right) \cdot\left[I\left(\nu-2 f_{m}\right)+I\left(\nu+2 f_{m}\right)\right]+ \\
& w_{3 r d}\left(A_{m}\right) \cdot\left[I\left(\nu-3 f_{m}\right)+I\left(\nu+3 f_{m}\right)\right]+\ldots
\end{aligned}
$$

where $I(\nu)$ is the intensity at frequency $\nu$, and $w$ is a weighting factor that depends on the modulation amplitude $A_{m}$. The weights as precisely the curves shown in Figure 7.3 for the monochromatic case. For $A_{m}=0$, the carrier's weight 
$w_{c}$ equals 1 , and all other weights equal zero. When $A_{m}$ increases, the weight of the carrier decreases while the weights of the first $\left(w_{1 s t}\right)$, second $\left(w_{2 n d}\right)$, third $\left(w_{3 r d}\right), \ldots$ sidebands increase. Note that, before reaching laser action, the phases at each optical frequency throughout the FBG's bandwidth are random since it is ASE light that initiates laser action, thus the intensity calculation should be done incoherently, as per Equation 7-7. By assuming that the laser threshold is reached at $A_{m}=0$, we can calculate the additional loss coefficient by calculating the decrease in the maximum peak reflectivity when $A_{m}$ increases. The simulation result is shown in the black curve in Figure 7.3, where we considered a simulation bandwidth of $5 \mathrm{GHz}$ in the calculation.

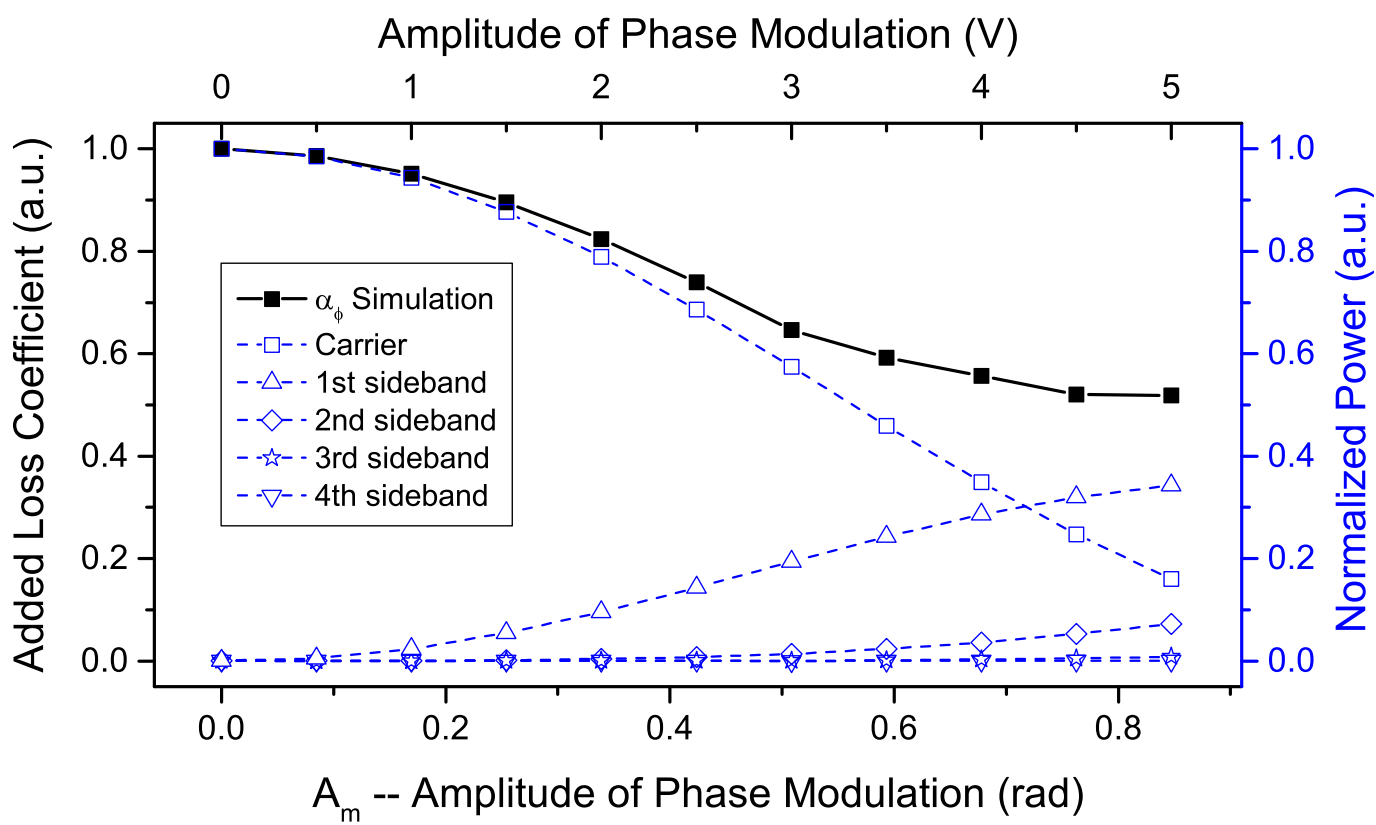

Figure 7.3: Theoretical result for power distribution between a monochromatic carrier and its sidebands after dual phase modulation with in-phase contributions (blue curves). The black curve shows a simulation result for the added loss coefficient for the random DFB fiber laser when operating with in intra-cavity phase modulation with modulation amplitude $A_{m}$.

According to Figure 7.3, the added loss coefficient is expected to follow the carrier losses of a monochromatic wave for low modulation amplitudes, which is reasonable since only a small portion of energy is being transferred from the maximum reflectivity peak to neighbors for low $A_{m}$. Interestingly, increasing $A_{m}$ above $0.3 \mathrm{rad}$, the added loss coefficient detaches from the carrier loss curve. This means that either one of two phenomena might be happening. 1) The maximum peak intensity at $\nu_{\max }$ still lies in the same optical frequency after phase modulation, but at the same time that it loses energy to neighboring frequencies apart from an integer number of $f_{m}$, these neighboring frequencies also lose energy to $\nu_{\max }$, such that the effective loss at 
the maximum intensity peak is alleviated by the presence of neighboring peaks. 2) The Rayleigh intensity distribution across the frequency bandwidth under analysis is re-balanced under phase modulation, so that enough energy can be transferred to a certain frequency that ends up overcoming the maximum reflectivity peak formerly at $\nu_{\max }$, and a new maximum value is achieved at a different frequency. This behavior becomes more evident for $A_{m}$ higher than $0.5 \mathrm{rad}$, where the weight of the first sidebands start being relevant, and the added loss coefficient reaches a nearly stable value for $A_{m}>0.65 \mathrm{rad}$.

\section{4}

\section{Results and discussions}

The experiments developed for analysis of intra-cavity phase-modulation considered the impacts of two modulation parameters: amplitude, $A_{m}$, and frequency, $f_{m}$. As expected, we observed that phase-modulation with $f_{m}$ comparable to the mode's linewidth $\Delta \nu$ is a highly efficient way to extinguish laser action even for high pumping. For instance, when driving the SOA with a current of $400 \mathrm{~mA}$ in the absence of phase-modulation, the random DFB fiber laser operates in a CW-like regime, similar to the result shown in Figure 5.7(d). Using either the EOPM or the fiber stretcher as phase modulator, and varying the optical phase in the range $[0,2 \pi]$ with a frequency of $1 \mathrm{kHz}$, the $\mathrm{CW}$-like behavior ceases completely. Phase variations with frequencies $\sim \Delta \nu$ hinder phase-preservation over a round-trip in the half-open cavity, such that the phase condition for laser action is broken (see Eq. 5-4). This result is also a proof that the one-dimension random DFB fiber laser is indeed coherent, with field interference playing a key role in laser action.

We noticed to be a minimum value of phase variation rate, i.e., $d \phi / d t$, necessary to break the phase condition. If a constant frequency is set for phase modulation, then $A_{m}$ defines the minimum $d \phi / d t$. Since the EOPM phasevariation coefficient is $\sim 0.52 \mathrm{rad} / \mathrm{V}$, and the fiber stretcher's is $\sim 22.3 \mathrm{rad} / \mathrm{V}$, the fiber stretcher device is able to break the phase condition for much smaller applied voltages. In addition, as it sustains unwrapped phase modulation, it could still cease lasing action by using high modulation amplitudes even for very small modulation frequencies, in the order of tens Hz. For such small modulation frequencies, a slow phase variation in the range of $[0, \pi]$ was not enough to break the phase condition, and the EOPM failed to prevent laser action. In this context, the advantage of using EOPMs comes when high modulation frequencies are employed, as fiber stretchers are not suited for high frequency modulation.

For phase-modulation frequencies far above $\Delta \nu$, two cases were analyzed: 


$$
\begin{aligned}
& \text { 1) } \Delta \nu \ll f_{m} \ll \Delta \nu_{B} \\
& \text { 2) } \Delta \nu \ll \Delta \nu_{B}<f_{m},
\end{aligned}
$$

where we employed the same FBG used in the experiments described in the previous chapters, with $\Delta \nu_{B}=7.5 \mathrm{GHz}$. To experimentally analyze the first case, we used the EOPM as phase modulator and set $f_{m}$ to $100 \mathrm{MHz}$. The laser output power was measured as a function of SOA driving current. We first measured a reference power-curve, which was obtained in the absence of phase-modulation, and then measured multiple power-curves for $A_{m}$ up to 5 Vpp. Measurement results are shown in Figure 7.4. It is first noted that the generation threshold obtained for the reference power-curve is higher than that shown in Figure 5.6. This difference is attributed to the insertion loss of the EOPM, which adds a $\sim 2.5 \mathrm{~dB}$ loss to the cavity, thus the system demands additional gain to start lasing. A more significant aspect observed is the fact that laser emission is present for all cases, even for high $A_{m}$, which showed a higher generation threshold. Note that, for the high modulation frequency used, $100 \mathrm{MHz}$, and also high amplitude modulations (5 Vpp), the phase variation rate is large, but lasing action is still observed.

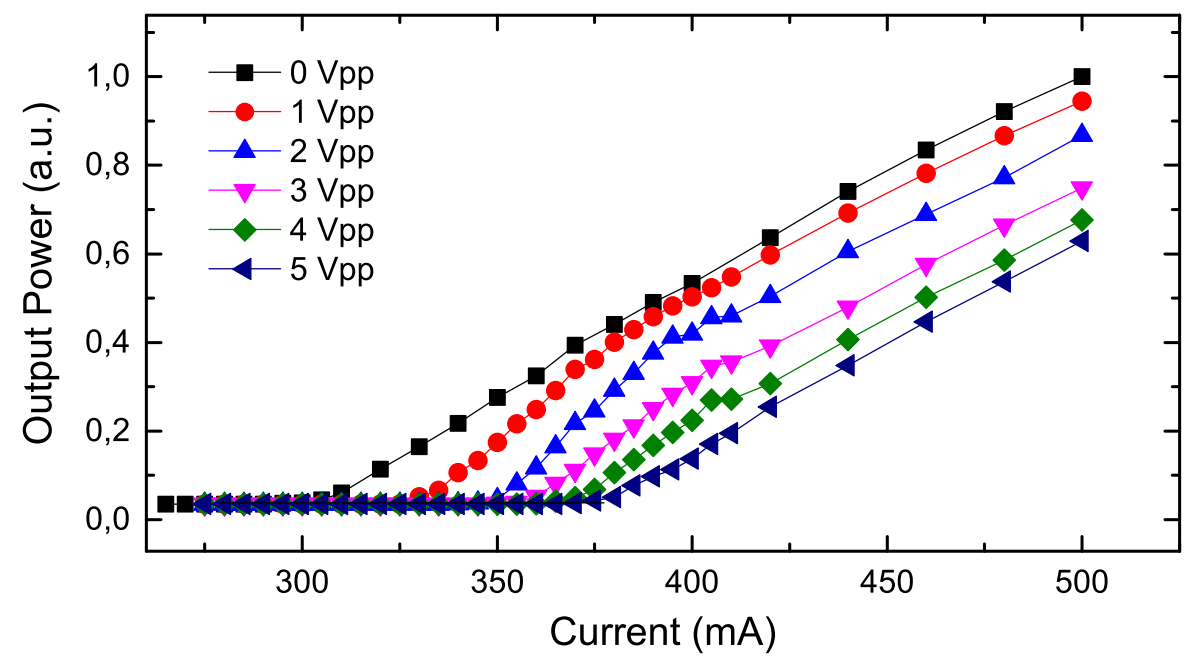

Figure 7.4: Measurement of random DFB fiber laser output power increase with SOA driving current for different phase-modulation voltages, at a modulation frequency of $100 \mathrm{MHz}$.

The experimental result shown in Figure 7.4 agrees with theoretical predictions. By applying an intra-cavity phase modulation signal, additional losses are imposed to the cavity, and more gain is required to reach the lasing threshold, which occurs at higher SOA driving currents when $A_{m}$ increases. Before calculating the experimental added loss coefficient and compare with the simulation result, we must calibrate $A_{m}$ with real voltage amplitudes set to the EOPM. 
We conducted the experiment shown in Figure 7.5(a), to characterize the energy distribution among an optical carrier and its sidebands when traversing the phase modulator twice. The experiment is given as follows. Light from a $3 \mathrm{kHz}$-linewidth laser is directed to the EOPM by means of an optical circulator. The phase-modulated light impinges on the FBG and is reflected back to the phase modulator, which again modulates the incoming light. The dual phase-modulated light spectrum is measured in a high-resolution OSA (resolution of $20 \mathrm{MHz}$ ).

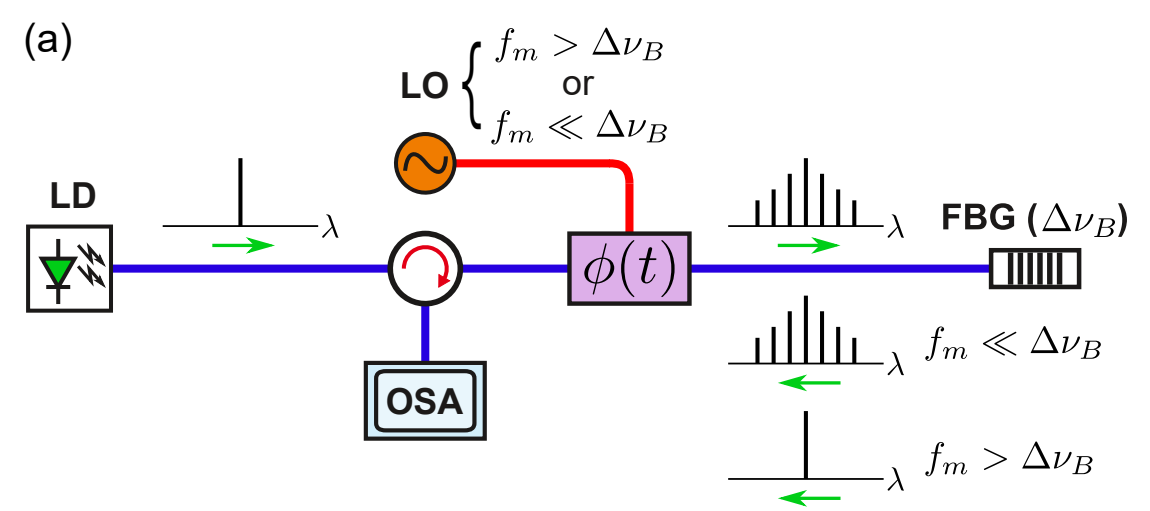

(b)

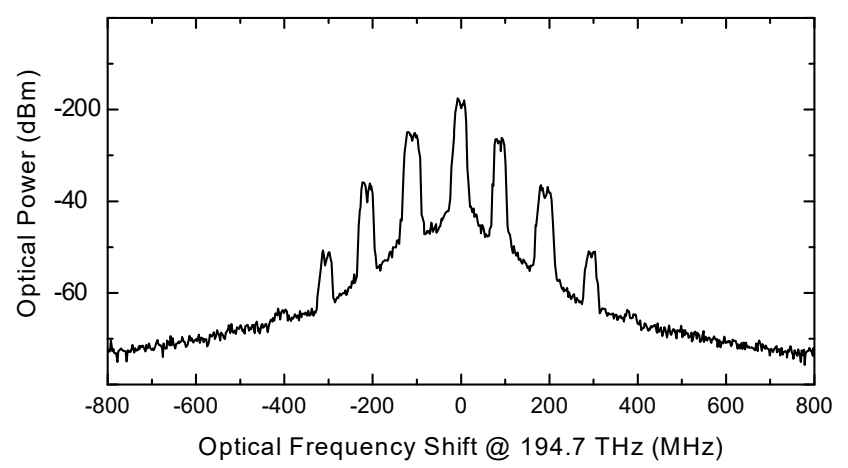

Figure 7.5: Characterization of experimental set: double pass at EOPM + FBG. (a) Experimental configuration. (b) Example of spectral measurement at OSA using $f_{m}=100 \mathrm{MHz}$.

As mentioned in the previous section, the modulation frequency plays an important role in the experiment. If $f_{m} \ll \Delta \nu_{B}$, i.e. case 1) defined in 7-8, then all sidebands are reflected at the FBG. When the reflected phase-modulated signal traverses the phase modulation, then the carrier and sidebands are individually phase modulated, all exchanging energy between each other. A measured optical spectrum for this case is shown in Figure 7.5(b). On the other hand, for modulation frequencies higher than the FBG bandwidth, i.e., case 2) of 7-8, then the sidebands are transmitted through the FBG, and the carrier experiments an even more severe attenuation, as sidebands are not present to transfer back part of their energy to the carrier in the second pass in the phase modulator. 
By fitting experimental data to simulation results, we found a factor of $\sim 0.17$ linking $A_{m}$, in radians, to applied modulation amplitude, in volts. Then, by making a correspondence between SOA-current in optical gain with the usage of Figure 2.15, we calculated the experimental added loss coefficient and plot it as a function of $A_{m}$. The result is shown in Figure 7.6, where the simulation results from the previous section are also displayed for comparison. As predicted, the experimental $\alpha_{\phi}$ follows the carrier power up to $A_{m} \sim 0.3 \mathrm{rad}$, when it detaches and exhibits higher values than the carrier loss. Both experimental and simulation curves of $\alpha_{\phi}$ detaches from the carrier curve and exhibit higher values (lower added loss), reaching a nearly stable value for $A_{m} \sim 0.65 \mathrm{rad}$, just as expected from simulation predictions.

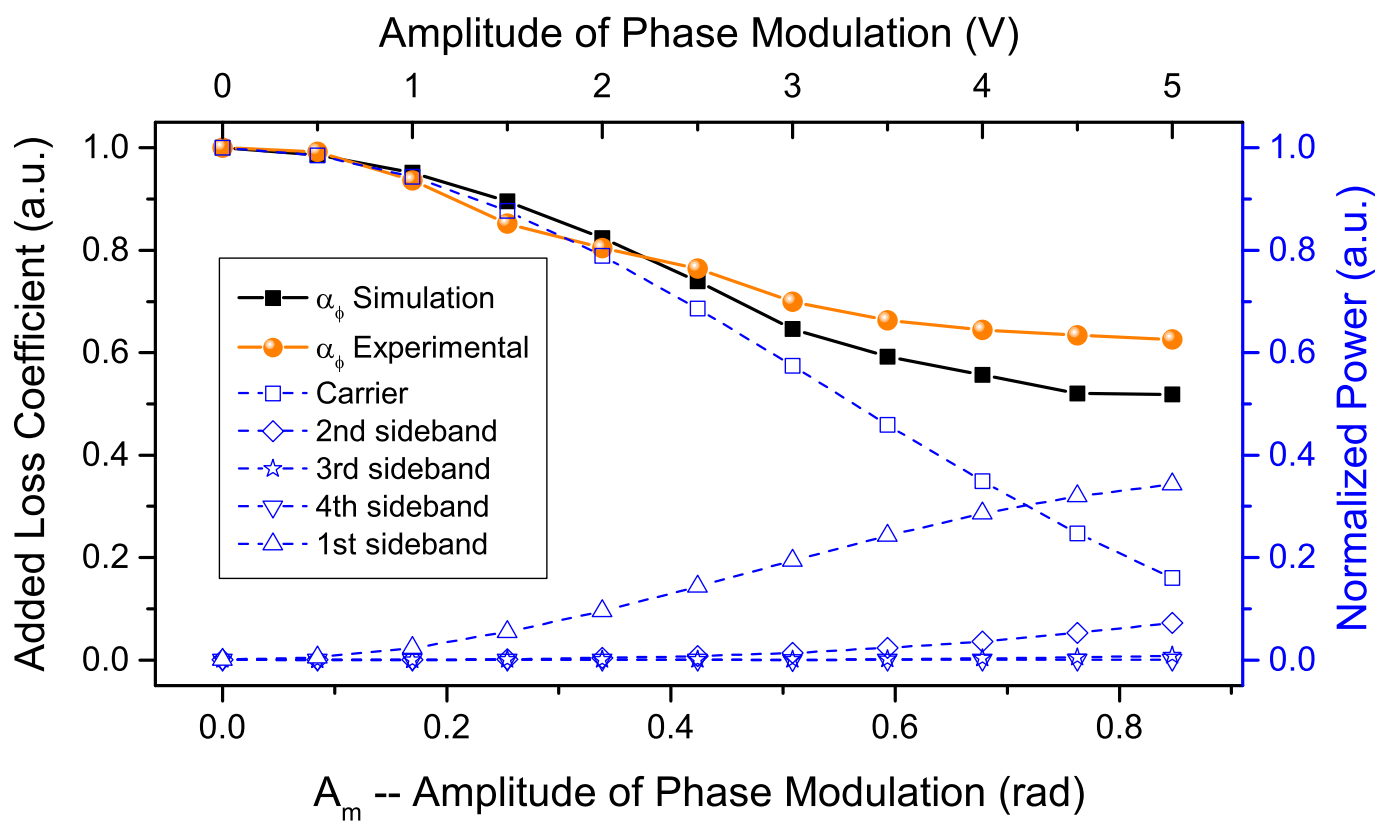

Figure 7.6: Comparison between experimental and simulation results for the added loss coefficient. Blue curves show the energy distribution of a theoretical monochromatic wave and its sidebands when under dual phase-modulation.

The similarity between simulation added loss and experimental added loss coefficients shows that the theoretical model developed for Rayleigh scattering intensity fluctuations can also be used to predict lasing action under intra-cavity phase modulation conditions. For high modulation amplitudes, a small difference between simulation and experimental results is still observed though. This difference is probably due to the narrower frequency bandwidth $\Delta \nu_{B}$ used in the simulation, which was of $5 \mathrm{GHz}$, while the actual $\Delta \nu_{B}$ is of $7.5 \mathrm{GHz}$. As shown in Figure 3.6(a), wider bandwidths results in higher probabilities of having a high peak reflectivity starting laser action. Similarly, in the case with intra-cavity phase modulation, wider bandwidths provide more possibilities of intensity peaks, with energy re-balanced from dual phase 
modulation, able to start laser action, and an increase in the added loss coefficient (lower cavity loss) should be observed when wider bandwidths are considered.

An important conclusion to be taken from the experiments conducted in this section is that the breaking of phase-condition is more critical for laser action than of gain-condition. The additional losses imposed to the cavity when applying high frequency phase-modulation can be compensated if enough gain is provided. However, breaking the phase condition showed to completely cease laser action, which was observed when low frequency $(<\Delta \nu)$ phase-modulation was applied to the cavity. 


\section{8 \\ Summary and future works}

\section{1 \\ Summary}

This thesis analyzed the mode dynamics of random DFB fiber lasers, focusing on the key role of Rayleigh scattering in the random lasing process. We developed a theoretical model for prediction of intensity fluctuations of Rayleigh backscattering in optical fibers. The model was built based on two key elements that explain intensity fading of Rayleigh backscattering of coherent light: sub-micron and centimetric refractive index fluctuations, the former related to density fluctuations of amorphous fibers, and the latter to residual stress resulting from the manufacturing process. Different than many models that assume a random scattering amplitude, it was shown that intensity fluctuations exist even for constant scattering amplitudes. Intensity fluctuations were evaluated in the frequency domain, and it was shown that backscattered intensity fluctuates more for longer fibers. The model was used to simulate $\Phi$-OTDR measurements clearly showing how external fluctuations affect local properties of the fiber and how it can be used to predict or optimize experimental parameters to improve $\Phi$-OTDR measurements.

An experiment was performed to measure intensity fluctuations of Rayleigh backscattering in time domain. To compare the experimental result with simulation results, the model was modified to account for the source linewidth. The remarkable coincidence between the statistics of experimental fluctuations with those obtained from the theoretical model in the frequency domain strongly supports the statement that Rayleigh backscattering is an ergodic process exhibiting ergodicity in the time-frequency sense, a relevant result for many research areas.

It should be noted that the model presented was developed focusing on guided light in optical fibers, but the basic assumptions are valid for any scattering medium. Thus, if the $1 / \mathrm{r}^{2}$ factor is included in Equation 3-5 instead of the exponential attenuation factor of the guided light, scintillation and Lidar fluctuations of coherent backscattered light in the atmosphere are straightforwardly modeled, the random fluctuations of the refractive index now 
being generated by wind or turbulence fluctuations of the atmospheric density.

Mode characteristics of random DFB fiber lasers with SOA gain were presented. The model predicting Rayleigh backscattering intensity fluctuations was employed to explain mode structure and dynamics. Simulation results indicate that the nonuniform longitudinal mode spacing is due to the random variations in the refractive index caused by residual stress. Small fluctuations of the environmental conditions give rise to a scintillation effect in the Rayleigh backscattered light, therefore modulating the effective fiber reflectivity. The model also predicts that the scintillation effect induces randomly driven passive Q-switched pulses near lasing threshold. The statistical prediction of the model for the threshold current remarkably agrees with the experimental results. Experimental results confirms that single longitudinal mode operation is only possible close to the threshold current, whereas multimode operation dominates when the SOA current is increased. Mode beating in multimode regime was observed to continuously cover the full laser emission band. Mode linewidth is $\leq 4 \mathrm{kHz}$ and it increases up to $7 \mathrm{kHz}$ at higher driving currents, an effect of mode competition, which reduces the average lifetime of cavity modes. Spectral analysis indicates that multimode operation dominates the laser output at high currents, when mode output power is limited by mode competition.

To verify if the model predictions extend to RFL with distributed gain, a Brillouin-based RFL was experimentally analyzed. Indeed, we have shown that narrow linewidth single-mode operation is only possible when pumping near the generation threshold and in a pulsed emission format. Pulses duration is compatible with the fiber's phase-fluctuation rate and governed by Rayleigh scattering. Above threshold the presence of strong beat notes observed in the laser's output indicates that multiple modes are satisfying the lasing conditions simultaneously. At high pumping the laser operation is quasi-CW, exhibiting low frequency fluctuations governed by Rayleigh scattering and high frequency fluctuations given by multimode beating. Although the selfheterodyne measurement technique is misleading and could lead to the wrong conclusion of single longitudinal mode operation, it actually measures the spectral linewidth of each lasing mode, even in multimode operation. It is important to note that Rayleigh backscattering properties govern the mode behavior of Brillouin-based RFLs and do not depend on the particular laser cavity configuration studied in this work. Our results provide experimental proof that Brillouin RFLs operate in a continuous multimode regime when pumped above the threshold, with a laser linewidth determined by the Brillouin gain spectral width. 
Last, intra-cavity phase modulation was experimentally analyzed. We showed that low frequency phase-modulation is an efficient way to control random laser action, which is ceased by breaking the phase condition. As already expected due to uni-dimensional configuration with linear half-open cavity, the breaking of the phase condition proofs that the laser is indeed coherent, with field interference being required for lasing. Interestingly, by modulating the cavity's phase with high frequency, the laser action may also cease, but by breaking the gain condition.

\section{2}

\section{Future works}

Random DFB fiber lasers are a recent field in laser physics, which is still far from being fully understood. We hope this work encourage and help others to continue the study of such interesting field, which still has a lot of challenges to be solved. Possible directions for future works are listed below.

- The Rayleigh scattering model developed in this thesis has been studied in the light of random DFB fiber lasers, but $\Phi$-OTDR applications have also been discussed. Experimental measurements for $\Phi$-OTDR could be tested and compared with model's prediction. As mentioned in chapter 3, the laser's linewidth is included in the model, and $\Phi$-OTDR schemes with sources of multiple linewidths could be tested for ultimate validation.

- Theoretical results indicated that residual stress in optical fibers resulting from the manufacturing process is one of the most fundamental aspects explaining Rayleigh scattering intensity fluctuations. Although very small index fluctuations still resulted in significant intensity fluctuations of Rayleigh scattering as shown in Figure 3.7, a manufacturing process without loop control acting on the fiber residual stress might bring new insights to full comprehension of the phenomenon.

- Another interesting aspect to be explored is the study of mode dynamics of random fiber lasers in full-open cavity configuration. Although mentioned in [10] that a linear half-open cavity with feedback defined by a mirror at one end and distributed Rayleigh scattering at the the other end is equivalent to a a full-open cavity, with Rayleigh scattering promoting feedback at both ends, the mode structure might be a bit different in each case. In a half-open cavity, the mirror promoting fixed 
feedback can be seen as a second fiber which has a reflectivity profile that is the exact image of the real-fiber. Thus, the phase-variations due to environmental changes along the real-fiber remain the same in the image-fiber. In a full open cavity, since the phase variations along two fibers proceed in different ways, there might be a difference in mode dynamics. However, in terms of power reflectivity, the fixed mirror provides much greater feedback ( $80 \%$ or higher), while Rayleigh feedback mean power reflectivity is extremely low $(<0.01 \%)$. Hence, SOA might not be the best gain option to study such cases given the small gain provided by such devices. Still, further analysis of mode lifetime and pulses duration could be an interesting direction for future study.

- We showed in chapter 3 that Rayleigh scattering exhibits an exponential probability distribution in both temporal domain (for a narrow linewidth light), and optical frequency domain (for a short time interval). Due to the lack of appropriate equipment, experimental measurements could not be performed in the optical frequency domain for short time intervals, as the sweeping wavelength OSAs available take long time for each measurement. However, with the possession of a spectrometer, which measures light intensity throughout an optical frequency bandwidth at the same instant, one could easily validate our theoretical model experimentally in the optical frequency domain. 


\section{Bibliography}

[1] S. K. Turitsyn, S. A. Babin, A. E. El-Taher, A. E. El-Taher, P. Harper, D. V. Churkin, S. I. Kablukov, J. D. Ania-Castañón, V. Karalekas, and E. V. Podivilov, "Random distributed feedback fibre laser," Nature Photonics, vol. 4, p. 231-235, 2010.

[2] G. P. Agrawal, Fiber-Optic Communications Systems, ser. Wiley Series in Microwave and Optical Engineering. John Wiley and Sons, 2002.

[3] T. W. Kohlgraf-Owens and A. Dogariu, "Transmission matrices of random media: means for spectral polarimetric measurements," Opt. Lett., vol. 35, no. 13, pp. 2236-2238, Jul 2010.

[4] B. Redding, S. F. Liew, R. Sarma, and H. Cao, "Compact spectrometer based on a disordered photonic chip," Nature Photonics, vol. 7, no. 9, pp. 746-751, Sep 2013.

[5] D. S. Wiersma, M. P. van Albada, and A. Lagendijk, "Random laser?" Nature, vol. 373, no. 6511, pp. 203-204, Jan 1995.

[6] R. Ambartsumyan, N. Basov, P. Kryukov, and V. Letokhov, "A laser with a nonresonant feedback," IEEE Journal of Quantum Electronics, vol. 2, no. 9, pp. 442-446, Sep. 1966.

[7] N. M. Lawandy, R. M. Balachandran, A. S. L. Gomes, and E. Sauvain, "Laser action in strongly scattering media," Nature, vol. 368, no. 6470, pp. 436-438, Mar 1994. [Online]. Available: https://doi.org/10.1038/368436a0

[8] D. S. Wiersma, "The physics and applications of random lasers," Nature Physics, vol. 4, no. 5, pp. 359-367, May 2008.

[9] C. J. S. de Matos, L. de S. Menezes, A. M. Brito-Silva, M. A. Martinez Gámez, A. S. L. Gomes, and C. B. de Araújo, "Random fiber laser," Phys. Rev. Lett., vol. 99, p. 153903, Oct 2007.

[10] S. K. Turitsyn, S. A. Babin, D. V. Churkin, I. D. Vatnik, M. Nikulin, and E. V. Podivilov, "Random distributed feedback fibre lasers," Physics Reports, vol. 542, no. 2, pp. 133-193, 2014. 
[11] Z. Wang, H. Wu, M. Fan, Y. Rao, I. Vatnik, E. Podivilov, S. Babin, D. Churkin, H. Zhang, P. Zhou, H. Xiao, and X. Wang, "Random fiber laser: simpler and brighter," Opt. Photon. News, vol. 25, p. 30, 2014.

[12] P. Tovar, G. Temporão, and J. P. von der Weid, "Longitudinal mode dynamics in soa-based random feedback fiber lasers," Opt. Express, vol. 27, no. 21 , pp. $31001-31012$, Oct 2019.

[13] P. Tovar, Y. H. Luis, G. Temporão, and J. P. von der Weid, "Longitudinal modes in random feedback fiber lasers," in 2019 Conference on Lasers and Electro-Optics (CLEO), 2019, pp. 1-2.

[14] S. A. Babin, A. E. El-Taher, P. Harper, E. V. Podivilov, and S. K. Turitsyn, “Tunable random fiber laser," Phys. Rev. A, vol. 84, p. 021805, Aug 2011.

[15] S. A. Babin, "New schemes of raman fiber lasers with random distributed feedback," in CLEO Pacific Rim Conference 2018. Optical Society of America, 2018, p. Th2A.1.

[16] L. Zhang, Y. Wang, Y. Xu, S. Gao, D. Zhou, L. Chen, and X. Bao, "Coherent brillouin random fiber laser for application in phase-sensitive optical time domain reflectometry," in 2018 Conference on Lasers and Electro-Optics (CLEO), 2018, pp. 1-2.

[17] L. Zhang, Y. Xu, S. Gao, B. Saxena, L. Chen, and X. Bao, "Multi-wavelength coherent brillouin random fiber laser with high optical signal-to-noise ratio," in Conference on Lasers and Electro-Optics. Optical Society of America, 2017, p. SM2L.6. [Online]. Available: http://www.osapublishing.org/abstract.cfm?URI=CLEO_SI-2017-SM2L.6

[18] M. Pang, X. Bao, L. Chen, Z. Qin, Y. Lu, and P. Lu, "Frequency stabilized coherent brillouin random fiber laser: theory and experiments," Opt. Express, vol. 21, no. 22, pp. 27 155-27 168, Nov 2013. [Online]. Available: http://www.opticsexpress.org/abstract.cfm?URI=oe-21-22-27155

[19] Z. Zhou, L. Chen, and X. Bao, "High efficiency brillouin random fiber laser with replica symmetry breaking enabled by random fiber grating," Opt. Express, vol. 29, no. 5, pp. 6532-6541, Mar 2021. [Online]. Available: http://www.opticsexpress.org/abstract.cfm?URI=oe-29-5-6532

[20] G. Yin, B. Saxena, and X. Bao, "Tunable er-doped fiber ring laser with single longitudinal mode operation based on rayleigh backscattering in single mode fiber," Opt. Express, 
vol. 19, no. 27, pp. 25981-25989, Dec 2011. [Online]. Available: http://www.opticsexpress.org/abstract.cfm?URI=oe-19-27-25981

[21] T. Zhu, X. Bao, and L. Chen, "A single longitudinal-mode tunable fiber ring laser based on stimulated rayleigh scattering in a nonuniform optical fiber," J. Lightwave Technol., vol. 29, no. 12, pp. 1802-1807, Jun 2011. [Online]. Available: http://jlt.osa.org/abstract.cfm?URI=jlt-29-12-1802

[22] H. A. Shawki, H. E. Kotb, and D. Khalil, "Narrow line width dual wavelength edfa based random fiber laser," in 2018 35th National Radio Science Conference (NRSC), 2018, pp. 433-438.

[23] A. A. Fotiadi and R. V. Kiyan, "Cooperative stimulated brillouin and rayleigh backscattering process in optical fiber," Opt. Lett., vol. 23, no. 23, pp. 1805-1807, Dec 1998. [Online]. Available: http://ol.osa.org/abstract.cfm?URI=ol-23-23-1805

[24] M. Nakazawa, "Rayleigh backscattering theory for single-mode optical fibers," J. Opt. Soc. Am., vol. 73, no. 9, pp. 1175-1180, Sep 1983.

[25] P. Healey, "Fading in heterodyne OTDR," Electronics Letters, vol. 20, pp. 30-32(2), January 1984.

[26] — - "Fading rates in coherent OTDR," Electronics Letters, vol. 20, pp. 443-444(1), May 1984.

[27] — - "Statistics of Rayleigh backscatter from a single-mode optical fibre," Electronics Letters, vol. 21, pp. 226-228(2), March 1985.

[28] J. Zhou, Z. Pan, Q. Ye, H. Cai, R. Qu, and Z. Fang, "Characteristics and explanations of interference fading of a $\phi$-otdr with a multi-frequency source," Journal of Lightwave Technology, vol. 31, no. 17, pp. 2947-2954, Sep. 2013.

[29] L. B. Liokumovich, N. A. Ushakov, O. I. Kotov, M. A. Bisyarin, and A. H. Hartog, "Fundamentals of optical fiber sensing schemes based on coherent optical time domain reflectometry: Signal model under static fiber conditions," Journal of Lightwave Technology, vol. 33, no. 17, pp. 36603671, Sep. 2015.

[30] C. D. Poole, "Measurement of polarization-mode dispersion in singlemode fibers with random mode coupling: errata," Opt. Lett., vol. 14, no. 15, pp. 829-829, Aug 1989. [Online]. Available: http://ol.osa.org/abstract.cfm?URI=ol-14-15-829 
[31] A. L. Schawlow and C. H. Townes, "Infrared and optical masers," Phys. Rev., vol. 112, pp. 1940-1949, Dec 1958.

[32] D. Strickland and G. Mourou, "Compression of amplified chirped optical pulses," Optics Communications, vol. 56, no. 3, pp. 219-221, 1985.

[33] B. E. A. Saleh and M. C. Teich, Fundamentals of Photonics, 2nd ed. John Wiley and Sons, 2007.

[34] K. Peterman and G. Arnold, "Noise and distortion characteristics of semiconductor lasers in optical fiber communication systems," IEEE Journal of Quantum Electronics, vol. 18, no. 4, pp. 543-555, April 1982.

[35] B. G. Streetman and S. K. Banerjee, Solid State Electronic Devices, 6th ed. Pearson Education Inc., 2006.

[36] S. M. Sze and K. K. Ng, Physics of Semiconductor Devices, 3rd ed. John Wiley and Sons, 2007.

[37] M. J. Connelly, Semiconductor Optical Amplifiers. Kluwer Academic Publishers, 2004.

[38] SOA1013SXS - High-Speed Optical Shutter/Switch, Thorlabs Inc., https://www.thorlabs.com/newgrouppage9.cfm?objectgroup_id=4336\& $\mathrm{pn}=\mathrm{SOA} 1013 \mathrm{~S} X \mathrm{~S}$.

[39] M. M. Resende, P. Tovar, G. C. Amaral, and J. P. von der Weid, "Overcoming the maximum amplification limit of coherent optical pulses in semiconductor optical amplifiers with time-polarization multiplexing," Optical Engineering, vol. 56, no. 11, pp. $1-3,2017$.

[40] F. Mayinger and O. Feldmann, Optical Measurements, 2nd ed. Springer, 2001.

[41] H. Jiang, Q. He, Z. Wang, L. Tang, and X. Shang, "Continuous measurement of distributed vibration using coherent rayleigh backscattering," in 2019 International Conference on Electronic Engineering and Informatics (EEI), Nov 2019, pp. 49-52.

[42] W. Yan-Hong, N. Guo-Qiang, G. Pan, and G. Kun, "Theoretical analysis on coherent noise by rayleigh backscattering in bidirectional transmission system with single mode fiber," in 2009 International Forum on Information Technology and Applications, vol. 1, May 2009, pp. 209-212. 
[43] J. H. Chow, D. E. McClelland, and M. B. Gray, "Rayleigh backscatter mitigation by RF modulation in a $100-\mathrm{km}$ remote fiber sensing system," in Sensors, and Command, Control, Communications, and Intelligence (C3I) Technologies for Homeland Security and Homeland Defense VI, E. M. Carapezza, Ed., vol. 6538, International Society for Optics and Photonics. SPIE, 2007, pp. $371-378$.

[44] Q. Guo and A. V. Tran, "Mitigation of rayleigh noise and dispersion in ream-based wdm-pon using spectrum-shaping codes," Opt. Express, vol. 20, no. 26, pp. B452-B461, Dec 2012. [Online]. Available: http://www.opticsexpress.org/abstract.cfm?URI=oe-20-26-B452

[45] G. Talli, C. W. Chow, E. K. MacHale, and P. D. Townsend, "Rayleigh noise mitigation in long-reach hybrid dwdm-tdm pons," J. Opt. Netw., vol. 6, no. 6, pp. 765-776, Jun 2007.

[46] C. H. Wang, C. W. Chow, C. H. Yeh, C. L. Wu, S. Chi, and C. Lin, "Rayleigh noise mitigation using single-sideband modulation generated by a dual-parallel mzm for carrier distributed pon," IEEE Photonics Technology Letters, vol. 22, no. 11, pp. 820-822, June 2010.

[47] Y. Rao, Z. Wang, H. Wu, Z. Ran, and B. Han, "Recent advances in phasesensitive optical time domain reflectometry (-otdr)," Photonic Sensors, vol. 11, no. 1, pp. 1-30, Mar 2021.

[48] W. Eickhoff and R. Ulrich, "Statistics of backscattering in singlemode fiber," in Integrated Optics and Optical Fiber Communication. Optical Society of America, 1981, p. TUK4. [Online]. Available: http://www.osapublishing.org/abstract.cfm?URI=OFC-1981-TUK4

[49] R. K. Staubli and P. Gysel, "Statistical properties of single-mode fiber rayleigh backscattered intensity and resulting detector current," IEEE Transactions on Communications, vol. 40, no. 6, pp. 1091-1097, June 1992.

[50] D. S. Ornstein and B. Weiss, "Statistical properties of chaotic systems," Bulletin (New Series) of the American Mathematical Society, vol. 24, no. 1, pp. 11 - 116, 1991. [Online]. Available: https://doi.org/

[51] A. Papoulis and U. Pillai, Probability, Random Variables, and Stochastic Processes, 4th ed. McGraw Hill, 2002.

[52] L. M. Berliner, "Statistics, Probability and Chaos," Statistical Science, vol. 7, no. 1 , pp. $69-90,1992$. 
[53] P. Walters, An Introduction to Ergodic Theory. Springer, 1982.

[54] D. Derickson, Fiber Optic Test and Measurement. Prentice-Hall, 1987.

[55] L. Rayleigh, "XXXIV. On the transmission of light through an atmosphere containing small particles in suspension, and on the origin of the blue of the sky," apr 1899. [Online]. Available: https://doi.org/10.1080/14786449908621276

[56] H. C. van de Hulst, Light Scattering by Small Particles. Dover Publications, 1981.

[57] Y. Onodera, S. Kohara, P. S. Salmon, A. Hirata, N. Nishiyama, S. Kitani, A. Zeidler, M. Shiga, A. Masuno, H. Inoue, S. Tahara, A. Polidori, H. E. Fischer, T. Mori, S. Kojima, H. Kawaji, A. I. Kolesnikov, M. B. Stone, M. G. Tucker, M. T. McDonnell, A. C. Hannon, Y. Hiraoka, I. Obayashi, T. Nakamura, J. Akola, Y. Fujii, K. Ohara, T. Taniguchi, and O. Sakata, "Structure and properties of densified silica glass: characterizing the order within disorder," NPG Asia Materials, vol. 12, no. 1, p. 85, Dec 2020. [Online]. Available: https://doi.org/10.1038/s41427-020-00262-z

[58] J. Schroeder, W. Wu, J. L. Apkarian, M. Lee, L.-G. Hwa, and C. T. Moynihan, "Raman scattering and boson peaks in glasses: temperature and pressure effects," Journal of Non-Crystalline Solids, vol. 349, pp. 88-97, 2004, glass Science for High Technology. 16th University Conference on Glass Science. [Online]. Available: https://www.sciencedirect.com/science/article/pii/S0022309304008002

[59] M. Kerker, The Scattering of Light and other Electromagnetic Radiation. Academic Press, 1969, vol. 16.

[60] F. Just, R. Spittel, J. Bierlich, S. Grimm, M. Jäger, and $\mathrm{H}$. Bartelt, "The influence of the fiber drawing process on intrinsic stress and the resulting birefringence optimization of pm fibers," Optical Materials, vol. 42, pp. 345-350, 2015. [Online]. Available: https://www.sciencedirect.com/science/article/pii/S0925346715000531

[61] A. D. Yablon, "Optical and mechanical effects of frozen-in stresses and strains in optical fibers," IEEE Journal of Selected Topics in Quantum Electronics, vol. 10, no. 2, pp. 300-311, March 2004.

[62] H. Jenkins and M. Nagurka, "Design and control of capstan-pulley draw system for optical fiber manufacturing," in Conference on Engineering Systems Design and Analysis, 2008, pp. 1-8. 
[63] J. P. von der Weid, R. Passy, G. Mussi, and N. Gisin, "On the characterization of optical fiber network components with optical frequency domain reflectometry," Journal of Lightwave Technology, vol. 15, no. 7, pp. 11311141, July 1997.

[64] P. J. Urban, G. C. Amaral, and J. P. von der Weid, "Fiber monitoring using a sub-carrier band in a sub-carrier multiplexed radio-over-fiber transmission system for applications in analog mobile fronthaul," Journal of Lightwave Technology, vol. 34, no. 13, pp. 3118-3125, July 2016.

[65] Y.-J. Kim, U.-C. Paek, and B. H. Lee, "Measurement of refractive-index variation with temperature by use of long-period fiber gratings," Opt. Lett., vol. 27, no. 15, pp. 1297-1299, Aug 2002.

[66] P. Billingsley, Ergodic Theory and Information. John Wiley and Sons, 1965.

[67] G.B. Xavier and T.R. da Silva and G.P. Temporão and J.P. von der Weid, "Polarisation drift compensation in $8 \mathrm{~km}$-long mach-zehnder fibre-optical interferometer for quantum communication," Electronics Letters, vol. 47, pp. 608-609(1), May 2011.

[68] E. Gómez-Déniz and L. Gómez-Déniz, "A generalisation of the rayleigh distribution with applications in wireless fading channels," Wireless Communications and Mobile Computing, vol. 13, no. 1, pp. 85-94, 2013. [Online]. Available: https://onlinelibrary.wiley.com/doi/abs/10.1002/wcm. 1097

[69] P. Beckmann, "Rayleigh Distribution and Its Generalizations," J. Res. Bur. Stand., Sec. D: Radio Sci, vol. 68D, no. 9, p. 927, 1964.

[70] M. M. Soddoqio, "Statistical Interference for Rayleigh Distributions," J. Res. Bur. Stand., Sec. D: Radio Sci, vol. 68D, no. 9, p. 1005, 1964.

[71] D. V. Churkin, S. Sugavanam, I. D. Vatnik, Z. Wang, E. V. Podivilov, S. A. Babin, Y. Rao, and S. K. Turitsyn, "Recent advances in fundamentals and applications of random fiber lasers," Adv. Opt. Photon., vol. 7, no. 3, pp. 516-569, Sep 2015.

[72] Z. Wang, H. Wu, M. Fan, Y. Rao, X. Jia, and W. Zhang, "Thirdorder random lasing via raman gain and rayleigh feedback within a half-open cavity," Opt. Express, vol. 21, no. 17, pp. 20090-20095, Aug 2013. [Online]. Available: http://www.opticsexpress.org/abstract.cfm? $\mathrm{URI}=\mathrm{oe}-21-17-20090$ 
[73] H. Wu, Z. Wang, X. Jia, P. Li, M. Fan, Y. Li, and Y. Zhu, "Flat amplitude multiwavelength brillouin-raman random fiber laser with a half-open cavity," Applied Physics B, vol. 112, no. 4, pp. 467-471, Sep 2013. [Online]. Available: https://doi.org/10.1007/s00340-013-5569-0

[74] V. S. Letokhov, "Stimulated Emission of an Ensemble of Scattering Particles with Negative Absorption," Soviet Journal of Experimental and Theoretical Physics Letters, vol. 5, p. 212, apr 1967.

[75] —-, "Generation of Light by a Scattering Medium with Negative Resonance Absorption," Soviet Journal of Experimental and Theoretical Physics, vol. 26, p. 835, apr 1968.

[76] J. Andreasen, A. A. Asatryan, L. C. Botten, M. A. Byrne, H. Cao, L. Ge, L. Labonté, P. Sebbah, A. D. Stone, H. E. Türeci, and C. Vanneste, "Modes of random lasers," Adv. Opt. Photon., vol. 3, no. 1, pp. 88-127, Mar 2011. [Online]. Available: http://aop.osa.org/abstract.cfm?URI=aop-3-1-88

[77] H. Cao, Y. G. Zhao, H. C. Ong, S. T. Ho, J. Y. Dai, J. Y. $\mathrm{Wu}$, and R. P. H. Chang, "Ultraviolet lasing in resonators formed by scattering in semiconductor polycrystalline films," Applied Physics Letters, vol. 73, no. 25, pp. 3656-3658, 1998. [Online]. Available: https://doi.org/10.1063/1.122853

[78] H. Cao, Y. G. Zhao, S. T. Ho, E. W. Seelig, Q. H. Wang, and R. P. H. Chang, "Random laser action in semiconductor powder," Phys. Rev. Lett., vol. 82, pp. 2278-2281, Mar 1999. [Online]. Available: https://link.aps.org/doi/10.1103/PhysRevLett.82.2278

[79] S. Mujumdar, V. Türck, R. Torre, and D. S. Wiersma, "Chaotic behavior of a random laser with static disorder," , vol. 76, no. 3, p. 033807, Sep. 2007.

[80] H. Cao, "Lasing in random media," Waves in Random Media, vol. 13, no. 3, pp. R1-R39, 2003. [Online]. Available: https://doi.org/10.1088/0959-7174/13/3/201

[81] _-, "Review on latest developments in random lasers with coherent feedback," Journal of Physics A: Mathematical and General, vol. 38, no. 49, pp. 10497-10535, nov 2005. [Online]. Available: https://doi.org/10.1088/0305-4470/38/49/004

[82] F. Luan, B. Gu, A. S. Gomes, K.-T. Yong, S. Wen, and P. N. Prasad, "Lasing in nanocomposite random media," Nano 
Today, vol. 10, no. 2, pp. 168-192, 2015. [Online]. Available: https://www.sciencedirect.com/science/article/pii/S1748013215000262

[83] R. Sapienza, "Determining random lasing action," Nature Reviews Physics, vol. 1, no. 11, pp. 690-695, Nov 2019. [Online]. Available: https://doi.org/10.1038/s42254-019-0113-8

[84] Z. Hu, H. Zheng, L. Wang, X. Tian, T. Wang, Q. Zhang, G. Zou, Y. Chen, and Q. Zhang, "Random fiber laser of poss solution-filled hollow optical fiber by end pumping," Optics Communications, vol. 285, no. 19, pp. 3967-3970, 2012. [Online]. Available: https://www.sciencedirect.com/science/article/pii/S0030401812004804

[85] W. Z. W. Ismail, C. Hurot, and J. M. Dawes, "Properties of random lasers in a hollow core photonic crystal fiber," Laser Physics, vol. 30, no. 3, p. 035002 , feb 2020. [Online]. Available: https://doi.org/10.1088/1555-6611/ab65c1

[86] H. Zhang, P. Zhou, H. Xiao, and X. Xu, "Efficient raman fiber laser based on random rayleigh distributed feedback with record high power," Laser Physics Letters, vol. 11, no. 7, p. 075104, may 2014. [Online]. Available: https://doi.org/10.1088/1612-2011/11/7/075104

[87] M. Pang, X. Bao, and L. Chen, "Observation of narrow linewidth spikes in the coherent brillouin random fiber laser," Opt. Lett., vol. 38, no. 11, pp. 1866-1868, Jun 2013. [Online]. Available: http://ol.osa.org/abstract.cfm?URI=ol-38-11-1866

[88] M. Pang, S. Xie, X. Bao, D.-P. Zhou, Y. Lu, and L. Chen, "Rayleigh scattering-assisted narrow linewidth brillouin lasing in cascaded fiber," Opt. Lett., vol. 37, no. 15, pp. 3129-3131, Aug 2012. [Online]. Available: http://ol.osa.org/abstract.cfm?URI=ol-37-15-3129

[89] A. A. Fotiadi, I. Lobach, and P. Mégret, "Dynamics of ultra-long Brillouin fiber laser," in Fiber Lasers X: Technology, Systems, and Applications, S. T. Hendow, Ed., vol. 8601, International Society for Optics and Photonics. SPIE, 2013, pp. 154 - 162. [Online]. Available: https://doi.org/10.1117/12.2003186

[90] D. Xiang, P. Lu, Y. Xu, L. Chen, and X. Bao, "Random brillouin fiber laser for tunable ultra-narrow linewidth microwave generation," Opt. Lett., vol. 41, no. 20, pp. 4839-4842, Oct 2016. [Online]. Available: http://ol.osa.org/abstract.cfm?URI=ol-41-20-4839 
[91] S. Popov, O. Butov, Y. Chamorovski, V. Isaev, P. Mégret, D. Korobko, I. Zolotovskii, and A. Fotiadi, "Narrow linewidth short cavity brillouin random laser based on bragg grating array fiber and dynamical population inversion gratings," Results in Physics, vol. 9, pp. 806-808, 2018. [Online]. Available: https://www.sciencedirect.com/science/article/pii/S2211379717325512

[92] J. Song, X. Dong, Z. Guo, B. Xu, and D. Wang, "Random laser with erbiumdoped fiber and fs-laser introduced random fiber grating," in 2018 Asia Communications and Photonics Conference (ACP), Oct 2018, pp. 1-3.

[93] L. Wang, X. Dong, P. P. Shum, and H. Su, "Tunable erbium-doped fiber laser based on random distributed feedback," IEEE Photonics Journal, vol. 6, no. 5, pp. 1-5, Oct 2014.

[94] W. L. Zhang, S. W. Li, R. Ma, Y. J. Rao, Y. Y. Zhu, Z. N. Wang, X. H. Jia, and J. Li, "Random distributed feedback fiber laser based on combination of er-doped fiber and single-mode fiber," IEEE Journal of Selected Topics in Quantum Electronics, vol. 21, no. 1, pp. 44-49, Jan 2015.

[95] T. Zhu, X. Bao, and L. Chen, "A self-gain random distributed feedback fiber laser based on stimulated rayleigh scattering," Optics Communications, vol. 285, no. 6, pp. 1371-1374, 2012. [Online]. Available: https://www.sciencedirect.com/science/article/pii/S0030401811013125

[96] Y. Xu, "Fiber random grating and its applications," Ph.D. dissertation, University of Ottawa, 2017. [Online]. Available: https://ruor.uottawa.ca/ bitstream/10393/36597/3/Xu_Yanping_2017_thesis.pdf

[97] H. A. Shawki, H. E. Kotb, and D. Khalil, "Narrow line width semiconductor optical amplifier based random laser," in Fiber Lasers XIV: Technology and Systems, C. A. Robin and I. Hartl, Eds., vol. 10083, International Society for Optics and Photonics. SPIE, 2017, pp. 431 - 436. [Online]. Available: https://doi.org/10.1117/12.2251786

[98] H. Shawki, H. Kotb, and D. Khalil, "Single-longitudinal-mode broadband tunable random laser," Opt. Lett., vol. 42, no. 16, pp. 3247-3250, Aug 2017. [Online]. Available: http://ol.osa.org/abstract.cfm?URI=ol-42-16-3247

[99] H. A. Shawki, H. E. Kotb, and D. Khalil, "Narrow line width dual wavelength semiconductor optical amplifier based random fiber laser," in Fiber Lasers XV: Technology and Systems, I. Hartl and A. L. Carter, Eds., vol. 10512, International Society for Optics and Photonics. SPIE, 2018, pp. 416 - 422. [Online]. Available: https://doi.org/10.1117/12.2289189 
[100] T. Zhu, X. Bao, L. Chen, H. Liang, and Y. Dong, "Experimental study on stimulated rayleigh scattering in optical fibers," Opt. Express, vol. 18, no. 22, pp. 22958-22963, Oct 2010. [Online]. Available: http://www.opticsexpress.org/abstract.cfm?URI=oe-18-22-22958

[101] B. Redding, M. A. Choma, and H. Cao, "Speckle-free laser imaging using random laser illumination," Nature Photonics, vol. 6, no. 6, pp. 355-359, Jun 2012. [Online]. Available: https://doi.org/10.1038/nphoton.2012.90

[102] B. Saxena, X. Bao, and L. Chen, "Suppression of thermal frequency noise in erbium-doped fiber random lasers," Opt. Lett., vol. 39, no. 4, pp. 1038-1041, Feb 2014. [Online]. Available: http:/ /ol.osa.org/abstract.cfm?URI=ol-39-4-1038

[103] Z. N. Wang, Y. J. Rao, H. Wu, P. Y. Li, Y. Jiang, X. H. Jia, and W. L. Zhang, "Long-distance fiber-optic pointsensing systems based on random fiber lasers," Opt. Express, vol. 20, no. 16, pp. 17695-17700, Jul 2012. [Online]. Available: http://www.opticsexpress.org/abstract.cfm?URI=oe-20-16-17695

[104] X.-H. Jia, Y.-J. Rao, Z.-N. Wang, W.-L. Zhang, Y. Jiang, J.-M. Zhu, and Z.-X. Yang, "Towards fully distributed amplification and high-performance long-range distributed sensing based on random fiber laser," in OFS2012 22nd International Conference on Optical Fiber Sensors, vol. 8421, International Society for Optics and Photonics. SPIE, 2012, pp. 410 413. [Online]. Available: https://doi.org/10.1117/12.975168

[105] J. c. v. Mináŕ, H. de Riedmatten, C. Simon, H. Zbinden, and N. Gisin, "Phase-noise measurements in long-fiber interferometers for quantumrepeater applications," Phys. Rev. A, vol. 77, p. 052325, May 2008. [Online]. Available: https://link.aps.org/doi/10.1103/PhysRevA.77.052325

[106] D. Sharma, H. Ramachandran, and N. Kumar, "Lévy statistics of emission from a novel random amplifying medium: an optical realization of the arrhenius cascade," Opt. Lett., vol. 31, no. 12, pp. 1806-1808, Jun 2006. [Online]. Available: http://ol.osa.org/abstract.cfm?URI=ol-31-12-1806

[107] S. Lepri, S. Cavalieri, G.-L. Oppo, and D. S. Wiersma, "Statistical regimes of random laser fluctuations," Phys. Rev. A, vol. 75, p. 063820, Jun 2007. [Online]. Available: https://link.aps.org/doi/10.1103/PhysRevA.75.063820 
[108] L. Angelani, C. Conti, G. Ruocco, and F. Zamponi, "Glassy behavior of light," Phys. Rev. Lett., vol. 96, p. 065702, Feb 2006. [Online]. Available: https://link.aps.org/doi/10.1103/PhysRevLett.96.065702

[109] —, "Glassy behavior of light in random lasers," Phys. Rev. B, vol. 74, p. 104207, Sep 2006. [Online]. Available: https://link.aps.org/doi/10.1103/PhysRevB.74.104207

[110] N. Ghofraniha, I. Viola, F. Di Maria, G. Barbarella, G. Gigli, L. Leuzzi, and C. Conti, "Experimental evidence of replica symmetry breaking in random lasers," Nature Communications, vol. 6, no. 1, p. 6058, Jan 2015. [Online]. Available: https://doi.org/10.1038/ncomms7058

[111] A. S. L. Gomes, B. C. Lima, P. I. R. Pincheira, A. L. Moura, M. Gagné, E. P. Raposo, C. B. de Araújo, and R. Kashyap, "Glassy behavior in a one-dimensional continuous-wave erbium-doped random fiber laser," Phys. Rev. A, vol. 94, p. 011801, Jul 2016. [Online]. Available: https://link.aps.org/doi/10.1103/PhysRevA.94.011801

[112] R. W. Boyd, K. Rza ewski, and P. Narum, "Noise initiation of stimulated brillouin scattering," Phys. Rev. A, vol. 42, pp. 5514-5521, Nov 1990. [Online]. Available: https://link.aps.org/doi/10.1103/PhysRevA.42.5514

[113] W. Jinsong, T. Weizhong, and Z. Wen, "Stimulated brillouin scattering initiated by thermally excited acoustic waves in absorption media," Optics Communications, vol. 123, no. 4, pp. 574-576, 1996. [Online]. Available: https://www.sciencedirect.com/science/article/pii/0030401895005455

[114] M. Nikles, L. Thevenaz, and P. Robert, "Brillouin gain spectrum characterization in single-mode optical fibers," Journal of Lightwave Technology, vol. 15 , no. 10 , pp. 1842-1851, 1997.

[115] V. Milner and A. Z. Genack, "Photon Localization Laser: Low-Threshold Lasing in a Random Amplifying Layered Medium via Wave Localization," Phys. Rev. Lett., vol. 94, p. 073901, Feb 2005. [Online]. Available: https://link.aps.org/doi/10.1103/PhysRevLett.94.073901

[116] M. Gagné and R. Kashyap, "Demonstration of a $3 \mathrm{mw}$ threshold er-doped random fiber laser based on a unique fiber bragg grating," Opt. Express, vol. 17, no. 21, pp. $19067-19074$, Oct 2009. [Online]. Available: http://www.opticsexpress.org/abstract.cfm?URI=oe-17-21-19067

[117] S. A. Babin, D. V. Churkin, S. I. Kablukov, M. A. Rybakov, and A. A. Vlasov, "All-fiber widely tunable raman fiber laser with controlled output 
spectrum," Opt. Express, vol. 15, no. 13, pp. 8438-8443, Jun 2007. [Online]. Available: http://www.opticsexpress.org/abstract.cfm?URI=oe-15-13-8438

[118] E. Belanger, M. Bernier, D. Faucher, D. Cote, and R. Vallee, "High-power and widely tunable all-fiber raman laser," Journal of Lightwave Technology, vol. 26, no. 12, pp. 1696-1701, 2008.

[119] Y.-E. Im, S. Hann, H. Kim, D.-H. Kim, and C.-S. Park, "An all-fibre robust and tunable raman fibre laser with reconfigurable asymmetric cavities," Measurement Science and Technology, vol. 20, no. 3, p. 034022, feb 2009. [Online]. Available: https://doi.org/10.1088/0957-0233/20/3/034022

[120] M. Bravo, M. Fernandez-Vallejo, and M. Lopez-Amo, "Internal modulation of a random fiber laser," Opt. Lett., vol. 38, no. 9, pp. 1542-1544, May 2013. [Online]. Available: http://ol.osa.org/abstract.cfm?URI=ol-38-9-1542

[121] W. Margulis, A. Das, J. P. von der Weid, and A. S. L. Gomes, "Hybrid electronically addressable random fiber laser," Opt. Express, vol. 28, no. 16, pp. 23388-23396, Aug 2020. [Online]. Available: http://www.opticsexpress.org/abstract.cfm?URI=oe-28-16-23388

[122] MPZ-LN-40 - Lithium Niobate high-bandwidth electro-optic phase modulator, iXblue Photonics., https://photonics.ixblue.com/store/ lithium-niobate-electro-optic-modulator/phase-modulators.

[123] PZ2-SM2 - High-efficiency Fiber Stretcher, Optiphase - Haliburton., http: //www.optiphase.com/data_sheets/PZ2_Data_Sheet_Rev_G.pdf. 